\title{
Post-azacitidine clone size predicts long-term clinical outcome of patients with myelodysplastic syndromes and related myeloid neoplasms
}

Seishi Ogawa ( $\nabla$ sogawa-tky@umin.ac.jp )

Kyoto University

Magnus Tobiasson

Karolinska Institute

Shinya Sato

Nagasaki University

Elsa Bernard

Memorial Sloan Kettering Cancer Center

Shigeki Ohtake

Kanazawa University

June Takeda

Graduate School of Medicine, Kyoto University

Maria Creignou

Karolinska Institute

Lanying Zhao

Kyoto University

Manabu Kusakabe

University of Tsukuba

Yuhei Shibata

Gifu Municipal Hospital

Nobuhiko Nakamura

Gifu University Hospital

Mizuki Watanabe

Kyoto University

Nobuhiro Hiramoto

Kobe City Medical Center General Hospital

Yusuke Shiozawa

Graduate School of Medicine, Kyoto University

Yuichi Shiraishi

National Cancer Center Research Institute

Hiroko Tanaka 
University of Tokyo https://orcid.org/0000-0001-9634-8922

\section{Kenichi Yoshida}

Kyoto University https://orcid.org/0000-0003-4612-2778

\section{Nobuyuki Kakiuchi}

Kyoto University https://orcid.org/0000-0003-4893-5414

\section{Hideki Makishima}

https://orcid.org/0000-0001-5983-8578

\section{Masahiro Nakagawa}

kyoto Univeristy

\section{Kensuke Usuki}

NTT Kanto Medical Center

\section{Mitsumasa Watanabe}

Hyogo Prefectural Amagasaki General Medical Center

\section{Kazunori Imada}

Japan Red Cross Osaka Hospital

\section{Hiroshi Handa}

Gunma University

\section{Masataka Taguchi}

Sasebo City General Hospital https://orcid.org/0000-0002-0642-5814

\section{Toru Kiguchi}

Chugoku Central Hospital

\section{Kazuma Ohyashiki}

Tokyo Medical University

\section{Takayuki Ishikawa}

Kobe City Medical Center General Hospital

\section{Akifumi Takaori-Kondo}

Department of Hematology and Oncology, Graduate School of Medicine, Kyoto University https://orcid.org/0000-0001-7678-4284

\section{Hisashi Tsurumi}

Gifu University

\section{Senji Kasahara}

Gifu Municipal Hospital

\section{Shigeru Chiba}

University of Tsukuba

\section{Tomoki Naoe}

Nagoya Medical Center

\section{Satoru Miyano}

The University of Tokyo, Institute of Medical Science

\section{Elli Papaemmanuil}


Memorial Sloan Kettering Cancer Center https://orcid.org/0000-0003-1709-8983

\section{Yasushi Miyazaki}

Department of Hematology, Nagasaki University Hospital

\section{Eva Hellstrom-Lindberg}

Karolinska Institutet

Article

Keywords: myeloid neoplasms, azacitidine therapy. next-generation sequencing

Posted Date: May 18th, 2021

DOI: https://doi.org/10.21203/rs.3.rs-504223/v1

License: (c) (1) This work is licensed under a Creative Commons Attribution 4.0 International License. Read Full License 


\section{Post-azacitidine clone size predicts long-term clinical outcome of patients with myelodysplastic syndromes and related myeloid neoplasms}

Yasuhito Nannya ${ }^{* 1}$, Magnus Tobiasson ${ }^{* 2}$, Shinya Sato ${ }^{3,4}$, Elsa Bernard ${ }^{5}$, Shigeki Ohtake ${ }^{6}$, June Takeda ${ }^{1}$, Maria Creignou ${ }^{2}$, Lanying Zhao ${ }^{1}$, Manabu Kusakabe ${ }^{7}$, Yuhei Shibata ${ }^{8}$, Nobuhiko Nakamura ${ }^{9}$, Mizuki Watanabe ${ }^{10}$, Nobuhiro Hiramoto ${ }^{11}$, Yusuke Shiozawa ${ }^{1}$, Yuichi Shiraishi ${ }^{12}$, Hiroko Tanaka ${ }^{13}$, Kenichi

Yoshida ${ }^{1}$, Nobuyuki Kakiuchi ${ }^{1}$, Hideki Makishima ${ }^{1}$, Masahiro Nakagawa ${ }^{1}$, Kensuke Usuki ${ }^{14}$, Mitsumasa Watanabe ${ }^{15}$, Kazunori Imada ${ }^{16}$, Hiroshi Handa ${ }^{17}$, Masataka Taguchi ${ }^{18}$, Toru Kiguchi ${ }^{19}$, Kazuma

Ohyashiki ${ }^{20}$, Takayuki Ishikawa ${ }^{11}$, Akifumi Takaori-Kondo ${ }^{10}$, Hisashi Tsurumi ${ }^{9}$, Senji Kasahara ${ }^{8}$, Shigeru Chiba $^{7}$, Tomoki Naoe ${ }^{4,21}$, Satoru Miyano ${ }^{13}$, Elli Papaemanuil ${ }^{5}$, Yasushi Miyazaki ${ }^{3,4}$,

Eva Hellström-Lindberg ${ }^{\dagger 2}$ and Seishi Ogawa ${ }^{\dagger 1,22,23}$

*Contributed equally to the work.

$\dagger$ Shared senior authorship.

\section{Affiliations:}

${ }^{1}$ Department of Pathology and Tumor Biology, Graduate School of Medicine, Kyoto University, Kyoto, Japan

${ }^{2}$ Division of Hematology, Department of Medicine, Karolinska University Hospital, Stockholm, Sweden.

${ }^{3}$ Department of Hematology, Atomic Bomb Disease and Hibakusha Medicine Unit, Atomic Bomb Disease Institute, Nagasaki University, Nagasaki, Japan

${ }^{4} J a p a n$ Adult Leukemia Study Group, Japan

${ }^{5}$ Memorial Sloan Kettering Cancer Center, New York, NY, USA

${ }^{6}$ Kanazawa University, Kanazawa, Japan

${ }^{7}$ Department of Hematology, Tsukuba University, Tsukuba, Japan

${ }^{8}$ Department of Hematology, Gifu Municipal Hospital, Gifu, Japan

${ }^{9}$ Department of Hematology \& Infectious Disease, Gifu University Hospital, Gifu, Japan

${ }^{10}$ Department of Hematology and Oncology, Kyoto University, Kyoto, Japan

${ }^{11}$ Department of Hematology, Kobe City Medical Center General Hospital, Hyogo, Japan

${ }^{12}$ Division of Cellular Signaling, National Cancer Center Research Institute, Tokyo, Japan

${ }^{13}$ Department of Integrated Data Science, M\&D Data Science Center, Tokyo Medical and Dental University, Tokyo, Japan

${ }^{14}$ Department of Hematology, NTT Medical Center Tokyo, Tokyo, Japan

${ }^{15}$ Department of Hematology, Hyogo Prefectural Amagasaki General Medical Center, Hyogo, Japan

${ }^{16}$ Department of Hematology, Japan Red Cross Osaka Hospital, Osaka, Japan

${ }^{17}$ Department of Hematology, Gunma University, Gunma, Japan

${ }^{18}$ Department of Hematology, Sasebo City General Hospital, Nagasaki, Japan 
${ }^{19}$ Department of Hematology, Chugoku Central Hospital, Hiroshima, Japan

${ }^{20}$ Department of Hematology, Tokyo Medical University, Tokyo, Japan

${ }^{21}$ Nagoya Medical Center, Aichi, Japan

${ }^{22}$ Institute for the Advanced Study of Human Biology (WPI-ASHBi), Kyoto University, Kyoto, Japan.

${ }^{23}$ Department of Medicine, Center for Hematology and Regenerative Medicine, Karolinska Institute, Stockholm, Sweden

\section{Correspondence should be addressed to:}

Eva Hellström Lindberg (eva.hellstrom-lindberg@ki.se) or Seishi Ogawa (sogawa-tky@umin.ac.jp).

\section{Conflict of interest disclosure:}

The authors declare no conflict of interest.

Text word count: 4620 words

Number of figures: 5; Number of table:1; Number of references: 71 
2 Azacitidine is a mainstay of therapy for high-risk MDS and other myeloid neoplasms. A significant 3 correlation between azacitidine response and clinical outcome suggests a potential role of mutational 4 profiling based on next-generation sequencing (NGS) before and after therapy in evaluating response 5 and predicting overall survival (OS), which however has not fully elucidated. Here through NGS-based 6 mutational profiling of large cohorts $(n=451)$ of azacitidine-treated patients with high-risk MDS and 7 other myeloid neoplasms, we show significant roles of multi-hit TP53 and germline DDX41 mutations 8 in pre-treatment samples and post-treatment clone size in the evaluation/prediction of azacitidine 9 response and OS after azacitidine therapy, which outperformed the prediction based only on clinical response and IPSS-R score. Post-treatment clone size strongly correlated with clinical response with exception of large persistent mutations frequently affecting TET2 after achieving complete remission 12 and those with DDX41 mutations, which poorly correlated with clinical response. Our results highlight the importance of evaluating mutations in both pre- and post-treatment samples in the assessment of response and the prediction of OS after azacitidine therapy. 


\section{INTRODUCTION}

Azacitidine, together with another hypomethylating agent (HMA), decitabine, is a standard choice of therapy for patients with higher-risk myelodysplastic syndromes (MDS) and other related myeloid neoplasms, including myelodysplastic/myeloproliferative neoplasms (MDS/MPN), and oligoblastic acute myeloid leukemia (AML). It can induce a clinical remission in 50-60\% of treated patients ${ }^{1,2}$ and has been shown to prolong survival compared to standard regimens ${ }^{3}$ while others respond poorly or not at all and a major clinical question is who best benefits from azacitidine treatment. In fact, many studies have investigated biomarkers that can predict initial response and long-term survival following azacitidine therapy, particularly in terms of gene mutations ${ }^{4-27}$. However, despite many efforts, no reliable biomarkers have been identified and results have been inconsistent across studies. Moreover, small numbers of enrolled patients and variable study designs with respect to patient enrollment criteria, definition of outcomes, genotyped genes, and explanatory factors tested do not allow for a direct comparison between or prevented a successful meta-analysis of these results ${ }^{22}$.

Another problem that complicates the identification of such biomarkers is the lack of reliable measures for the evaluation of the initial response to therapy. To date the IWG criteria have been widely used for the assessment of therapeutic response in MDS and related disorders, in which blast count and hematological improvement are evaluated. Although highly correlated with tumor clearance in $\mathrm{AML}$, blast count may not correctly reflect tumor burden in $\mathrm{MDS}^{28}$, apart from an inter-observer variance in blast cell quantification ${ }^{29}$. In addition, blood cell counts could be influenced by the use of cytotoxic agents, particularly when they are assessed before BM fully recovers between treatment cycles $^{30}$. In this regard, next-generation sequencing (NGS) might provide a more robust platform to measure a molecular response or a reduction in tumor burden in terms of a variant allele frequency, or clone size, of somatic mutations detected before and after therapy. In fact, several studies evaluated a molecular response in patients who were treated with hypomethylating agents using NGS ${ }^{15,31-35}$. However, the size of each study was relatively small to fully evaluate the impact of tumor burden on clinical response and long-term outcomes, although several studies have reported a correlation between a reduced tumor burden and clinical response, particularly in TP53-mutated AML and $\operatorname{MDS}^{14,31,32}$.

Here, we investigated the effects of gene mutations on clinical outcomes in the largest cohort of azacitidine-treated patients with MDS, MDS/MPN, and oligoblastic AML (blast percentage $\leq 30 \%$ at enrollment) ever studied ( $n=341$ ), where genetic alterations were comprehensively analyzed by targeted-capture sequencing of major drivers for myeloid malignancies. The major results were validated using an independent cohort of azacitidine-treated patients ( $n=110)$. In addition, mutational profiles before and after azacitidine treatment were assessed for 290 cases to explore their impact on clinical response and survival. Finally, the role of subsequent transplantation for otherwise intractable TP53-mutated cases was investigated in whom a sufficient reduction of TP53-mutant clone was obtained with azacitidine treatment.

\section{RESULTS}

Study subjects 
The discovery cohort consisted of azacitidine-treated patients from the prospective JALSG MDS-212 study $^{36}(n=178)$ and a retrospective cohort collected at Karolinska Institute and University Hospital ( $n$ = 163). The validation cohort included 110 azacitidine-treated patients who were retrospectively enrolled from 13 collaborating institutes in Japan (Supplementary Fig. 1). Demographic and clinical characteristics, including disease classification, IPSS-R risk, karyotype risk, blood count, marrow blast, of both discovery and validation cohorts are summarized in Table 1. The treatment schedule, OS, and other demographic data were largely similar between both cohorts except for an overrepresentation of oligoblastic AML (6.2\% versus $13.6 \%)$ and underrepresentation of MDS/MPN (7.3\% versus $3.6 \%)$ in the validation cohort (Extended Data Fig. 1a, 1b, Table 1). The response to azacitidine was evaluable in 311 and 85 patients in the discovery and the validation cohorts, respectively (Table 1).

\section{Genetic abnormalities in the discovery and the validation cohorts}

With a median depth of 565× (range, 81-2,501×) (Supplementary Fig. 2), targeted sequencing of the discovery cohort identified 1,069 and 730 single nucleotide variants (SNVs) and short indels in 326 preand 209 post-treatment samples, respectively. In sequencing-based copy number analysis, 770 and 352 genomic segments exhibited copy number abnormalities (CNAs) affecting in 207 pre- and 103 post-treatment samples, respectively (Supplementary Tables 2, 3). Reflecting an overrepresentation of higher risk patients with indication of azacitidine therapy, $-7 / \operatorname{del}(7 q)(n=87)$, TP53 mutation ( $n=$ 87 ), $-5 / \operatorname{del}(5 q)$ ( $n=80), R U N X 1$ mutation ( $n=73)$, loss-of-heterozygosity (LOH) of $17 p(n=72)$, and TET2 mutation ( $n=71$ ) were among the most frequent abnormalities (Supplementary Fig. 3). Based on our recent report that revealed distinct characterization and outcomes of MDS cases according to allelic state of TP53 mutation ${ }^{37}$, we analyzed cases with mono-allelic and multi-hit TP53 mutation separately. Almost an equivalent coverage (a median depth of 624x; range, 348-1,105x) was obtained in the analysis of the validation cohort, in which we called a total of 290 SNVs or indels and 110 CNAs in 108 pre-treatment samples and 131 SNVs and 98 CNAs in 47 post-treatment samples (Supplementary Tables 4,5 ). The mutation profile was largely comparable to that of the discovery cohorts, except for a substantial underrepresentation of SRSF2 and RAS pathway mutations in the validation cohort, likely reflecting a lower representation of MDS/MPN cases in the latter cohort (Supplementary Fig. 3). Based on these findings, we first interrogated the best response to azacitidine therapy.

\section{Correlations between genetic abnormalities and treatment response}

In univariate analysis, 17p LOH (23/65), multi-hit TP53 mutation (24/70), higher karyotype (KT) risk (31/112), and $-5 / \operatorname{del}(5 q)(21 / 71)$ were significantly associated with a higher complete remission (CR) rate, whereas lower hemoglobin levels (<80 g/L) (6/67) and ASXL1 mutation (4/53) predicted a lower response (Extended Data Fig. 2a). These trends were also seen in the validation cohort, although not reaching statistical significance (Extended Data Fig. 2b). A lower response in ASXL1-mutated cases has also been reported in a previous study of azacitidine-treated patients with chronic myelomonocytic leukemia ${ }^{35}$. In a multivariate analysis that included significant and sub-significant $(P<0.10)$ factors in the univariate analysis, only multi-hit TP53 mutation and lower hemoglobin remained significant, although ASXL1 mutation was still incorporated in the model with a marginal $P$-value (Methods, Fig. 1a, Extended Data Fig. 2c). We observed no significant difference in CR rate itself and other responses between mono-allelic versus multi-hit TP53 mutation (2/8 versus $24 / 70$ for CR, fisher's exact test, $P=$ 0.72) (Extended Data Fig. 2d), although this needs further evaluations due to a small number of 
patients with mono-allelic mutation $(n=8)$. In contrast to previous studies, none of the genetic abnormalities we evaluated significantly correlated with overall response (as defined by $C R$, marrow CR (mCR), or partial remission (PR) $)^{11,18}$ except for 17p LOH (Fig. 1b, Extended Data Fig. 3a, 3b). We also explored the factors predicting progressive disease (PD). Univariate analysis suggested several positive (higher karyotype risk, del(12p), lower absolute nucleated cells (ANC) and hemoglobin, 7/del(7q) and higher age) and negative (STAG2 mutation) association with PD, of which higher age, higher karyotype risk, and STAG2 mutation remained significant in multivariate analysis (Extended Data Fig. 3c, 3d).

\section{Effects of gene mutations on OS following azacitidine treatment}

We next evaluated the effect of genetic alterations on OS. In univariate analysis, multi-hit TP53 mutation, $-5 / \operatorname{del}(5 q),-7 / \operatorname{del}(7 q), 17 p \mathrm{LOH}$, del(18p), 18q LOH, and del(12p) were associated with a shorter OS, together with older age ( $>72$ years old), higher marrow blast ratio (>10 \%), higher KT risk, lower hemoglobin, and lower platelet count $\left(<50 \times 10^{9} / \mathrm{L}\right)$. By contrast, mutations in DDX41 and SRSF2 predicted a favorable outcome (Extended Data Fig. 4a), which was largely recapitulated in the validation cohort (Extended Data Fig. 4b). In line with previous reports ${ }^{38}$, responders showed a significantly longer OS compared with non-responders, with a median OS of 30.5, 22.3, 17.9, and 12.8 months in patients with $\mathrm{CR}, \mathrm{mCR} / \mathrm{PR}, \mathrm{SD}$ and $\mathrm{PD}$, respectively (two-sided log-rank test, $P<0.0001$ ) (Extended Data Fig. 4c). The prolonged OS in responders was seen even among patients with multi-hit TP53 mutations, which is notoriously associated with very poor outcome, although the prognosis was still dismal with a median survival of 17.0 months for CR cases (Extended Data Fig. 4d). Patients with mono-allelic TP53 mutations had a significantly longer OS that was comparable to that of TP53unmutated patients, compared to those with multi-hit mutations, confirming the effect of allelic state of TP53 mutations on prognosis also in the context of azacitidine treatment ${ }^{37}$ (two-sided log-rank test, $P=0.034$ ) (Fig. 1C). Of note, multi-hit TP53 mutation further stratified patients in the IPSS-R very highrisk category (two-sided log-rank test, $P=0.02$ ) (Extended Data Fig. 4e). In accordance with previous reports for azacitidine and decitabine ${ }^{9,16,39-41}$, the higher CR rate in TP53-mutated patients was not necessarily translated into favorable long-term outcomes. This is explained by a shorter progression free survival in TP53-mutated patients compared with that in TP53-unmutated patients (8.4 versus 36.3 months, two-sided log-rank test, $P<0.0001$ ) (Extended Data Fig. 4f).

DDX41 is one of the most frequent targets of germline mutations responsible for the development of AML and MDS, accounting for 2.4-3.4\% of the cases ${ }^{42-44}$. Most DDX41-mutated cases have a pathogenic likely-germline variant, of whom $50-79 \%$ are accompanied by a somatic mutation, typically the $\mathrm{R} 525 \mathrm{H}$ variant ${ }^{42-44}$. In total, we found 31 cases with $D D X 41$ mutations in the discovery cohort, including eight with germline mutation alone, 22 with both germline and somatic mutations, and one with somatic mutations alone (Supplementary Table 6). DDX41-mutated cases had a significantly longer OS compared with DDX41-unmutated cases irrespective of the mutation state (two-sided log-rank test, $P=0.002$ for unmutated versus germline mutations; $P=0.001$ for unmutated versus germline plus somatic mutations, Extended Data Fig. $\mathbf{4 g}$ ) or IPSS-R risks (two-sided log-rank test, $P=0.04$ for IPSS-R very low/low/intermediate, $P=0.02$ for IPSS-R high, $P=0.03$ for IPSS-R very high-risk group) (Extended Data Fig. 4h). Nevertheless, there was no difference in response profile between DDX41-mutated and unmutated cases (Extended Data Fig. 4i). 
In multivariate analysis, best response (achieved overall response or not), higher age, lower hemoglobin level, lower platelet count, higher blast\%, higher KT risk, multi-hit TP53 mutations, and DDX41 mutation were independently associated with OS (Extended Data Fig. 5a, 5b). Most of the significant variables overlapped with the factors incorporated into the IPSS-R. Indeed IPSS-R score significantly correlated with OS in our cohort (Extended Data Fig. 5c), as repeatedly shown in previous reports ${ }^{14,45,46}$. Thus, we re-constructed a multivariate model by replacing these variables with IPSS-R score and obtained a final model consisting of four independent, significant variables, a multi-hit TP53 lesion, DDX41 mutation, best response, as well as IPSS-R (Fig 1d). Based on the model, we generated a new scoring system, by which patients were stratified into three risk groups, LOW, INT, and HIGH, with a median survival of 32 (confidential interval (Cl): 25-43), 16 (Cl: 15-18), and 10 (Cl: 8-13) months, respectively (Fig. 1e). The model significantly improved compared with an IPSS-R alone model as shown by a higher c-index for the new scoring system (Extended Data Fig. $\mathbf{5 d}$ ). In fact, each IPSS-R group was further stratified into two subgroups with different OS (Extended Data Fig. 5e). These results were confirmed in the validation cohort (Extended Data Fig. 5f).

Change in clone size during azacitidine therapy

Given that our sequencing panel encompassed most of the driver genes implicated in MDS, we were able to correctly measure the size of both founder clones and subclones before and after azacitidine in most, if not all, cases, which might be used to better evaluated therapeutic response on the basis of the post-treatment reduction of tumor burden. Post-treatment samples were obtained from 290 (64\%) cases from the combined discovery and validation cohort after a median of four treatment cycles (Extended Data Fig. 1c). In total, we detected 980 driver mutations among samples from 268 cases, of which 851 and 783 were found in 260 pre- and 234 post-azacitidine samples, respectively, with 654 being shared in both samples. Mutations were classified into major clone (MC) mutations when they were contained in the major clone within each of pre- or post-azacitidine samples; otherwise, they were thought to represent non-MC (Fig. 2a, Methods). Thus, 448 of the 851 mutations represented $M C$ in pre-treatment samples (MCre) (Fig. 2a left panel). Among these, 387 also represented the largest clone in post-azacitidine samples (MC) (Fig. 2a right panel, Methods). MCs were most frequently explained by TP53 mutations $(n=68)$, followed by those in TET2 $(n=39), \operatorname{SRSF2}(n=36)$, RUNX1 ( $n=25)$, and DDX41 ( $n=19)$. By contrast, on the mutated gene basis, DDX41 (95\%), U2AF1 (82\%), TP53 (81\%), and DNMT3A (71\%) more frequently represented MC mutations, compared to ASXL1 (31\%), RUNX1 (32\%), and STAG2 (33\%) (Fig. 2b). Changes in clone size in pre- and post-treatment samples are shown in Supplementary Fig. 4.

We next correlated the response to azacitidine with the size of tumor component before and after azacitidine treatment that was estimated by the average size of $\mathrm{MC}$ mutations ( ${ }^{\text {ave }} \mathrm{MC}^{\text {pre }}$ and ${ }^{\text {ave }} \mathrm{MC}^{\text {post }}$, respectively )(Fig. 2a). We found that ${ }^{\text {ave }} \mathrm{MC}^{\text {post }}$ showed a strong correlation with response (Jonckheere-Terpstra test, $P=0.0001$ ) (Fig. $2 \mathrm{c}$ ), while an only modest correlation was noted between ${ }^{\text {ave }} \mathrm{MC}^{\text {pre }}$ and response (Jonckheere-Terpstra test, $P=0.04$ ) (Extended Data Fig. 6a). Actually, in the 48 cases who achieved $\mathrm{CR}$, the median value of ${ }^{\text {ave }} \mathrm{MC}^{\text {post }}$ was as small as 0.066 including 22 cases that achieved a complete disappearance of the MCs after treatment. Nevertheless, 7 cases showed large clones with $>0.40$ ave $\mathrm{MC}^{\text {post }}$ even after achieving $\mathrm{CR}$, of which 4 had subclones that shrank after treatment (Fig. 2c, cases \#1 to \#4, Fig. 2d). MC mutations that persisted in these cases after achieving $180 \mathrm{CR}$ were represented by TET2 $(\mathrm{N}=3)$ and DNMT3A $(\mathrm{N}=1)$ mutations, both of which are the most 
frequently mutated genes in clonal hematopoiesis $(\mathrm{CH})^{47-49}$. Thus, with a reduced blast count and normalization of blood counts, these cases may represent reversion to $\mathrm{CH}$.

A total of 131 driver mutations were detected in either pre- or post-treatment samples in the 48 CR cases (Fig. 2e), in which a distinct pattern of clonal changes was observed according to driver mutations. As expected from a high CR rate of TP53-mutated cases, TP53 was the most frequently mutated gene in CR cases, accounting for 27 mutations in 23 cases. TP53-mutated clones were characterized by high percentage to be classified as MCs (24 of 27, 88.9\%) and marked reduction in clone size in post-treatment samples (Fig. 2f). By contrast, out of 20 TET-mutated clones in 11 CR cases, only 8 were classified as MC, where large TET2-mutant clones persisted after azacitidine treatment. Of interest, all RUNX1-mutated clones $(\mathrm{n}=11$ ) detected in 8 pre-treatment samples showed a reduced clone size after treatment (Supplementary Fig. 5). However, 8 of these 11 RUNX1 mutations represented subclones most commonly of TP53-mutated MC and the apparent sensitivity of RUNX1 mutants might be explained by co-occurring TP53 mutations. Almost always present in a subclone, NPM1 and KRAS mutations in MDS patients have been implicated in progression to secondary $A M L^{50-}$

52. In line with this, both mutations in our study disappeared when CR was achieved, which has also been reported in a recent study ${ }^{53}$. Likewise, a reduce size of $E Z H 2$-mutated clones also correlated with a favorable clinical response (Fig. 2f).

\section{Effect of DDX41 mutations on azacitidine therapy}

In accordance with a strong correlation between response and ${ }^{\text {ave }} \mathrm{MC}^{\text {post }}$, patients who failed to achieve response had a large ave $\mathrm{MC}^{\text {post; }}$; the median values of ave ${ }^{\mathrm{MC} \mathrm{C}^{\text {post }}}$ among 158 cases with SD+HI, SD-HI, and PD were 0.65, 0.80, and 0.82, respectively (Fig. 2c). Among these, however, 10 showed a small ave MC $^{\text {post }}$ $(<0.10)$ (Fig. 2c, cases \#5 to \#14). Intriguingly, these cases were highly enriched for germline DDX41 mutations, which accounted for 8 out of the 10 cases (Fisher's exact test, $P<0.0001$ ) (Fig. 3a, PD cases; Fig. 3b, SD case; Extended Data Fig. 6b). Among these 8 cases, only 2 acquired driver mutations in the post-treatment samples, including a novel somatic DDX41 mutation in both cases (Extended Data Fig. 6b, cases \#6, \#9), while the remaining 6 cases did not acquire novel driver mutations or show an expansion of pre-existing mutant clones. To explore possible mutations in other drivers that were not included in our target panel, we performed whole genome sequencing (WGS) in 4 of these 6 cases. Although a clonal population was suggested from the VAF distribution of somatic mutations, no known driver mutations were identified that should have conferred a survival advantage to these clones. Therefore, the poor response in these cases remains unexplained (Extended Data Fig. 7, Supplementary Data Table 7).

In the entire paired cohort, somatic DDX41 mutations were observed in 22 cases, all of which accompanied a germline DDX41 mutation. These cases with somatic DDX41 mutations had distinct features. First, while somatic DDX41 mutations represented the major clone in most (95\%) cases (Fig. $\mathbf{2 b )}$, their clone size was significantly smaller than expected for a major clone in other cases having MC mutations other than DDX41 mutations (Fig. 3c, t-test for clone size of mutations in DDX41 and the other genes, $P<0.0001)$. This is intriguing, given that $D D X 41$-mutated cases tended to have a higher BM blast count than DDX41-wild type cases ( $t$-test, $P=0.001$, Fig. $3 d$ ). In addition, the pre-treatment clone size of somatic DDX41 mutations was poorly correlated with BM blast count (Pearson correlation, $r=-0.07, P=0.7$, Fig. 3e). Two cases with germline DDX41 mutations showed a rapid increase of blasts 
$222(18 \%$ to $37 \%, 12 \%$ to 34\%, Fig. 3e) before therapy initiation. Somatic DDX41 mutations showed relatively small changes in clone size during treatment and their post-treatment size poorly correlated with clinical responses (Jonckheere-Terpstra test, $P=0.2$ ) or OS (log-rank test, $P=0.3$ ). This is in sharp contrast to TP53 mutations, whose clonal size showed dynamic changes and whose post-treatment clone size significantly correlated with clinical response (Jonckheere-Terpstra test, $P=2.1 \times 10^{-10}$ ) and OS (log-rank test, $P=0.001$ ) (Fig. 3f, 3g).

\section{High frequency of PPM1D-mutations in newly emerging clones}

Out of the 783 driver mutations detected in post-treatment samples, 129 from 85 cases were not detected in pre-treatment samples and thus were considered newly emerging clones. They were most commonly explained by RUNX1 mutations $(n=11)$, followed by TET2 $(n=9), C B L, D D X 41, P P M 1 D, T P 53$ $(n=8)$, and other genes. On gene basis, mutations in PPM1D (47\%), CBL (38\%), and DDX41 (29\%) were most frequently detected as newly emerging clones (Fig. 4a). Although as a whole, newly emerging clones did not necessarily predicted PD (Fig. 4b), they still correlated with treatment response depending on the type of mutated gene and their clone sizes. For example, newly emerging clones with a large (>0.30) clone size predicted poor response (5 PD and 3 SD-HI) (Fig. 4c), while those with PPM1D, TET2, and DDX41 mutations tended to have a favorable response with $88 \%(7 / 8), 75 \%$ (6/8), and $50 \%(3 / 6)$ of patients achieving CR/mCR/PR, respectively (Fig. 4d). PPMID, a TP53-induced serine/threonine phosphatase, negatively regulates TP53 pathway, whose mutations are preferentially detected in therapy-related myeloid neoplasms $(\mathrm{t}-\mathrm{MN})^{54}$ as well as in clonal hematopoiesis in healthy individuals or those who are treated by chemotherapy. In the current cohort, PPM1D mutations were found in 9 cases, including 3 cases with t-MN, in newly emerging $(n=8)$ or expanding $(n=1)$ clones during azacitidine therapy (Fig. 4e). Of particular interest is a finding that PPM1D-mutated clones coexisted with TP53-mutated clones in 6 out of the 9 cases, all of which showed a favorable molecular response to azacitidine. Single cell-sequencing analysis confirmed that TP53 and PPM1D mutations occur in distinct cells (Fig. 4f). In addition, many of these PPM1D mutations $(n=7)$ were confirmed in pre-treatment samples mostly in a small cell fraction (Extended Data Table 1). Thus, it might be speculated that in many of these patients, there was some common selective pressure that favored selection of both TP53- and PPM1D-mutated clones in a manner that initially led to the predominance of TP53-mutants and that an azacitidine-induced effacement of TP53-clones thereafter allowed for clonal expansion of pre-existing PPM1D-mutants.

\section{OS model after azacitidine therapy}

We next evaluated whether or not the multivariate model of OS constructed in Fig. 1 could be improved by including post-treatment tumor burden as expressed by the largest clone size in posttreatment sample (maxVAF ${ }^{\text {post }}$ ). In multivariate analysis, maxVAF ${ }^{\text {post }}$ was shown to be independent significant predictor of OS, together with the presence of multi-hit TP53 alteration and DDX41 mutation before therapy, best response (overall response or not), and IPSS-R score (Fig. 5a). Based on the multivariate model, we revised the scoring system by including maxVAF ${ }^{\text {post }}$ to define three risk groups (Fig. 5b). The improvement of the model was illustrated by showing a significant increment of model fitness as each of gene mutations (multi-hit TP53 alteration and DDX41 mutations)(G), clinical response $(R)$, and maxVAF ${ }^{\text {post }}(P)$ were included in the IPSS-R alone model (I) in this order (Fig. 5c). We further tested the improvement of the mode by cross-validation, in which we split the entire cohort into $75 \%$ training and $25 \%$ validation subsets and for each split, c-index was calculated for the 
validation set based on the model constructed for the training set (Fig. 5 d). The mean c-index in 10,000 cross validations increased 0.025 by adding response data to IPSS-R score (I versus IR in Fig. 5e). Addition of gene mutation and maxVAF ${ }^{\text {post }}$ data further improved prediction of the model as seen by an increased c-index by 0.034 (IR versus IGR) and 0.010 (IGR versus IGRP), respectively.

\section{Outcome of allogeneic stem cell transplantation (allo-SCT)}

Among the paired cohort, 73 patients received allo-SCT a median of 5.2 (range, 1.7-22) months after the initiation of azacitidine therapy. The median post-transplant OS was 82.6 (range, 30-58.1) months (Extended Data Fig. 8a) after a median follow-up period of 49 months. Post-treatment clone size was evaluable for 53 cases and the optimal threshold of maxVAF ${ }^{\text {post }}$ to stratify post-transplant OS was 0.13 , which significantly stratified OS after allo-SCT (log-rank test, $P=0.046$ ) (Extended Data Fig. 8b). Next, we focused on TP53-mutated cases who are expected to have a dismal prognosis even with allo-SCT ${ }^{55-}$ 57. Among 13 cases with multi-hit TP53 mutations who were transplanted, a median OS after the initiation of azacitidine therapy and allo-SCT were 24.3 and 19.2 months, respectively (Extended Data Fig. 8c, 8d). Six cases (Fig. 5e, cases 1-5, 13) were progression-free after a median follow-up of 23.7 (range, 7.3-25.0) months. Case 1 died 7 months after allo-SCT with no evidence of relapse. Notably, all 4 cases in which the mutant TP53 clone size decreased to $<0.10$ maintained $\mathrm{CR} / \mathrm{mCR}$ with a median follow-up of 23.7 (range, 7.3-24.9) months (Fig. 5f). This suggests that a significant reduction of TP53mutant clone size with azacitidine therapy could improve clinical outcomes of cases with multi-hit TP53 mutation when consolidated with allo-SCT.

\section{DISCUSSION}

NSG-based mutational profiling has successfully been applied to longitudinal disease monitoring to depict clonal dynamics in representative patients with myeloid neoplasms who were treated with a variety of drugs, including azacitidine $32,34,37,58,59$. However, the role of NGS-based profiling in the evaluation of therapeutic response has not fully been investigated in a large cohort of patients uniformly evaluated with standardized response criteria. In the current study, enrolling a large number of patients who were treated with azacitidine and uniformly assessed using the IWG criteria, we have delineated the impact of NGS-based mutation profiling of both pre- and post-treatment samples on short-term response and long-term survival after azacitidine therapy.

Compared with the conventional assessment based only on blast count and hematological recovery using the IWG criteria, NGS-based mutation profiling before and after azacitidine therapy enables a more detailed assessment of molecular response and a better prediction of OS, where the mutation status of TP53 and DDX41 and post-treatment clone size play significant roles. The mutation status of TP53 and DDX41 in pre-treatment samples improves the prediction of OS compared to using IPSS-R alone. The mutation status of both genes also improves the post-treatment OS model based only on IPSS-R and clinical response and the prediction is further improved by including post-treatment clone size (maxVAF ${ }^{\text {post }}$ ), even though the latter itself strongly correlates with clinical response, suggesting that a reduced tumor burden contributes to the efficacy of azacitidine. Of particular interest in this regard, a subset of patients who achieved CR showed a complete or almost complete clearance $(<10 \%)$ of tumor cells, or 'molecular CR', which was also seen in patients with mCR, even though hematological recovery was incomplete. Molecular CR was previously reported in decitabine-treated 
AML/MDS patients with TP53 mutations ${ }^{31}$. In our study using azacitidine, it was most frequently, though much less so than in the decitabine study, seen in patients with multi-hit TP53 mutations associated with CR/mCR responses (18/46, 39\%), but was also seen in many TP53-unmutated patients carrying RUNX1 ( $\mathrm{n}=7)$, SRSF2 ( $\mathrm{n}=7), \operatorname{TET} 2(\mathrm{n}=5)$, and DDX41 ( $\mathrm{n}=5)$ mutations in MC. Taken together, these results suggest an importance of the NGS-based assessment of post-treatment samples for evaluation of azacitidine therapy.

Despite an excellent correlation between post-treatment clone size and clinical response overall, discrepancy was seen between both in several cases. CR in most cases accompanied a substantial reduction of MCs. However, it can be achieved even with persistent large MCs having multiple mutations, typically including those affecting TET2, in a minority of cases. This confirmed a previous observation that multiple residual and/or newly emerging mutations are compatible with completely normal hematopoiesis, as reported in $\mathrm{CR}$ in $\mathrm{AML}^{60}$ or clonal hematopoiesis in healthy individuals ${ }^{47,49}$. By contrast, other cases showed PD even though a complete clearance of major clones was obtained. This was typically seen in cases with somatic DDX41 mutations. The clearance ( $<10 \%$ clone size) of somatic DDX41 mutations were found in 14 cases, of which seven achieved $\mathrm{CR} / \mathrm{mCR}$, whereas five remained SD-HI or even showed PD. However, in line with a favorable prognostic impact of DDX41 mutations in our OS models, even these cases had a relatively longer OS. Finally, while representing the major clone in most cases and associated with elevated blast counts, somatic DDX41-mutants had a significantly smaller clone sizes than expected for a major clone having other driver mutations, which together with their unique response to azacitidine, is among unique features of DDX41-mutated myeloid neoplasms. In contrast to DDX41 mutations, multi-hit TP53 lesions predict a dismal prognosis even with allo-SCT. As previously demonstrated, the excellent initial response to HMA in many cases with multi-hit TP53 lesions is not translated into an improvement of long-term outcomes, although those who achieved CR still had a significantly better OS than those who did not. Nevertheless, the excellent initial response, once obtained, might be exploited to improve long-term OS in combination with allo-SCT if performed in a timely manner. In fact, our preliminary result suggests a possibility that when performed for patients in whom the size of TP53-mutated clone was reduced to $<0.10$ before transplantation, allo-SCT could result in a long-term outcome, which needs a further evaluation.

Finally, it would be worth mentioning the emergence of newly evolving clones carrying PPM1D mutations. Although rarely found in pre-treatment samples in conventional NGS platforms $(<4 \%)$, they are frequently detected in post-treatment samples as multiple parallel mutations suggestive of strong positive selection. Of interest, PPM1D mutations, escaping mutation calls, were already present in a small fraction before therapy, indicating that such selection, if ever, would not be caused by azacitidine, but likely to be intrinsic to pre-treatment BM microenvironment. Of note in this regard, the majority (6/9) of newly emerging PPM1D mutations were found in patients with multi-hit TP53 mutations with a substantially reduced clone size in response to azacitidine, in which TP53 and PPM1D mutations are likely to be clonally independent. Because mapped on the same functional pathway, both genes are recurrently mutated in clonal hematopoiesis in association with a previous exposure to cytotoxic stress, we speculate that the similar mechanism operated in the early selection of both mutants, of PPM1Dmutants compatible with normal hematopoiesis replaced the TP53-mutant that are sensitive to azacitidine, through a bottle-neck effect. 


\section{AUTHOR CONTRIBUTIONS}

Y.N., M.Tobiasson, Y.M., E.H.-L., and S.Ogawa. conceived and designed the study. M.Tobiasson, S.S., B.E., S.Ohtake, J.T., M.C., L.Z., M.K., Y.Shibata., N.N., Mizuki W., H.Hiramoto, K.U., Mitsumasa W., K.I., H.Handa, M.Taguchi, T.K., K.O., T.I., A.K.T., H.Tsurumi, S.K., S.C., T.N., S.M., P.E., Y.M. provided patient samples and clinical data. Y.N., M.Tobiasson, J.T., Y.Shiozawa, Y.Shiraishi, H.Tanaka, K.Y., H.M., M.N., S.M., E.P., Y.M., E.H.-L., and S.Ogawa. performed bioinformatics analysis and interpreted data. Y.N., M.Tobiasson, M.C., E.H.-L., and S.Ogawa. prepared the manuscript. All authors participated in discussions and interpretation of the data and results.

\section{ACKNOWLEDGEMENTS}

This work was supported by the Japan Agency for Medical Research and Development (AMED) (JP15cm0106056h0005, JP19cm0106501h0004, JP16ck0106073h0003, JP19ck0106250h0003 to S.Ogawa.; JP19ck0106353h0003 to Y.N.; JP21ck0106470h0003 to H.M.; JP16cm0106505h to S.C.) and the Core Research for Evolutional Science and Technology (CREST) (JP19gm1110011 to S.Ogawa.); the Ministry of Education, Culture, Sports, Science and Technology of Japan; the High Performance Computing Infrastructure System Research Project (hp200138, hp210167, hp180198 and hp190158 to S.Ogawa. and S.M.); the Japan Society for the Promotion of Science (JSPS); Scientific Research on Innovative Areas (JP15H05909 to S.Ogawa. and S.M.; JP15H05912 to S.M.) and KAKENHI (JP26221308 and JP19H05656 to S.Ogawa.; JP15H05707 to S.M.; JP18H02836 to Y.N.; JP15H05668 to K.Y.; JP19H01053 to H.M.). S.Ogawa. is a recipient of the JSPS Core-to-Core Program A: Advanced Research Networks. Y.M. received grant for Clinical Cancer Research from the Ministry of Health, Labour, and Welfare of Japan (H25-GanRinsho-Ippan-006). The super-computing resource was provided by Human Genome Center, the Institute of Medical Science, the University of Tokyo. The funders of the study did not have any impact on study design, data collection, interpretation of the results or manuscript writing. We express our appreciation to Natsuki Hosho, Kazuhide Ohnishi, Rikako Onoi, Toshiko Sato, and Takeshi Shirahari for their technical assistance.

\section{COMPETING INTEREST DECLARATION}

The authors declare the following competing interests. Y.N. has received consultancy fee from Otsuka Pharmaceutical. K.U. received research funding from Otsuka, Nippon-Shinyaku, and has served on speaker bureaus for Otsuka, Nippon-Shinyaku. K.I. received honoraria from Nippon Shinyaku. T.K. received grants and lecture fees from Nippon Shinyaku. K.O received honorarium from Nippon Shinyaku. Y.M. received honorarium from Nippon Shinyaku. 
The discovery cohort included patients enrolled from 1) Japan Adult Leukemia Study Group MDS 212 (JALSG-MDS-212) study ${ }^{36}$ and 2) retrospectively collected consecutive patients treated with azacitidine at the Karolinska University Hospital. The validation cohort included retrospectively collected patients treated at Japanese institutes. The details of these cohorts are depicted in Supplementary Data. The discovery cohort and the validation cohort were analyzed separately for determining the impact of pre-treatment factors on response and survival, while a combined cohort of discovery and validation cohorts was used to explore the effect of clonal change post-treatment factors and transplantation. For details, see Supplementary Fig. 1. Patients in the discovery cohort were treated with 1 - 47 cycles of $75 \mathrm{mg} / \mathrm{m}^{2}$ azacitidine for 5 to 7 consecutive days with a median of 6 in those who have completed the treatment protocol. At the time of the last follow-up, 19 and 17 patients were still on treatment in the discovery and the validation cohorts, respectively (Extended Data Fig. 1a).

\section{Clinical data}

Data collection

For JALSG-MDS212 cohort, clinical data were collected using an electric data capture system specifically designed for this study. For Karolinska cohort, we extracted clinical data from the institutional electric health record system at Karolinska Institute. For the validation cohort, we used a questionnaire to collect clinical data from collaborative institutions.

\section{Baseline parameter}

401 Baseline parameters included patient age at azacitidine administration, sex, history of chemotherapy 402 or radiotherapy for other malignancies, disease subtype according to WHO 2016 classification ${ }^{61}$, risk 403 category by IPSS-R (not applied to chronic myelomonocytic leukemia (CMML) cases with WBC 12.0 $\left.404 \times 10^{9} / L\right)$, karyotype (KT), absolute neutrophil counts (ANC), hemoglobin, platelet and bone marrow 405 (BM) blast ratio. KT risk was evaluated according to IPSS-R-based KT risk classification ${ }^{62}$ and classified 406 into higher (poor and very poor) and lower (the others) groups. Continuous variables from laboratory 407 data were divided into lower and higher groups by clinically significant thresholds in reference to the 408 criteria applied in IPSS- $R^{62}$. Specifically, ANC $<8.0 \times 10^{9} / \mathrm{L}$, hemoglobin level $<80 \mathrm{~g} / \mathrm{L}$, platelet counts $<$ $40950 \times 10^{9} / \mathrm{L}$, were assumed lower and BM blast ratio $>10 \%$ as higher. Age was classified into higher and 410 lower groups by the median value of 72-year-old in the entire cohort. We did not include marrow 411 cellularity because bone marrow biopsy was not conducted in many Japanese samples. We also 412 excluded transfusion dependencies from the analyses because transfusion policy is different between 413 Japan and Sweden. IPSS-R was calculated using the laboratory data before azacitidine administration.

\section{Response assessment.}

415 Treatment response was assessed according to the International Working Group (IWG) response 416 criteria $2006^{63}$. The best response that was achieved from azacitidine treatment and the response at 417 the time of post-treatment sampling were recorded. The former was assessed except for the analysis 418 that explored the correlation between post-treatment clone size and clinical response. Patients 419 without response information were not included in the section that explore the factors predicting 
clinical response. Hematological improvement (HI) included achieving improvement in either of erythroid (HI-E), platelet (HI-P) or neutrophil (HI-N) lineage.

422

423

424

425

426

427

428

429

430

431

432

433

434

435

436

437

438

439

440

441

442

443

444

445

446

447

448

449

450

451

452

453

454

455

456

457

458

\section{Targeted sequencing}

\section{Sampling of tumor cells}

For JALSG-MDS212 cohort, bone marrow was collected just before start of azacitidine treatment and after 4 courses according to the study protocol unless patients were dropped out before course 4 . Bone marrow samples were collected at LSI Medience Corporation, where DNA was extracted and stored. For Karolinska cohort and the validation cohort, bone marrow samples collected and analyzed before course 1 and around course 4 (Extended Data Fig. 1c). In this paper, we refer to the samples collected before azacitidine administration and around course 4 as 'pre-treatment' and 'posttreatment' samples, respectively.

\section{Panel information for targeted-capture sequencing}

We applied targeted-capture sequencing using either of 5 panels designed for genetic study of myeloid malignancies at Kyoto University $(n=4)$ or Memorial Sloan Kettering Cancer Center (MSKCC) $(n=1)$. 66 genes were commonly included in all the panels and were investigated in this study (Supplementary Table 1). All the panels also included 1,158 to 1,428 SNPs probes so that we can detect genome-wide copy number changes and allelic imbalances.

\section{Sequencing method}

97 of 163 pre-treatment samples from Karolinska Institute cohort were sequenced at MSKCC lab. For library construction, 11 to 800 ng of genomic DNA was used using the KAPA Hyper Prep Kit (Kapa Biosystems KK8504) with 7 to 12 cycles of PCR. After sample barcoding, 10 to 1,610 ng of each library were pooled and captured by hybridization. Captured pools were sequenced with paired-end Illumina HiSeq at 100 bp or 125 bp read length. A set of normal control was built by sequencing 48 blood samples from cases who did not have hematological disease. Fastq files were shared with Kyoto University Lab. All the other samples were sequenced at Kyoto University. 50 ng or 200 ng of genomic DNA were enriched for target regions by liquid-phase hybridization using the SureSelect custom kit (Agilent Technologies ${ }^{\circledR}$ ), according to the manufacturer's protocol optimized for automated sample processing, as previously described ${ }^{64}$. Purified library was subjected to high-throughput sequencing analysis with Illumina HiSeq 2500 or NovaSeq 6000 using 125 bp or 150 bp pair-end mode. We also sequenced 106 blood samples from the subjects who do not have hematological diseases and used as normal controls for exclusion of sequencing errors.

\section{Mutation call}

Sequencing reads were aligned to the human genome reference (hg19) using Burrows-Wheeler Aligner, version 0.7.8 with default parameter settings. All the bam files generated at MSKCC and Kyoto University were collected and analyzed together at Kyoto University. Quality of the sequencing data are summarized in Supplementary Fig. 2. Mutation calling was performed through our established pipeline (genomon pipeline 2.6.0, https://github.com/Genomon-Project), as previously reported ${ }^{64-67}$ using the following parameters.

Adopt variants fulfilling the following criteria: 
(ii) Base Quality score $\geq 15$

(iii) Number of total reads $\geq 100$

(iv) Number of variant reads $\geq 4$

(v) Variant allele frequency $\geq 0.02$

Following candidates were excluded:

(i) Synonymous and ambiguous (unknown) variants

(ii) Variants which were read only from one direction

(iii) Single nucleotide substitutions in which other mutations were called at the same position and their variant allele frequency was $\geq 0.1$.

Mapping errors were removed by visual inspection on the Integrative Genomics Viewer (IGV) browser (http://software.broadinstitute.org/software/igv/). Structural variants were also called using genomon pipeline 2.6.0.

\section{Curation of oncogenic variants}

The significant variants that fulfill the quality filter noted above were further assessed for oncogenicity based on in-house curation program. The curation policy was determined individually for each gene based on previous reports and databases after exclusion of variants registered in public SNPS databases (the 1000 genomes project as of 2014 Aug, ESP6500, Human Genome Variation Database) and call errors using EB call ${ }^{68}$ and in-house blacklist of error calls. All the pathogenic calls are listed in Supplementary Table 2 and 3.

\section{Copy number and allelic imbalance}

We included 1,158 to 1,428 SNPs probes to allow for detection of copy number changes and allelic imbalances. This technique, called CNACS is implemented in the program available at https://github.com/papaemmelab/toil cnacs. Manual inspection of the result was conducted to discriminate call errors.

\section{CNAs and definition of arm level CNAs}

Total copy number (TCN) of 2.22 or larger was assumed $\mathrm{CN}$-gains, and TCN $<1.88$ was assumed $\mathrm{CN}$-loss. Copy number neutral LOH was called when B-allele frequency was $<0.90$ with TCN between 1.88 and 2.22. Arm level CNA were called when the total length of affected region within the arm is $>1 \mathrm{M} \mathrm{bp}$ for $17 p$ and $>3 \mathrm{M}$ bp for the other arms. For gains in chromosome 8, CNA events in long and short arms are counted together. Partial tandem duplication in KMT2A (KMT2A-PTD) was detected using CNA data. Specifically, KMT2A-PTD was called when copy number gain (TCN > 2.5) was detected within the region between intron 1 and intron 19 of KMT2A (NM_005933) gene. All the CNAs are listed in Supplementary Tables 4 and 5.

\section{Calculation of clone size for each mutation}

The clone size of the relevant mutation was calculated from VAF values accounting for chromosomal ploidy considering sex, copy number changes, and allelic imbalances that span the mutated region. The details of adjustment calculation are described before $\mathrm{e}^{55}$. 


\section{Germline versus somatic origins of DDX41 mutations}

DDX41 represents one of the largest inheritable risk for myeloid malignancies and about 50 to $79 \%$ of the MDS or AML patients with DDX41 germline variants acquire somatic mutations in DDX41 gene $e^{42,43}$. The DDX41 variants were assumed somatic when the variant allele frequency (VAF) value was $<0.34$ for indels and $<0.39$ for single nucleotide variants (SNVs). For the cases with CNAs in the DDX41 region, CNA-adjusted VAF was calculated. All the others were judged likely germline. In the analyses of evaluating clone size changes after azacitidine administration, only somatic mutations were considered. The DDX41 variants were listed with VAF values in Supplementary Table 6.

\section{Curation of mutations in TP53}

We consulted IARC TP53 database (http://p53.iarc.fr/) for pathogenicity ${ }^{69}$. Specifically, we checked the transcriptional activity (TA) for each SNVs in the 'Gene variation' database, which summarizes the change of TA on 8 different promoters by introducing artificially constructed vectors harboring all possible amino acid substitutions $(n=2,314)$ into yeast $^{70}$. Interestingly, we found that none of the nondamaging mutations in this database were associated with either loss of heterozygosity (LOH) in 17p or complex karyotype unless they possessed other pathogenic TP53 mutations. Finally, we judged the alterations is the following list as non-pathogenic: E11Q, V31I, A76G, A129P, A189V, N235S, N310K, G360V. We assumed all the mutations resulting in gene truncation and those in the splicing regions as pathogenic. In addition, we discriminated multi-hit cases from mono-allelic case based on the presumed number of alleles affected. Briefly, multi-hit mutation is judged when a patient has either of 1) two of more TP53 mutations, 2) one TP53 mutation accompanied by either of copy number neutral LOH or deletion of 17p. The significance of discriminating these two allelic statuses of TP53 mutation is described in our recent paper ${ }^{37}$.

\section{Definition of major clones (MC)}

In the analysis of the clonal changes, FLT3-ITD and KMT2A-PTD were removed from the mutation list because the mutated clone size cannot be adequately assessed with targeted-sequencing data for these alterations. The germline DDX41 mutations were also removed. All the mutations were classified into $\mathrm{MC}$ or non-MC. First, mutated clone size was assessed for all the mutations detected in pretreatment samples, and the largest clone group in pre-treatment samples ( $\mathrm{MC}^{\mathrm{pre}}$ ) was defined to include the largest and near largest clones (with the difference in clone size from the largest one being $<0.10$. This threshold corresponds to difference of 0.05 in VAF sizes for autosomes without CNAs). Next, mutated clone size in post-treatment samples was assessed for all $M^{\text {pre }}$ and the subset of $M^{\text {pre }}$ showing the largest and near largest size after treatment were defined as MC. Again, the difference in clone size of near largest clones from the largest one was set $<0.10$. Specifically, we assigned 448 $M^{\text {pre }}$ and $387 \mathrm{MC}$ among 851 mutated clones detected in pre-treatment samples. A set of MCs in a case was defined as a 'MC group' and its size was defined to be the average value of the clones included in the MC group (Fig. 2a). Note that post-treatment size of $M C$ group can be 0 when all $M C^{\text {pre }}$ in a case disappeared after treatment, and actually this was achieved in 44 cases.

\section{Single-cell sequencing library preparation and genotyping}

Patient samples were thawed and washed with PBS supplemented with EDTA and 5\% FCS (FACS buffer). Cells were stained with APC-Cy7-conjugated CD45 for $15 \mathrm{~min}$ at $4{ }^{\circ} \mathrm{C}$. Cells were then washed and resuspended in FACS buffer with DAPI and sorted to isolate viable blood cells (DAPI-, CD45+) using 
540 FACSAria III Cell Sorter (Beckton \& Dickinson). Single cells were encapsulated using a Tapestri

541 microfluidics cartridge, lysed, and barcoded. Barcoded samples were then subjected to targeted PCR

542 amplification of a custom 312 amplicons covering 45 genes implicated in myeloid malignancies

543 included in Tapestri ${ }^{\circledR}$ Single-Cell DNA Myeloid Panel. PCR products were merged, purified with Ampure

544 XP beads (Beckman Coulter), and used as a template for PCR to incorporate Illumina i5/i7 indices. PCR

545 products were quantified using TapeStation 4400 (Agilent Technology) and subjected to sequencing

546 on an Illumina NovaSeq 6000 (Illumina). FASTQ files were analyzed through the Tapestri pipeline to

547 generate a loom file. Filtering low-quality genotypes or cells and defining clones were performed with

548 Tapestri Insights following manufacturer's instruction.

\section{Whole-genome sequencing}

55050 ng DNA were subjected to library preparation using KAPA Hyper Prep Kit followed by sequencing 551 using DNBSEQ-G400 (MGI Tech) with target depth of 100x in 150-bp paired-end mode. The target 552 depth was 100x, and the actual depth was $133 \times(60-293 \times)$. The mean depth in germline controls from 553122 individuals was $140 \times(55-204 \times)$. Mutation calling was performed using the Genomon2 pipeline 554 (v.2.6), as previously described ${ }^{71}$. Somatic mutations were detected by eliminating polymorphisms and 555 sequencing errors. To achieve this, we first applied default Genomon 2.6.0 settings and further 556 removed low-quality, unreliable reads and variants according to the following criteria: (i) a sufficient 557 number of reads (total reads $>30$ and variant reads $>3$ ), (ii) variant alleles are observed at a 558 significantly higher frequency than expected by error (number of supporting reads in controls $<5$, 559 Fisher $P$-value $<10^{-1}$ ), (iii) significant variants on the basis of an empirical distribution of VAFs as 560 determined using WGS data of non-paired peripheral blood samples (EBCall algorithm, $n=16$ ) ${ }^{68}$, (iv) 561 not included in common SNPs (frequency in 1000 genome project 2014 Oct all database $<0.001$, 562 https://www.internationalgenome.org/). Only single nucleotide variants are assumed.

\section{Statistical analysis}

564 All statistical calculations were performed using $\mathrm{R}$ version 4.0.3. Differences in distribution of 565 categorical and continuous variables were assessed with Fisher's exact test and Student's t-test, 566 respectively. All the explanatory variables were dichotomized if not specified using the thresholds 567 presented above. Time was assessed from the start of azacitidine treatment to the events of interest 568 unless otherwise specified. Time-to-event data were analyzed using the Kaplan-Meier method and 569 assessed for difference among groups by log-rank test using survival_3.2-7 package. For the analyses 570 to explore the factors associated with clinical response, Fisher's exact test and logistic regression 571 model was applied for univariate and multivariate analysis, respectively. For the analyses to explore 572 the factors associated with survival, Cox proportional hazard model was applied for both univariate 573 and multivariate model. Significant and sub-significant $(P<0.10)$ factors in univariate analyses with 574 incidence of $>10 \%$ in the target cohort were subjected to a multivariate analysis followed by 575 parameter selection with Akaike's information criteria (AIC) using step function in R base functions. 576 Multiple collinearity among explicative variables was assessed before parameter selection by 577 calculating generalized variance-inflation factor (gVIF) using vif function of car_3.0-10 package for the 578 multivariate model. If the largest gVIF is $>3$, the explicative variable showing the second largest gVIF 579 value was removed and reconstructed the multivariate model with the residual variables. Stratified OS 580 model was constructed using the covariates that were significant in multivariate analysis. The weight 581 of each covariate was determined based on the coefficients of Cox proportional hazard model 
582

583

584

585

586

587

588

589

590

591

592

593

594

595

596

597

598

599

600

601

602

603

604
( $\log ($ hazard ratio)) with a scaling factor and large rounding. The cut off values for risk stratification were determined on the basis of grid search for the two thresholds that most significantly stratified the discovery cohort into three groups in terms of OS. Differences in likelihood between two regression models were compared by anova. Concordance index (c-index) of survival data was calculated using estC function of compareC_1.3.1 package. The optimal cut point of max (VAF ${ }^{\text {post }}$ ) was determined by surv_cutpoint function of survminer_0.4.8 package. In addition, $\mathrm{R}$ packages ggplot2_3.3.2, forestmodel_0.6.2, ggpubr_0.4.0, ggsci_2.9, patchwork_1.0.1, RColorBrewer_1.1-2 packages were used for graphical presentation.

\section{Ethical issue}

This study was approved by the institutional ethical committee at Kyoto University (G0608), Karolinska Institute (Dnr 2017/1090-31/4) and all the participating institutes and hospitals. All patients provided fully informed consent.

\section{Data accessibility}

Tables containing clinical data, results of univariate analysis for response or OS, clone size in pre-and post-treatment samples are available on Github at https://github.com/ynanya/MDS-postAZA-clone.

\section{Code reproducibility}

All computational code is available at https://github.com/ynanya/MDS-postAZA-clone. 


\section{REFERENCES}

1. Silverman, L.R., et al. Randomized controlled trial of azacitidine in patients with the myelodysplastic syndrome: a study of the cancer and leukemia group B. J Clin Oncol 20, 2429-2440 (2002).

2. Papageorgiou, S.G., et al. Treatment with 5-Azacytidine improves clinical outcome in highrisk MDS patients in the 'real life' setting: A single center observational study. Hematology 21, 34-41 (2016).

3. Fenaux, P., et al. Efficacy of azacitidine compared with that of conventional care regimens in the treatment of higher-risk myelodysplastic syndromes: a randomised, open-label, phase III study. Lancet Oncol 10, 223-232 (2009).

4. Itzykson, R., et al. Impact of TET2 mutations on response rate to azacitidine in myelodysplastic syndromes and low blast count acute myeloid leukemias. Leukemia 25, 1147-1152 (2011).

5. Itzykson, R., et al. Prognostic factors for response and overall survival in 282 patients with higher-risk myelodysplastic syndromes treated with azacitidine. Blood 117, 403-411 (2011).

6. Simo-Riudalbas, L., Melo, S.A. \& Esteller, M. DNMT3B gene amplification predicts resistance to DNA demethylating drugs. Genes Chromosomes Cancer 50, 527-534 (2011).

7. Voso, M.T., et al. Role of BCL2L10 methylation and TET2 mutations in higher risk myelodysplastic syndromes treated with 5-azacytidine. Leukemia 25, 1910-1913 (2011).

8. Itzykson, R. \& Fenaux, P. Predicting the outcome of patients with higher-risk myelodysplastic syndrome treated with hypomethylating agents. Leuk Lymphoma 53, 760-762 (2012).

9. Bally, C., et al. Prognostic value of TP53 gene mutations in myelodysplastic syndromes and acute myeloid leukemia treated with azacitidine. Leuk Res 38, 751-755 (2014).

10. Bejar, R., et al. TET2 mutations predict response to hypomethylating agents in myelodysplastic syndrome patients. Blood 124, 2705-2712 (2014).

11. DiNardo, C.D., et al. Lack of association of IDH1, IDH2 and DNMT3A mutations with outcome in older patients with acute myeloid leukemia treated with hypomethylating agents. Leuk Lymphoma 55, 1925-1929 (2014).

12. Traina, F., et al. Impact of molecular mutations on treatment response to DNMT inhibitors in myelodysplasia and related neoplasms. Leukemia 28, 78-87 (2014).

13. Desoutter, J., et al. Molecular prognostic factors in acute myeloid leukemia receiving first-line therapy with azacitidine. Leukemia 30, 1416-1418 (2016).

14. Jung, S.H., et al. Somatic mutations predict outcomes of hypomethylating therapy in patients with myelodysplastic syndrome. Oncotarget 7, 55264-55275 (2016).

15. Merlevede, J., et al. Mutation allele burden remains unchanged in chronic myelomonocytic leukaemia responding to hypomethylating agents. Nat Commun 7, 10767 (2016).

16. Takahashi, K., et al. Clinical implications of TP53 mutations in myelodysplastic syndromes treated with hypomethylating agents. Oncotarget 7, 14172-14187 (2016).

17. Tobiasson, M., et al. Mutations in histone modulators are associated with prolonged survival during azacitidine therapy. Oncotarget 7, 22103-22115 (2016).

18. Cedena, M.T., et al. Mutations in the DNA methylation pathway and number of driver mutations predict response to azacitidine in myelodysplastic syndromes. Oncotarget 8 , 106948-106961 (2017).

19. Hiller, J.K., et al. Evaluating the impact of genetic and epigenetic aberrations on survival and response in acute myeloid leukemia patients receiving epigenetic therapy. Ann Hematol 96, 559-565 (2017).

20. Unnikrishnan, A., et al. Integrative Genomics Identifies the Molecular Basis of Resistance to Azacitidine Therapy in Myelodysplastic Syndromes. Cell Rep 20, 572-585 (2017). 
21. Dohner, H., et al. Cytogenetics and gene mutations influence survival in older patients with acute myeloid leukemia treated with azacitidine or conventional care. Leukemia 32, 25462557 (2018).

22. Kuendgen, A., et al. Efficacy of azacitidine is independent of molecular and clinical characteristics - an analysis of 128 patients with myelodysplastic syndromes or acute myeloid leukemia and a review of the literature. Oncotarget 9, 27882-27894 (2018).

23. Wang, H., et al. Predictors of clinical responses to hypomethylating agents in acute myeloid leukemia or myelodysplastic syndromes. Ann Hematol 97, 2025-2038 (2018).

24. Falconi, G., et al. Somatic mutations as markers of outcome after azacitidine and allogeneic stem cell transplantation in higher-risk myelodysplastic syndromes. Leukemia 33, 785-790 (2019).

25. Qin, Y., Kuang, P., Zeng, Q., Wu, Y. \& Liu, T. Hypomethylating agents for patients with myelodysplastic syndromes prior to hematopoietic stem cell transplantation: a systematic review and meta-analysis. Ann Hematol 98, 2523-2531 (2019).

26. Wu, P., et al. Co-occurrence of RUNX1 and ASXL1 mutations underlie poor response and outcome for MDS patients treated with HMAs. Am J Transl Res 11, 3651-3658 (2019).

27. Martin, I., et al. Impact of clinical features, cytogenetics, genetic mutations, and methylation dynamics of CDKN2B and DLC-1 promoters on treatment response to azacitidine. Ann Hematol 99, 527-537 (2020).

28. Walter, M.J., et al. Clonal architecture of secondary acute myeloid leukemia. N Engl J Med 366, 1090-1098 (2012).

29. Font, P., et al. Inter-observer variance with the diagnosis of myelodysplastic syndromes (MDS) following the 2008 WHO classification. Ann Hematol 92, 19-24 (2013).

30. Platzbecker, U., et al. Proposals for revised IWG 2018 hematological response criteria in patients with MDS included in clinical trials. Blood 133, 1020-1030 (2019).

31. Welch, J.S., et al. TP53 and Decitabine in Acute Myeloid Leukemia and Myelodysplastic Syndromes. N Engl J Med 375, 2023-2036 (2016).

32. Uy, G.L., et al. Dynamic changes in the clonal structure of MDS and AML in response to epigenetic therapy. Leukemia 31, 872-881 (2017).

33. Woo, J., et al. Mutational analysis in serial marrow samples during azacitidine treatment in patients with post-transplant relapse of acute myeloid leukemia or myelodysplastic syndromes. Haematologica 102, e216-e218 (2017).

34. Calleja, A., et al. Clonal selection in therapy-related myelodysplastic syndromes and acute myeloid leukemia under azacitidine treatment. Eur J Haematol 104, 488-498 (2020).

35. Duchmann, M., et al. Prognostic Role of Gene Mutations in Chronic Myelomonocytic Leukemia Patients Treated With Hypomethylating Agents. EBioMedicine 31, 174-181 (2018).

36. Kiguchi, T., et al. Prospective Comparison of Azacitidine Treatment between 7-Days and 5Days Schedules for Patients with Higher-Risk Myelodysplastic Syndromes; Results of Japan Adult Leukemia Study Group MDS212 Trial. in 61st The American Society of HematologyAnnual Meeting, Vol. 134 (Orland, 2019).

37. Bernard, E., et al. Implications of TP53 allelic state for genome stability, clinical presentation and outcomes in myelodysplastic syndromes. Nat Med 26, 1549-1556 (2020).

38. Kurumaddali, A., Salem, A.H. \& Agarwal, S.K. A Meta-Analysis of Higher-risk Myelodysplastic Syndrome Trials to Evaluate the Relationship between Short-term Endpoints and Overall Survival. J Cancer 10, 5427-5433 (2019).

39. Montalban-Bravo, G., et al. Genomic context and TP53 allele frequency define clinical outcomes in TP53-mutated myelodysplastic syndromes. Blood Adv 4, 482-495 (2020).

40. Sallman, D.A., et al. Impact of TP53 mutation variant allele frequency on phenotype and outcomes in myelodysplastic syndromes. Leukemia 30, 666-673 (2016).

41. Welch, J.S., Petti, A.A. \& Ley, T.J. Decitabine in TP53-Mutated AML. N Engl J Med 376, 797798 (2017). 
42. Polprasert, C., et al. Inherited and Somatic Defects in DDX41 in Myeloid Neoplasms. Cancer Cell 27, 658-670 (2015).

43. Sebert, M., et al. Germline DDX41 mutations define a significant entity within adult MDS/AML patients. Blood 134, 1441-1444 (2019).

44. Quesada, A.E., et al. DDX41 mutations in myeloid neoplasms are associated with male gender, TP53 mutations and high-risk disease. Am J Hematol 94, 757-766 (2019).

45. Breccia, M., Salaroli, A., Loglisci, G. \& Alimena, G. Revised IPSS (IPSS-R) stratification and outcome of MDS patients treated with azacitidine. Ann Hematol 92, 411-412 (2013).

46. Lamarque, M., et al. The revised IPSS is a powerful tool to evaluate the outcome of MDS patients treated with azacitidine: the GFM experience. Blood 120, 5084-5085 (2012).

47. Jaiswal, S., et al. Age-related clonal hematopoiesis associated with adverse outcomes. $N$ Engl J Med 371, 2488-2498 (2014).

48. Xie, M., et al. Age-related mutations associated with clonal hematopoietic expansion and malignancies. Nat Med 20, 1472-1478 (2014).

49. Genovese, G., et al. Clonal hematopoiesis and blood-cancer risk inferred from blood DNA sequence. N Engl J Med 371, 2477-2487 (2014).

50. Forghieri, F., et al. NPM1 mutations may reveal acute myeloid leukemia in cases otherwise morphologically diagnosed as myelodysplastic syndromes or myelodysplastic/myeloproliferative neoplasms. Leuk Lymphoma 56, 3222-3226 (2015).

51. Makishima, H., et al. Dynamics of clonal evolution in myelodysplastic syndromes. Nat Genet 49, 204-212 (2017).

52. Nagata, Y., et al. Invariant patterns of clonal succession determine specific clinical features of myelodysplastic syndromes. Nat Commun 10, 5386 (2019).

53. Wu, L., et al. NPM1 mutation with DNMT3A wild type defines a subgroup of MDS with particularly favourable outcomes after decitabine therapy. Br J Haematol 189, 982-984 (2020).

54. Hsu, J.I., et al. PPM1D Mutations Drive Clonal Hematopoiesis in Response to Cytotoxic Chemotherapy. Cell Stem Cell 23, 700-713 e706 (2018).

55. Yoshizato, T., et al. Genetic abnormalities in myelodysplasia and secondary acute myeloid leukemia: impact on outcome of stem cell transplantation. Blood 129, 2347-2358 (2017).

56. Bejar, R., et al. Somatic mutations predict poor outcome in patients with myelodysplastic syndrome after hematopoietic stem-cell transplantation. J Clin Oncol 32, 2691-2698 (2014).

57. Lindsley, R.C., et al. Prognostic Mutations in Myelodysplastic Syndrome after Stem-Cell Transplantation. N Engl J Med 376, 536-547 (2017).

58. da Silva-Coelho, P., et al. Clonal evolution in myelodysplastic syndromes. Nat Commun 8 , 15099 (2017).

59. Mossner, M., et al. Mutational hierarchies in myelodysplastic syndromes dynamically adapt and evolve upon therapy response and failure. Blood 128, 1246-1259 (2016).

60. Rothenberg-Thurley, M., et al. Persistence of pre-leukemic clones during first remission and risk of relapse in acute myeloid leukemia. Leukemia (2017).

61. WHO Classificaton of Tumors of Haematopoietic and Lymphoid Tissues, Revised 4th Edition., (International Agency for Research on Cancer, Lyon, 2017).

62. Greenberg, P.L., et al. Revised international prognostic scoring system for myelodysplastic syndromes. Blood 120, 2454-2465 (2012).

63. Cheson, B.D., et al. Clinical application and proposal for modification of the International Working Group (IWG) response criteria in myelodysplasia. Blood 108, 419-425 (2006).

64. Yoshida, K., et al. Frequent pathway mutations of splicing machinery in myelodysplasia. Nature 478, 64-69 (2011).

65. Haferlach, T., et al. Landscape of genetic lesions in 944 patients with myelodysplastic syndromes. Leukemia 28, 241-247 (2014). 
66. Yoshizato, T., et al. Somatic Mutations and Clonal Hematopoiesis in Aplastic Anemia. The

67. Suzuki, H., et al. Mutational landscape and clonal architecture in grade II and III gliomas. Nature genetics 47, 458-468 (2015).

68. Shiraishi, Y., et al. An empirical Bayesian framework for somatic mutation detection from cancer genome sequencing data. Nucleic Acids Res 41, e89 (2013).

69. Bouaoun, L., et al. TP53 Variations in Human Cancers: New Lessons from the IARC TP53 Database and Genomics Data. Hum Mutat 37, 865-876 (2016).

70. Kato, S., et al. Understanding the function-structure and function-mutation relationships of p53 tumor suppressor protein by high-resolution missense mutation analysis. Proc Natl Acad Sci U S A 100, 8424-8429 (2003).

71. Yokoyama, A., et al. Age-related remodelling of oesophageal epithelia by mutated cancer drivers. Nature 565, 312-317 (2019). 


\section{TABLES, FIGURES AND EXTENDED DATA LEGENDS}

Table 1 | Characteristics of the study cohort.

Figure 1 | Genetic and clinical factors associated with response and overall survival.

Figure 2 | Clone changes during azacitidine treatment and genetic mechanisms of CR.

Figure 3 | Somatic DDX41-mutated clone size does not correlate with blast ratio or response to treatment.

Figure 4 | Property and role of newly emerging clones after azacitidine treatment.

Figure 5 | The role of post-treatment clone size on improvement of OS model and predicting posttransplant outcomes.

Extended Data Figure 1 | Azacitidine treatment profile, overall survival and time to response.

Extended Data Figure 2 | Factors associated with achieving complete remission (CR).

Extended Data Figure 3 | Factors associated with achieving overall response (OR) and having progressive disease (PD).

Extended Data Figure 4 | Univariate analysis for overall survival (OS).

Extended Data Figure 5 | Factors associated with overall survival (OS).

Extended Data Figure 6 | Major clone size in pre-treatment samples and clonal structure in stable disease (SD) cases with small post-treatment major clone (MC) size.

Extended Data Figure 7 | Whole genome sequencing to search for potential drivers.

Extended Data Figure 8 | Outcomes of transplanted cases.

Extended Data Table 1 | Strenuous search for PPM1D-mutated clones by integrative genomics viewer (IGV) in pre-treatment samples that expanded their sizes in post-treatment samples. 
Table 1. Characteristics of the study cohort

\begin{tabular}{|c|c|c|c|c|c|c|c|c|}
\hline & \multicolumn{3}{|c|}{ Discovery cohort } & \multicolumn{3}{|c|}{ Validaiton cohort } & \multicolumn{2}{|l|}{ P-values } \\
\hline No. of patients & 341 & & & 110 & & & & \\
\hline Age at enrollement (median [range]) - yrs & 72 & ( 19-91 & ) & 69 & $(16-84$ & ) & 0.0011 & * \\
\hline Gender - no. $(\%)$ & & & & & & & 0.10 & $\dagger$ \\
\hline Male & 224 & 65.7 & ) & 82 & 74.5 & ) & & \\
\hline Female & 117 & 34.3 & ) & 28 & $(25.5$ & ) & & \\
\hline WHO-classification at enrollment -no. (\%) & & & & & & & 0.024 & $\dagger \ddagger$ \\
\hline MDS & 295 & 86.5 & ) & 91 & 82.7 & ) & & \\
\hline isolated del $(5 q)$ & 3 & 0.9 & ) & 2 & 1.8 & ) & & \\
\hline MDS-SLD & 1 & 0.3 & ) & 1 & 0.9 & ) & & \\
\hline MDS-MLD & 19 & 5.6 & ) & 18 & 16.4 & ) & & \\
\hline MDS-RS & 1 & 0.3 & ) & 3 & 2.7 & ) & & \\
\hline MDS-U & 4 & 1.2 & ) & 0 & 0.0 & ) & & \\
\hline MDS-EB1 & 123 & 36.1 & ) & 26 & 23.6 & ) & & \\
\hline MDS-EB2 & 144 & 42.2 & ) & 41 & 37.3 & ) & & \\
\hline $\mathrm{AML}$ & 21 & 6.2 & ) & 15 & 13.6 & ) & & \\
\hline MDS/MPN & 25 & 7.3 & ) & 4 & 3.6 & ) & & \\
\hline Atypical CML, BCR-ABL1 negative & 0 & 0.0 & ) & 1 & 0.9 & ) & & \\
\hline CMML & 21 & 6.2 & ) & 2 & 1.8 & ) & & \\
\hline MDS/MPN-U & 4 & 1.2 & ) & 1 & 0.9 & ) & & \\
\hline IPSS-R - no. $(\%)^{\S}$ & & & & & & & 0.14 & $\dagger$ \\
\hline Very Low & 1 & 0.3 & ) & 3 & 2.8 & ) & & \\
\hline Low & 11 & 3.4 & ) & 6 & 5.7 & ) & & \\
\hline Intermediate & 73 & 22.8 & ) & 19 & 17.9 & ) & & \\
\hline High & 96 & 30.0 & ) & 30 & 28.3 & ) & & \\
\hline Very High & 139 & 43.4 & ) & 48 & 45.3 & ) & & \\
\hline Karyotype risks (IPSS-R-based) - no. (\%) & & & & & & & 0.021 & $\dagger$ \\
\hline Very Good & 3 & 0.9 & ) & 5 & 4.4 & ) & & \\
\hline Good & 148 & 43.5 & ) & 36 & 33.6 & ) & & \\
\hline Intermediate & 63 & 18.5 & ) & 16 & $(15.0$ & ) & & \\
\hline Poor & 38 & 11.2 & ) & 16 & 14.2 & ) & & \\
\hline Very Poor & 88 & $(\quad 25.9$ & ) & 37 & $(\quad 32.7$ & ) & & \\
\hline \multicolumn{9}{|l|}{ Peripheral Blood } \\
\hline WBC (median (range)) $\times 10^{9} / \mathrm{L}$ & 2.8 & $(0.3-107.9$ & & 2.4 & $(0.4-57.8$ & ) & 0.044 & * \\
\hline ANC (median (range)) $\times 10^{9} / \mathrm{L}$ & 1.0 & $(0-38.2$ & ) & 1.0 & $(0.05-31.8$ & ) & 0.39 & * \\
\hline $\mathrm{HB}$ (median (range)) $\mathrm{g} / \mathrm{dL}$ & 9.2 & $(4.1-15$ & ) & 8.2 & $(4.2-14.9$ & ) & 0.0014 & * \\
\hline PLT (median (range)) $\times 10^{9} / \mathrm{L}$ & 69.0 & $(5-1237$ & ) & 73.5 & $(4-709$ & ) & 0.93 & * \\
\hline \multicolumn{9}{|l|}{ Bone Marrow } \\
\hline Blast (median [range]) - \% & 10 & $(0-37.0$ & ) & 9.0 & $(0-30.0$ & ) & 0.33 & * \\
\hline Post aza samples - available no. (\%) & 237 & 69.5 & ) & 53 & 48.18 & ) & 0.00017 & $\dagger$ \\
\hline RBC transfusion dependent - no. (\%) & 166 & 48.7 & ) & 47 & 44.3 & ) & 0.44 & $\dagger$ \\
\hline PLT transfusion dependent - no. (\%) & 29 & 8.5 & ) & 19 & 17.6 & ) & 0.012 & $\dagger$ \\
\hline Response (best response) & & & & & & & 0.0005 & $\dagger$ \\
\hline $\mathrm{CR}(\%)$ & 59 & 19.0 & ) & 13 & 14.9 & ) & & \\
\hline mCR (\%) & 69 & 22.2 & ) & 6 & 6.9 & ) & & \\
\hline PR (\%) & 2 & 0.6 & ) & 19 & ( $\quad 21.8$ & ) & & \\
\hline SD (\%) & 110 & 35.4 & ) & 33 & 37.9 & ) & & \\
\hline $\mathrm{PD}(\%)$ & 71 & 22.8 & ) & 16 & ( 18.4 & ) & & \\
\hline $\begin{array}{l}\text { *Student's t-test was applied to compare cor } \\
\text { Distribution among major categories are asse } \\
\text { Abbreviations: MDS-SLD, MDS with single lir } \\
\text { anemia with ring sideroblasts; MDS-U, MDS } \\
\text { syndromes; AML, acute myeloid leukemia; M } \\
\text { leukemia; CMML, chronic myelomonocytic le } \\
\text { IPSS-R, International Prognostic Scoring Sys } \\
\text { hemoglobine; PLT, platelet; CR, complete rer } \\
\text { progressive disease. }\end{array}$ & $\begin{array}{l}\text { Ialues. } \dagger \\
\text { PSS-R v } \\
\text { splasia; I } \\
\text { able; ML } \\
\text { Myelod } \\
\text { MDS/MP } \\
\text { sed; WB } \\
\text { nCR, me }\end{array}$ & $\begin{array}{l}\text { Fisher's exac } \\
\text { las not calcul } \\
\text { ADS-MLD, M } \\
\text { S-EB1/2, ML } \\
\text { /splastic/mye } \\
\text { V-U, Myelody } \\
\text { C, white bloo } \\
\text { rrow CR; PR }\end{array}$ & $\begin{array}{l}\text { late } \\
\text { DS } \\
\text { DS v } \\
\text { lop } \\
\text { spl } \\
\mathrm{d} \mathrm{ce} \\
\text { pa }\end{array}$ & $\begin{array}{l}\text { pplied } \\
\text { IML cas } \\
\text { Itilineag } \\
\text { ss blas } \\
\text { ve neop } \\
\text { eloproli } \\
\text { absolc } \\
\text { ission; }\end{array}$ & $\begin{array}{l}\text { compare ca } \\
\text { dy with WBC } \\
\text { dysplasia; } \\
\text { 1/2; MDS, } \\
\text { asms; CML, } \\
\text { rative neopl } \\
\text { e neutrophil } \\
D \text {, stable dis }\end{array}$ & & $\begin{array}{l}\text { al variable } \\
\times 10^{9} / \mathrm{L} \text {. } \\
\mathrm{S} \text {, refractor } \\
\text { ysplastic } \\
\mathrm{c} \text { myeloid } \\
\text { nclassifiab } \\
\mathrm{HB} \text {, } \\
\mathrm{PD} \text {, }\end{array}$ & \\
\hline
\end{tabular}




\section{Figure 1}

a

\begin{tabular}{|c|c|c|c|}
\hline Variable & \multicolumn{2}{|c|}{ Odds ratio for $\mathrm{CR}(95 \% \mathrm{Cl})$} & $P$-value \\
\hline Lower HB & $\longrightarrow$ - & $0.25(0.09,0.59)$ & 0.004 \\
\hline Multi-hit TP53 & $1 \rightarrow$ & $3.38(1.74,6.60)$ & $<0.001$ \\
\hline$A S X L 1$ & $\longrightarrow$ & $0.38(0.11,1.03)$ & 0.084 \\
\hline EZH2 & $\longmapsto 1$ & $0.37(0.06,1.32)$ & 0.186 \\
\hline
\end{tabular}

b

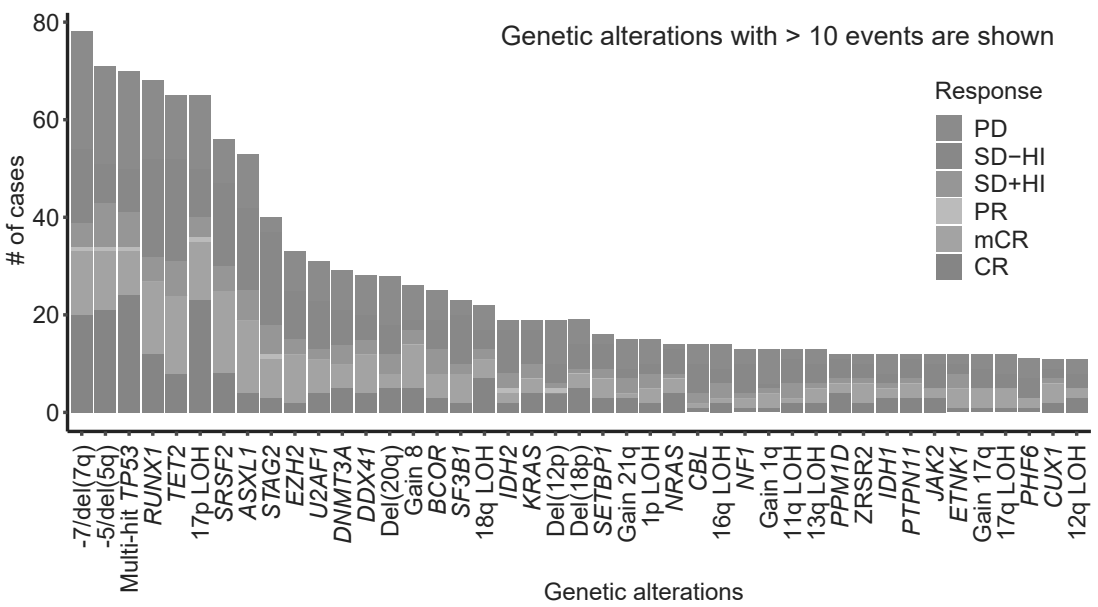

C Discovery cohort by TP53 allelic state

e Discovery cohort according to new risk classes
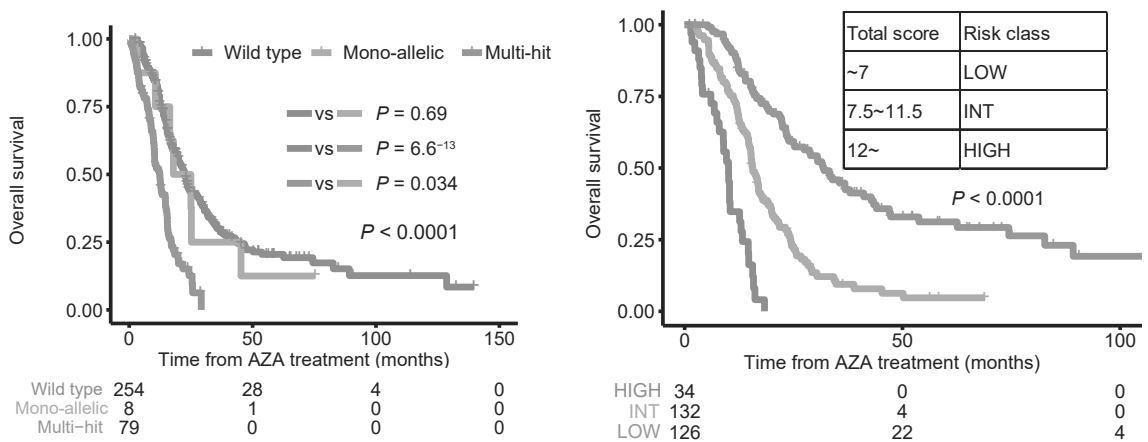

d

\begin{tabular}{|c|c|c|c|c|}
\hline Variable & Hazard ratio for & r OS $(95 \% \mathrm{Cl})$ & $P$-value & Weight \\
\hline IPSS-R raw score & I & $1.32(1.20,1.44)$ & $<0.001$ & 1 \\
\hline Multi-hit TP53 & $1-$ & $1.62(1.10,2.39)$ & 0.01 & 2 \\
\hline$D D \times 41$ & $\longmapsto 1$ & $0.36(0.20,0.65)$ & $<0.001$ & -4 \\
\hline Overall resposne + & 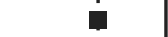 & Reference & & \\
\hline- & 1 & $2.10(1.58,2.80)$ & $<0.001$ & 3 \\
\hline
\end{tabular}

Figure 1 | Genetic and clinical factors associated with response and overall survival.

a, A forest plot showing the result of a multivariate logistic regression analysis for the factors associated with achieving CR performed on the discovery cohort with complete data for response analysis $(n=311)$. Explicative variables are hemoglobin level, mutations in TP53 and ASXL1. Hemoglobin level was dichotomized with a threshold of $80 \mathrm{~g} / \mathrm{L}$; the $\mathrm{x}$-axis is log10 scaled. b, A bar plot showing the number of cases with the response indicated by color that have the genetic alterations indicated on the x-axis. c, Kaplan-Meier estimates of overall survival per TP53 allelic state. The number of the cases at risk at each time is indicated in the tables below. P values are derived from two-sided log-rank tests. d, A forest plot showing the result of Cox proportional hazards regression for overall survival (OS) on the discovery cohort with complete data for OS analysis $(n=341)$. Explicative variables are IPSS-R raw score, multi-hit TP53 mutation, DDX41 mutation, and clinical response (achievement of overall response (OR) or not). The $\mathrm{x}$-axis is log10 scaled. Weight indicates coefficient to construct a novel risk-stratification model based on this multivariate model. Specifically, total score of the novel scoring model is calculated as $1 \times($ IPSS-R raw score $)+2 \times($ Multi-hit TP53 mutation) $-4 \times(D D X 41$ mutation $)+3 \times($ no achievement of OR). e, All the cases in the discovery cohort were stratified into three risk classes (LOW: total score $7 /$ INT: total score $7.5 \sim 11.5 / \mathrm{HIGH}$ : total score 12 ) based on the total score calculated in Fig. 1d. The number of the cases at risk at each time is indicated in the tables below. $P$ values are derived from two-sided log-rank tests.

CR, complete remission; mCR, marrow CR; PR, partial remission; SD, stable disease; HI, hematological improvement; PD, progressive disease; HB, hemoglobin; PLT, platelet; Del, deletion. 
Figure 2

a
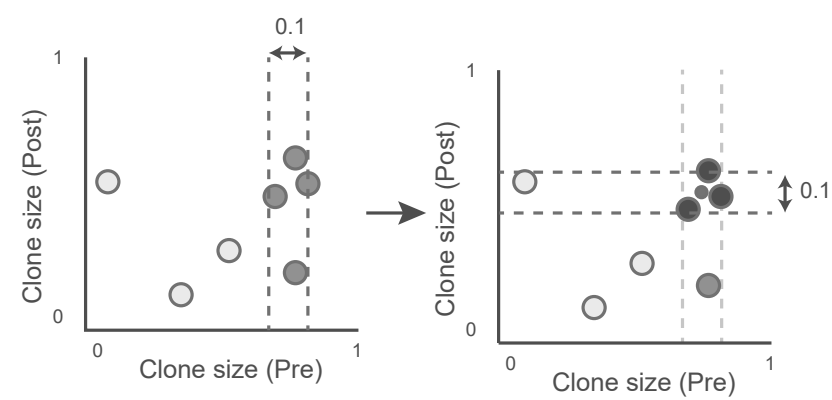

The largest clones in a pre-treatment sample (MC $\left.{ }^{\text {pre }}\right)$

b

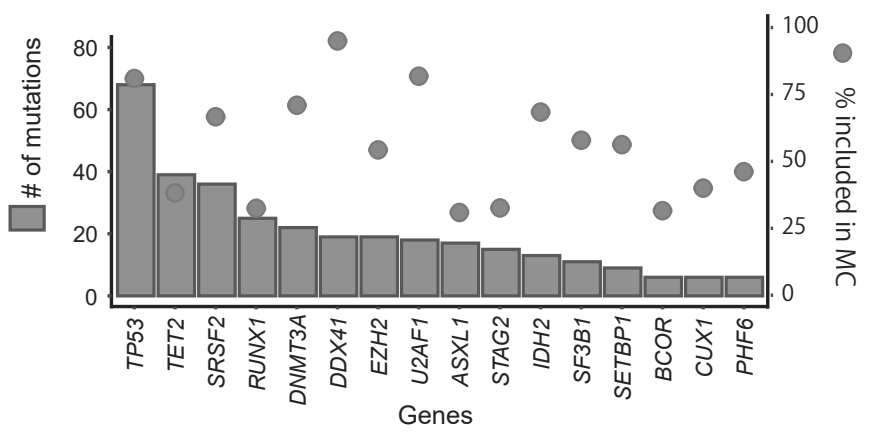

C

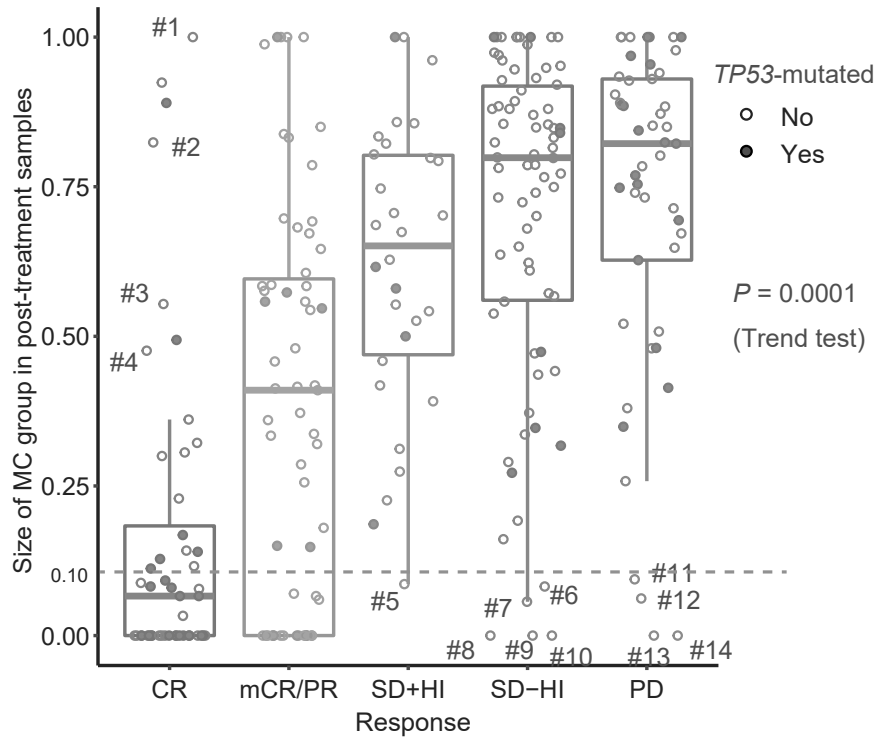

d

UPN-197, 74 y.o., Female, MDS EB2, IPSS-R High

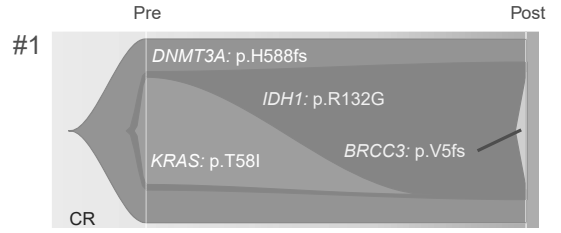

UPN-261, 62 y.o., Male, CMML

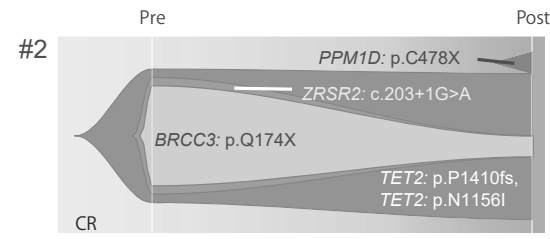

UPN-190, 73 y.o., Female, MDS RCMD, IPSS-R Intermeiate

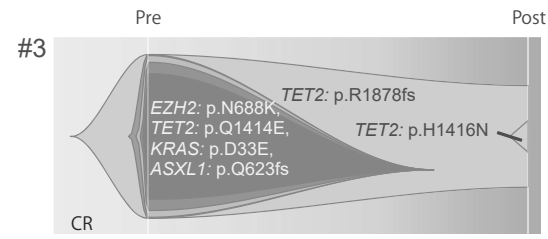

UPN-315, 63 y.o., Male, EB2, IPSS-R Intermeiate

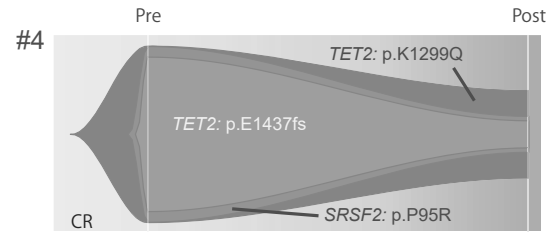

e

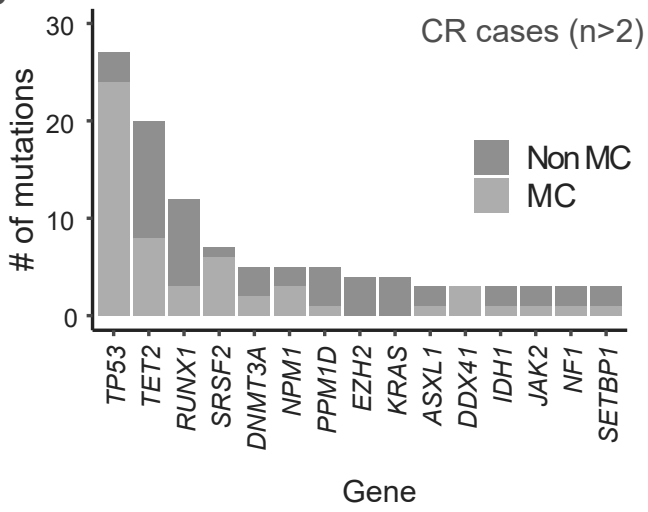

f

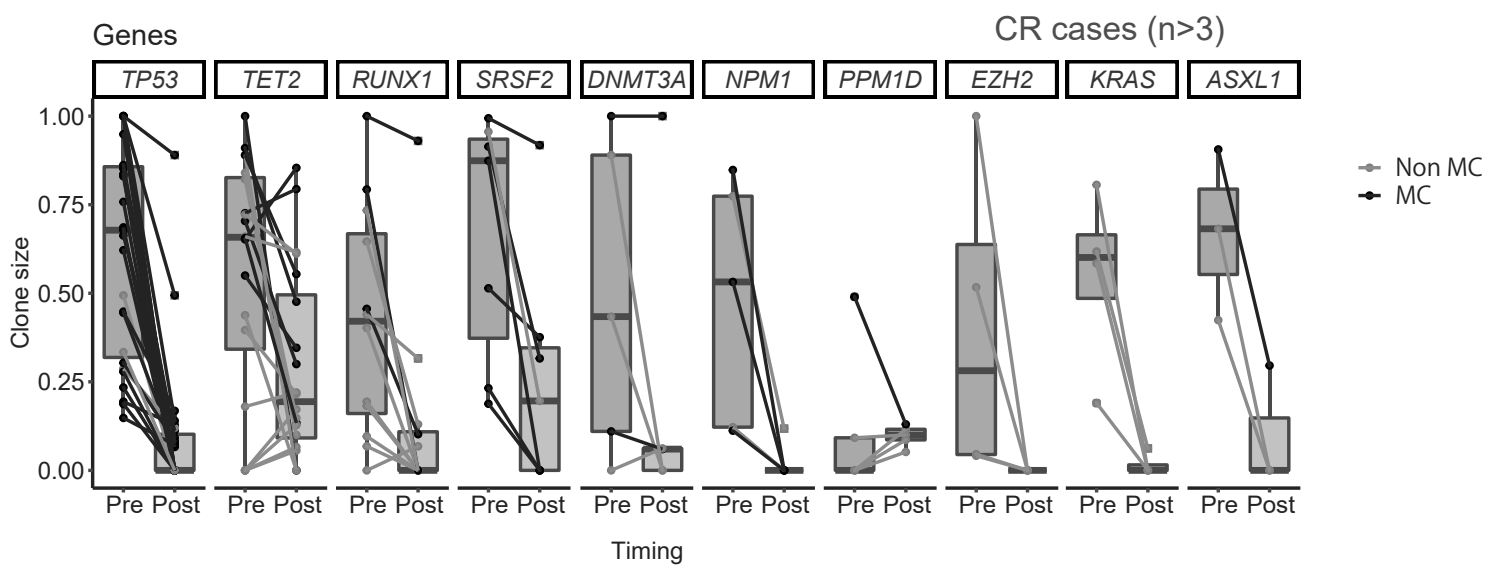


Figure 2 | Clone changes during azacitidine treatment and genetic mechanisms of CR.

a, A schematic explanation of major clones (MC) determined accounting for the clone size in pre- (x-axis) and post-treatment (y-axis) samples from a patient. A set of mutations showing the largest and near largest size (difference in size is $<0.10$ compared with the largest mutation) in a pre-treatment sample were defined as $\mathrm{MC}^{\text {pre }}$ (left panel). Among the MCpre, a set of mutations showing the largest and near largest size in a post-treatment sample were assumed MC (right panel). The detailed description of definition appears in Methods. b. Bar plots showing the numbers of major clones (MC) (left axis) represented by the genes indicated on x-axis. Filled circles indicate the proportion of the variants (right axis) classified as MC out of all the mutations in the paired cohort. c, Box plots showing the MC group sizes of post-treatment samples in the paired cohort having the response indicated on x-axis. $P=0.0001$, Jonckheere-Terpstra tests. d, Fish plots showing the estimated transition of the clone structure during pre- and pot-treatment periods for the $4 \mathrm{CR}$ cases that simultaneously had large MC group sizes (>0.40) and shrinking subclones. Sample IDs correspond to those indicated in Fig. 2c. e, A bar plot showing the number of clones represented by the genes indicated on x-axis. Only clones seen in CR cases are shown. Colors indicate whether each clone was classified as MC or not. f, Paired box plots showing the changes in clone size between pre- and post-treatment samples with mutations in indicated genes. The same mutations are connected by lines. Colors of lines represent whether they are MC or not. 
Figure 3

a

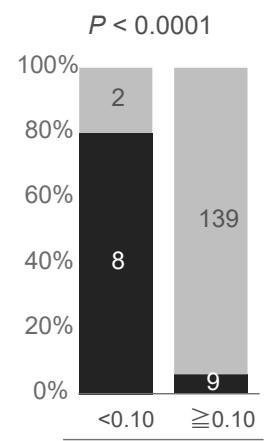

MC group size in SD/PD cases

- DDX41 germline mutation +

DDX41 germline mutation -

\section{b}

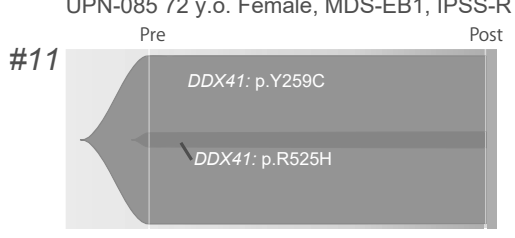

UPN-158 70 y.o. Female, AML-MRC, IPSS-R Intermediate

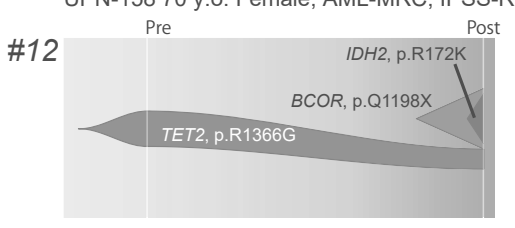

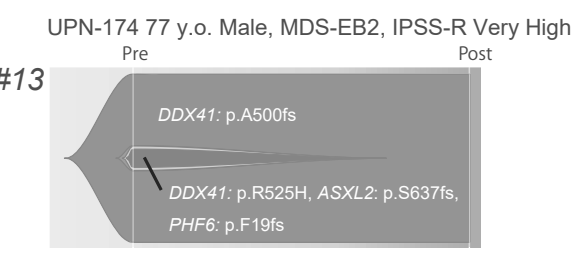

UPN-348 71 y.o. Male, MDS-EB2, IPSS-R High

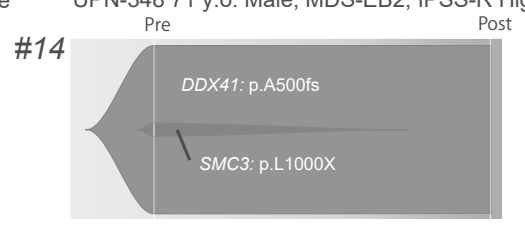

C

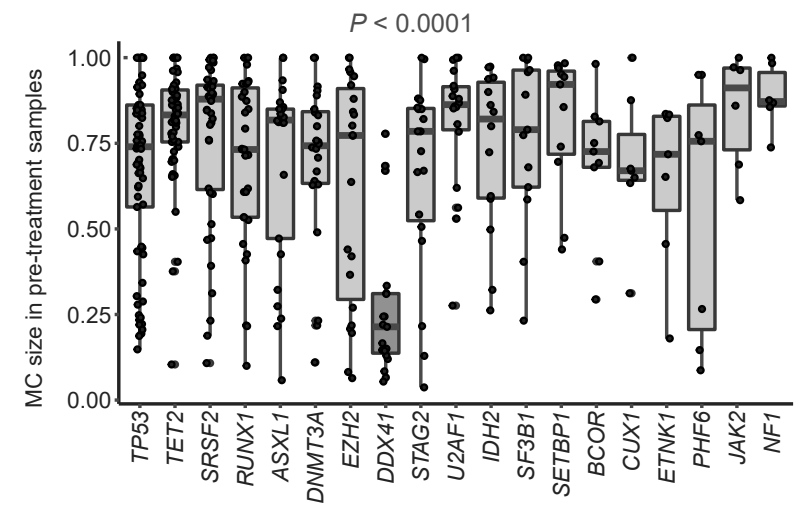

d
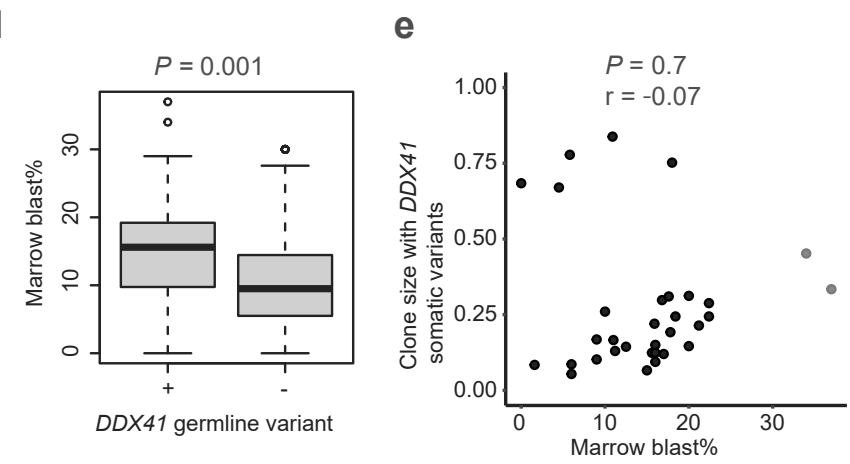

f
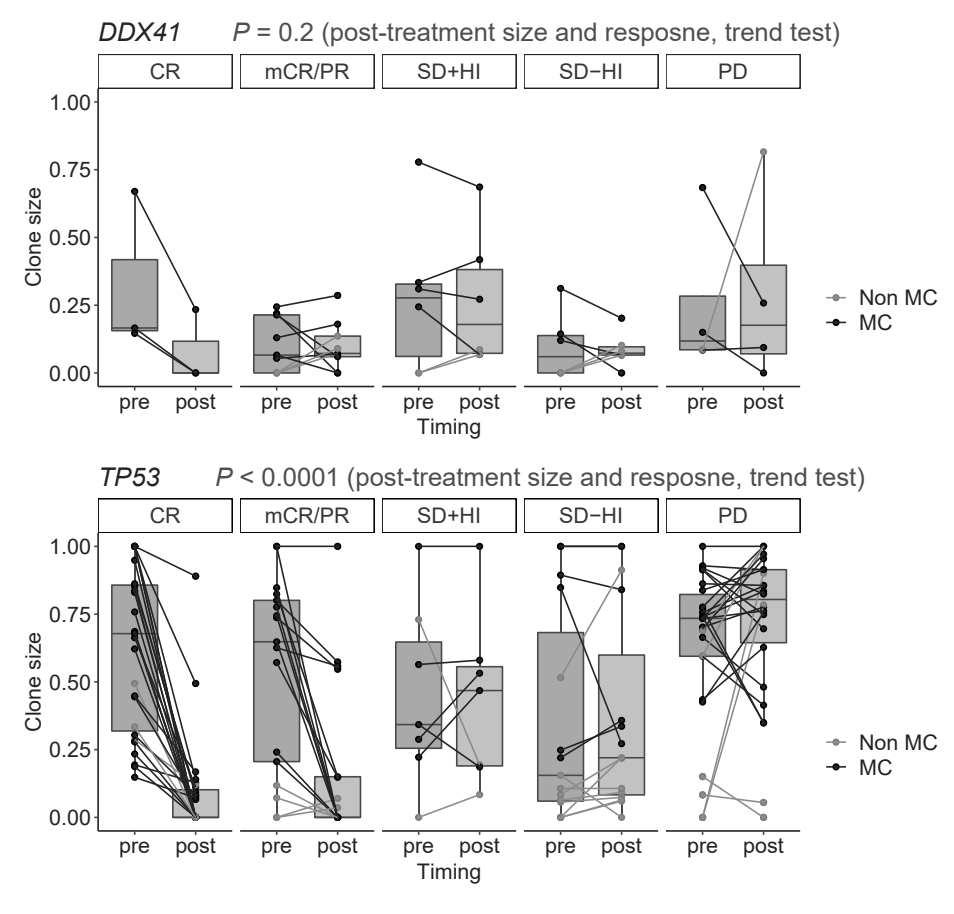

g

OS by post-treatment $D D X 41$ clone size

$$
\perp \leq \text { median } \perp>\text { median }
$$

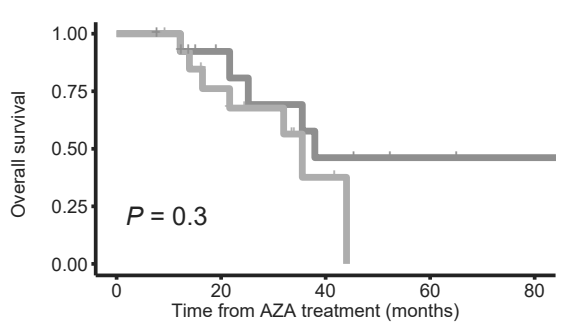

Number at risk
OS by post-treatment TP53 clone size

$$
\perp \leq \text { median } \perp \text { median }
$$

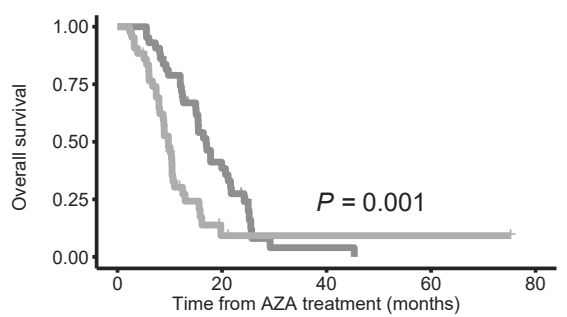

Number at risk 
Figure 3 | Somatic DDX41-mutated clone size does not correlate with blast ratio or response to treatment.

a, Percentage of the cases that have DDX41 germline variants is shown among the cases with stable diseases (SD) or progressive disease (PD) having major clone (MC) group sizes $<0.10(n=10)$ and $>=0.10(n=148)$. $P=1.22 \times 10^{-7}$, Fisher' $\mathrm{s}$ exact test. b. Fish plots showing estimated transition of the clone structure throughout the pre- and pot-treatment periods for the 4 PD cases that had post-treatment MC group sizes $<0.10$. Sample IDs corresponds to those indicated in Fig. 2 c. c, Box plots showing the size of major clone (MC) groups in pre-treatment samples that have mutations in the genes indicated on $\mathrm{x}$-axis. $\mathrm{A}$ box plot representing DDX41-mutated MC groups is highlighted with red color. $P$ value is derived from a two-sided t-test for comparison of pre-treatment MC sizes represented by DDX41 and the other genes. d, Box plots showing marrow blast percentage for the cases with or without $D D X 41$ germline mutations. $P$ value is derived from a two-sided t-test. e, A scatter plot showing marrow blast percentage (x-axis) and size of clones represented by somatic DDX41 mutations ( $\mathrm{y}$-axis). $P$ value is derived from a Pearson' s correlation test and $r$ value indicates Pearson' s product moment correlation coefficient. f, Paired box plots showing the changes in clone size between pre- and post-treatment samples with mutations in DDX41 (upper panel) and TP53 (lower panel). The same mutations are connected by lines. Colors of lines represent whether they are MC or not. $P$ values are derived from Jonckheere-Terpstra tests under the hypothesis that there is no correlation between clinical response and post-treatment clone sizes. g, Kaplan-Meier estimates of overall survival (OS) per clone sizes in post-treatment samples. Cases with somatic DDX41 mutations (left, $n=28$ ) and TP53 mutations (right, $n=68$ ) are shown. The clone sizes were divided by the median value for each gene. The number of the cases at risk at each time is indicated in the table below. $P$ values are derived from two-sided log-rank tests. 


\section{Figure 4}

a

Profile of NEC ( $n>4)$

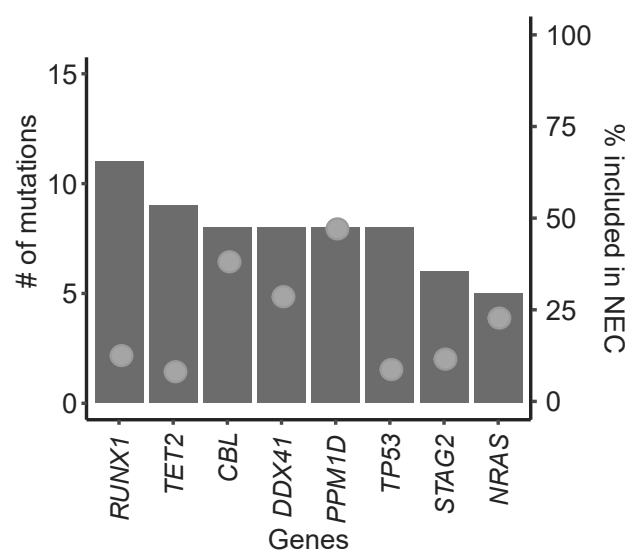

C

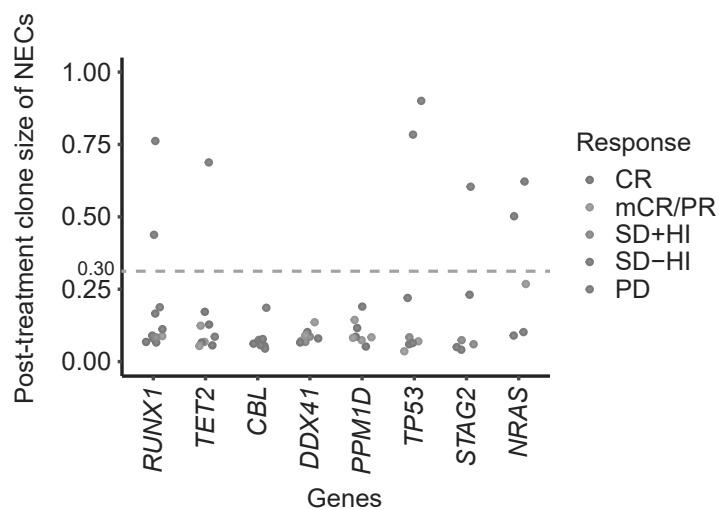

e

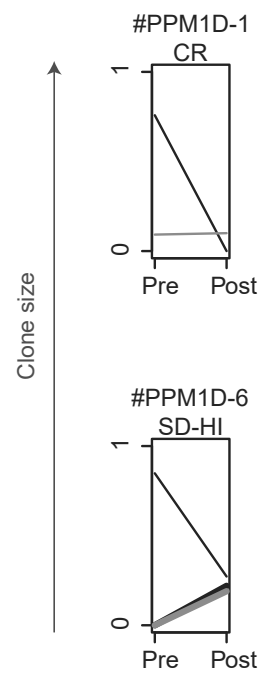

b

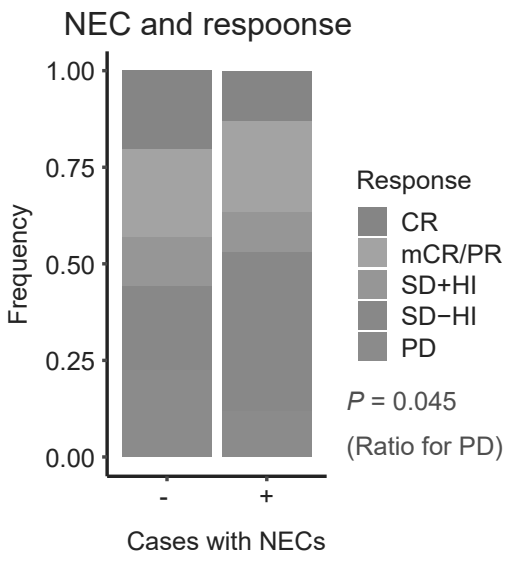

d

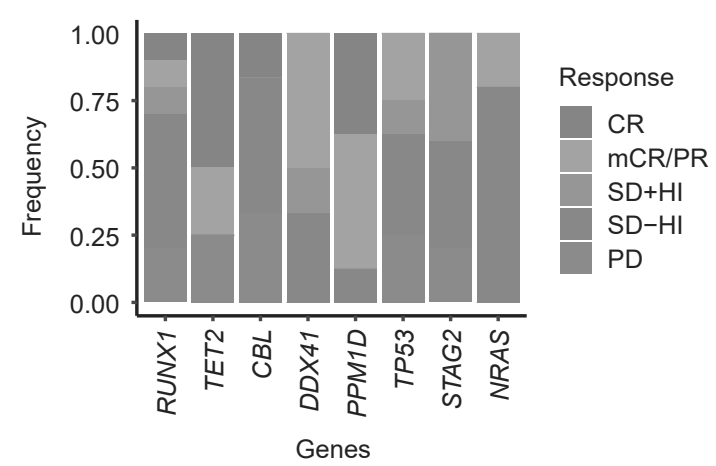

f

Case \#PPM1D-1,

Pre-treatment sample
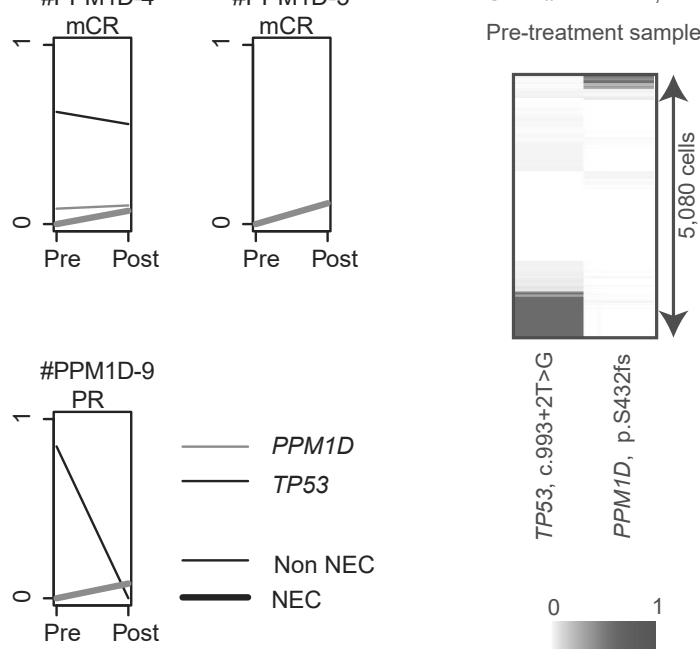

Allele frequency

Figure 4 | Property and role of newly emerging clones (NECs) after azacitidine treatment.

a, Bar plots showing the numbers of newly emerging clones (NEC) (left axis) represented by the genes indicated on x-axis. Filled circles indicate the proportion of the mutations (right axis) classified as NEC out of all the mutations in the paired cohort. $\mathbf{b}$, Bar graphs showing the proportion of the cases with or without NEC showing responses indicated by color. $\mathrm{P}$ value is derived from two-sided Fisher' s exact test to estimate the frequency of the cases having progressive disease (PD). c, Jitter plots showing the post-treatment clone size of NECs represented by the genes indicated on x-axis. Colors indicate clinical response. d, Bar graphs showing the proportion of the cases with NEC represented by the genes indicated on $x$-axis having the response indicated by colors. e, Clone size transition in pre- and post-treatment samples of TP53 (black) and PPM1D (red) in the 9 cases having mutations in PPM1D classified as DECs. Bold lines indicate variant classified as DECs. f, Single cell analysis of the case \#PPM1D-1 showing variant allele frequency of mutations in TP53 and PPM1D in 5,080 cells. Shaded colors indicate the variant allele frequencies of the variants. 
Figure 5

a

\begin{tabular}{|c|c|c|c|c|c|}
\hline \multirow{2}{*}{\begin{tabular}{|l} 
Variable \\
IPSS-R score
\end{tabular}} & \multirow{2}{*}{$\frac{N}{267}$} & \multicolumn{2}{|c|}{ Hazard ratio for OS $(95 \% \mathrm{Cl})$} & \multirow{2}{*}{$\begin{array}{l}P \text {-value } \\
<0.001\end{array}$} & \multirow{2}{*}{$\frac{\text { Weight }}{1}$} \\
\hline & & in & $1.33(1.20,1.47)$ & & \\
\hline Multi-hit TP53 & 267 & $1 \mapsto$ & $1.97(1.27,3.04)$ & 0.002 & 2 \\
\hline DDX41 & 267 & $\longmapsto \longrightarrow 1$ & $0.41(0.20,0.81)$ & 0.011 & -3 \\
\hline MaxVAF post & 267 & $\mapsto$ & $2.16(1.28,3.66)$ & 0.004 & 3 \\
\hline Overall response & +130 & 1 & Reference & & \\
\hline & 137 & $\rightarrow-1$ & $1.47(1.04,2.09)$ & 0.030 & 1 \\
\hline
\end{tabular}

C

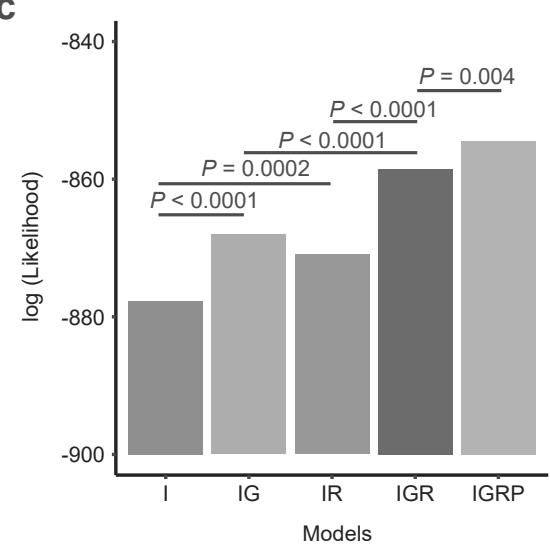

b

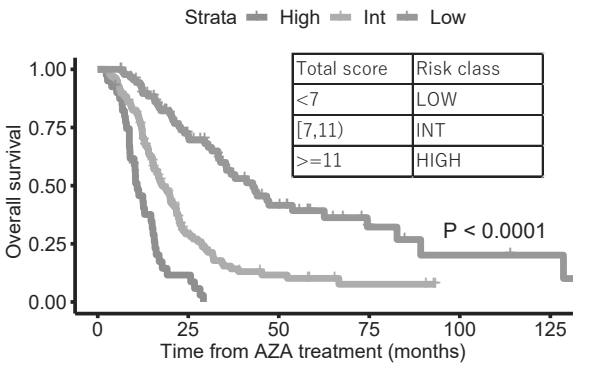

Number at risk

$\begin{array}{ccccccc}\text { High } & 40 & 4 & 0 & 0 & 0 & 0 \\ \text { Low } 136 & 27 & 8 & 3 & 0 & 0 \\ \text { Low } & 91 & 50 & 21 & 7 & 3 & 2\end{array}$

e

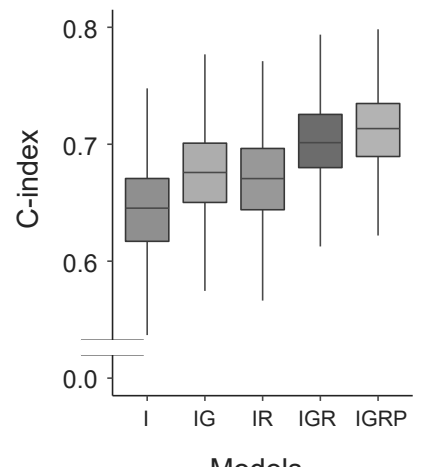

f

$\underline{T P 53}$ Clone size

홍 范

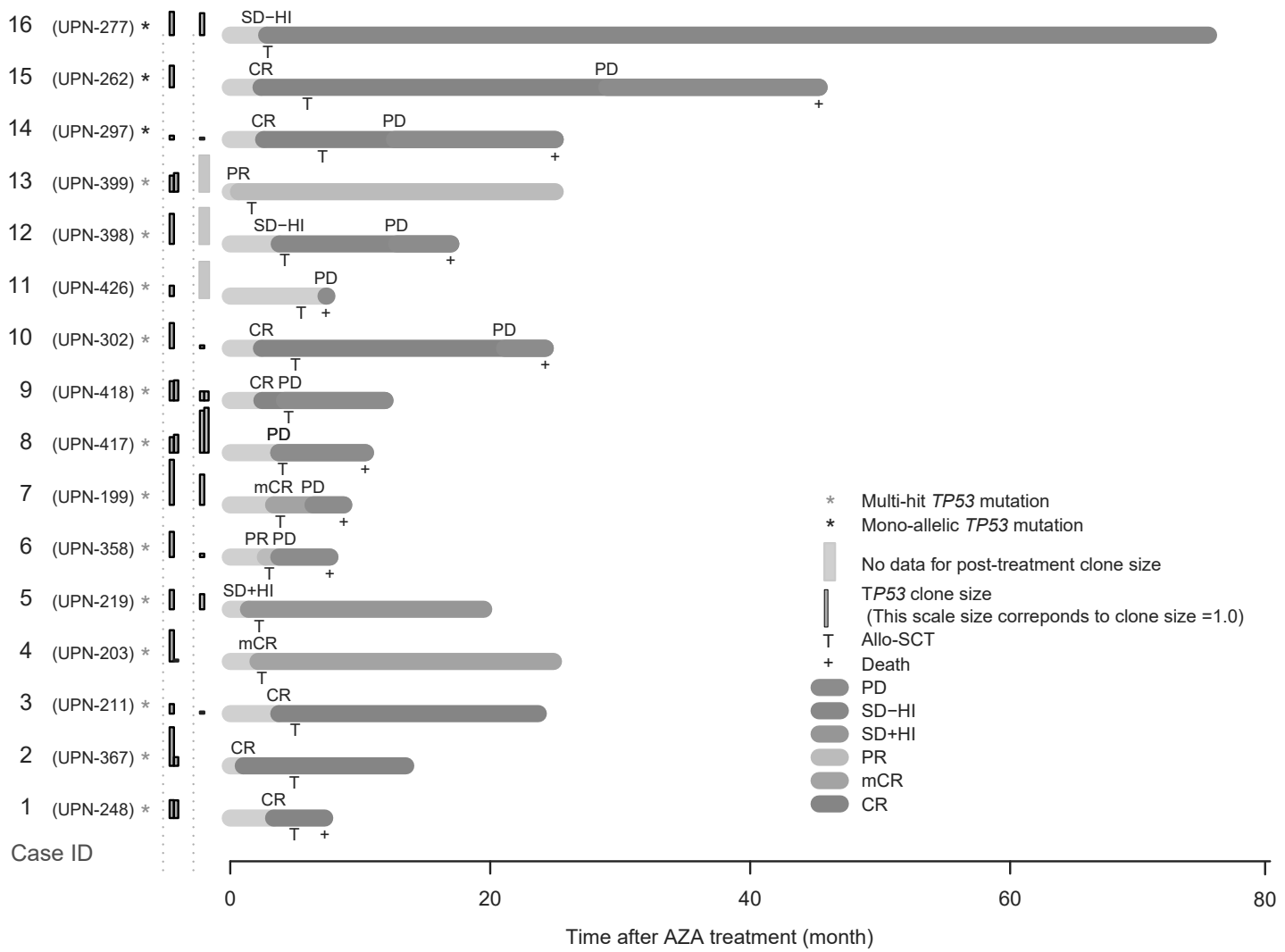


Figure 5 | The role of post-treatment clone size on improvement of OS model and predicting post-transplant outcomes. a, A forest plot showing the result of Cox proportional hazards regression for overall survival (OS) performed on the paired cohort with complete data for OS analysis $(n=267)$. N indicates the number of cases having the variables. Explicative variables are International Prognostic Scoring System Revised (IPSS-R) raw score, pre-treatment mutations (multi-hit TP53 mutations, DDX41 mutations), the largest variant allele frequency (VAF) values adjusted for copy number alterations in post-treatment samples (MaxVAF ${ }^{\text {post }}$ ), and overall response according to the International Working Group (IWG) 2006 criteria. Overall response includes complete remission (CR), marrow $\mathrm{CR}(\mathrm{mCR})$ and partial remission (PR); the x-axis is log10 scaled. Weight indicates coefficient to construct a novel risk-stratification model based on this multivariate model. Specifically, total score of the novel model is calculated as $1 \times($ IPSS-R raw score $)+2 \times($ Multi-hit TP53 mutation $)-3 \times(D D X 41$ mutation $)+3 \times($ MaxVAFpost value $)+1 \times$ (no achievement of OR). b, All the cases were stratified into three risk classes (LOW: total score $\sim 7 / \mathrm{INT}$ : total score 7 11/HIGH: total score 11 ) based on the total score calculated in Fig. 5a. Kaplan-Meier estimates of overall survival per risk classes of the proposed model is presented. The number of the cases at risk at each time is indicated in the tables below. $P$ values are derived from two-sided log-rank tests. c, Bar graphs showing log-converted likelihood of Cox proportional hazards regression for overall survival (OS) models including the explicative variables symbolized by the four capital letters. Each of the symbols represents I, IPSS-R raw score; G, pre-treatment gene mutations in which multi-hit TP53 and DDX41 mutations are included; R, overall response; $P$, the largest variant allele frequency (VAF) values adjusted for copy number alterations in post-treatment samples. d, A schematic presentation of the analysis that examines the improvement of predictability. The paired cohort was randomly split into $75 \%$ training and $25 \%$ validation subsets 10,000 times and constructed a model with each of the training set and fit the model and calculated the concordance index (c-index) for the corresponding validation set. e, Box plots indicating the distribution of c-index in the validation cohorts of 10,000 simulations. The symbols that represent models are the same as in Fig. 5c. f, A swimmer' s plot showing the clinical course for the 16 transplanted cases with TP53 mutations. Red and black asterisks indicate TP53 allelic state. Colors of horizontal bars indicate the period having the clinical state. Vertical bars show the clone size of TP53 variants in pre- and post-treatment samples. CR, complete remission; mCR, marrow CR; PR, partial remission; SD, stable disease; HI, hematological improvement; PD, progressive disease. 


\section{Extended Data Figure 1}

a
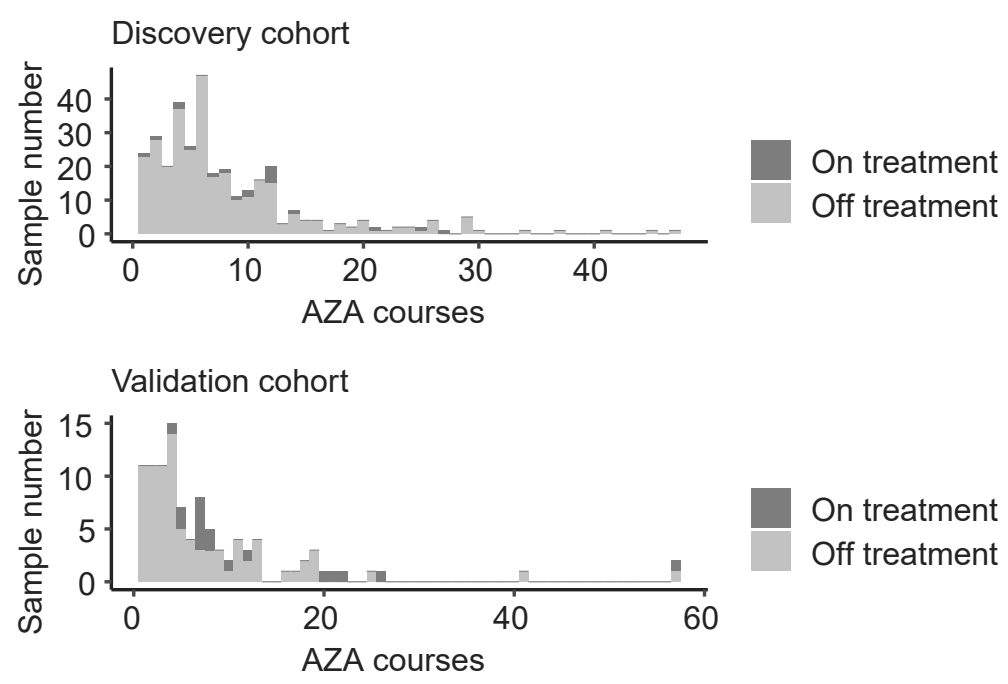

b

OS in discovery and validation cohort

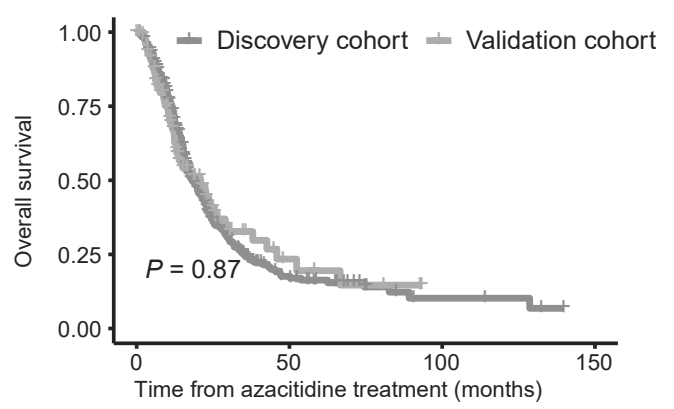

$\begin{array}{lccc}\text { Discovery cohort } 341 & 29 & 4 & 0 \\ \text { Validation cohort } 110 & 6 & 0 & 0\end{array}$

\section{C}

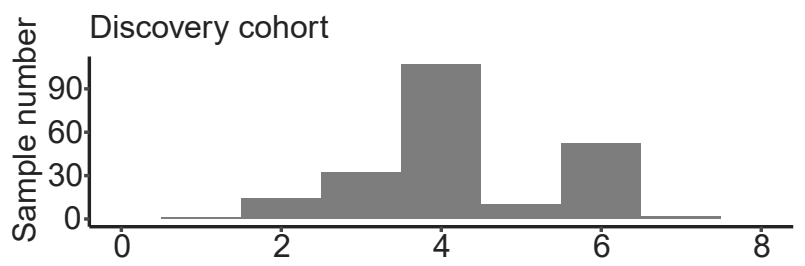

Azacitidine courses of post-treatment sample collection

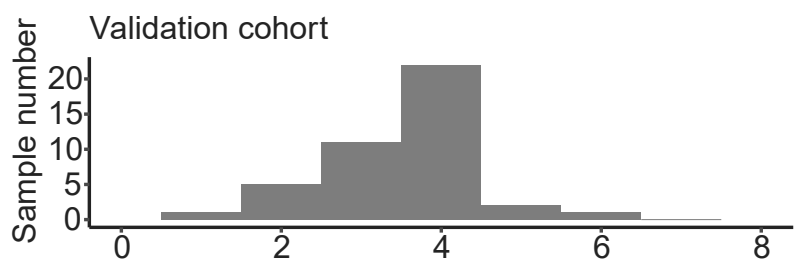

Azacitidine courses of post-treatment sample collection

Extended Data Figure 1 | Azacitidine treatment profile, overall survival and time to response.

a, Histograms showing the number of all azacitidine treatment courses for the discovery (upper panel, $n=336$ ) and the validation (lower panel, $n=101$ ) cohorts with complete data for analysis. Colors indicate whether the cases are still on azacitidine treatment or not. b. Kaplan-Meier estimates of overall survival (OS) on 341 cases in the discovery cohort and 110 cases in the validation cohort. The numbers of the cases at risk at each time are indicated in the tables below. $P$ value is derived from a two-sided log-rank test. c. Histograms showing the number of azacitidine treatment courses when post-treatment samples were collected for the discovery (upper panel, $n=265$ ) and the validation (lower panel, $n=68$ ) cohorts. The sampling time was fixed as after course 4 for JALSG-MDS-212 cohorts, while it was not fixed in the other cohorts. 


\section{Extended Data Figure 2}

a

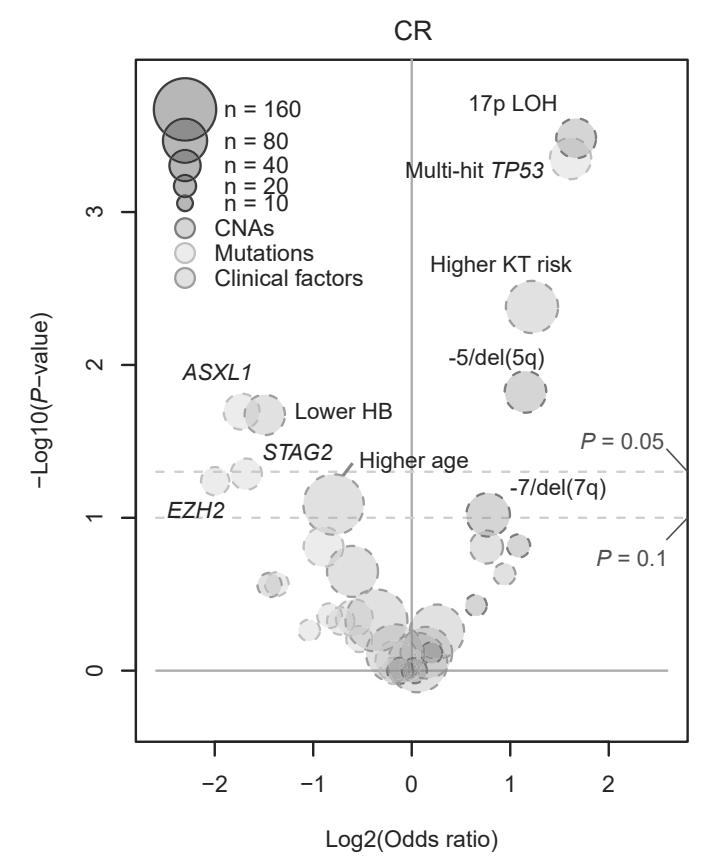

d

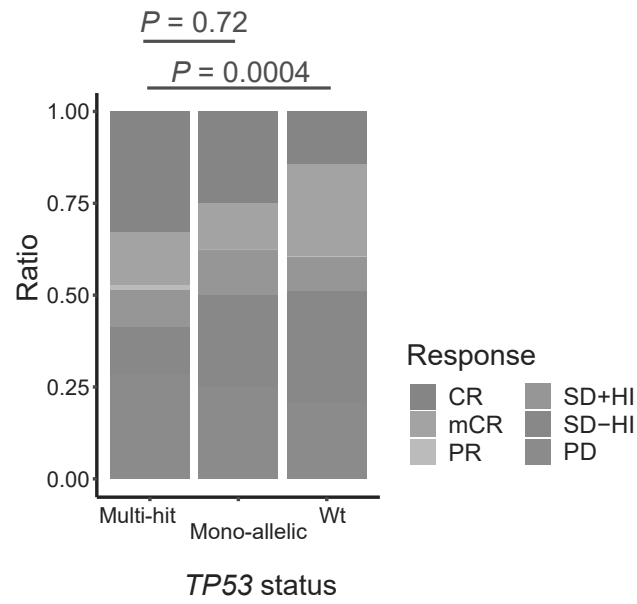

b

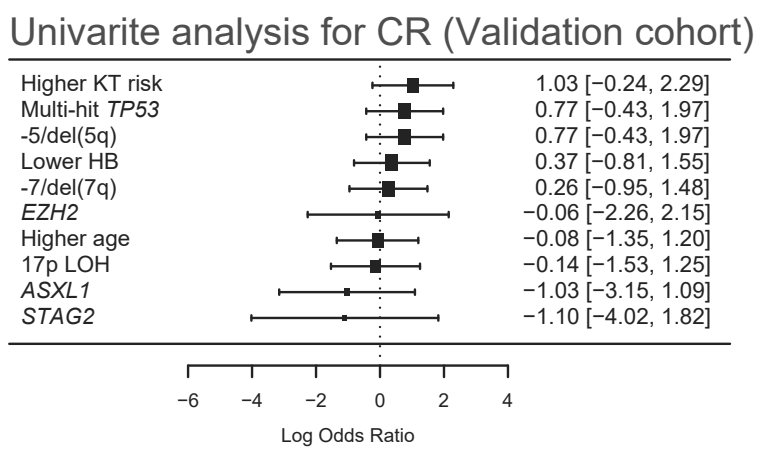

C

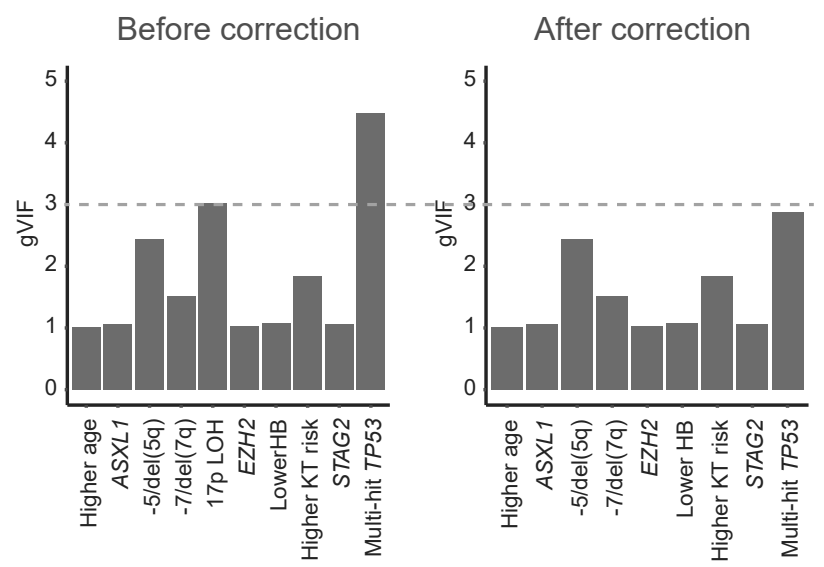

Extended Data Figure 2 | Factors associated with achieving complete remission (CR).

a, A volcano plot showing the result of univariate analyses for the factors associated with achieving CR. X-and $y$-axis show odds ratio and $P$-values derived from two-sided Fisher' s exact tests. The $\mathrm{x}$ - and y-axis are log 2 and log 10 scaled, respectively. Area of circles indicates the number of samples having the features indicated by the covariates and color of circles indicates the attribute of the covariates. Dotted lines indicate significant $(P=0.05)$ and sub-significant $(P=0.10)$ levels. Hemoglobin level (HB) was dichotomized with a threshold of $80 \mathrm{~g} / \mathrm{L}$ and karyotype (KT) risks were classified into higher (poor and very poor, according to IPSS-R-based karyotype risk classification) and lower (intermediate, good and very good) groups. b, A forest plot showing the result of univariate analyses for the factors associated with achieving CR for the validation cohort with complete data for response analysis $(n=90)$. Explicative variables are limited to those showing significant $(P<0.05)$ or sub-significant $(P$ $<0.10$ ) association with achieving CR in univariate analyses for the discovery cohort (Extended Data Fig. 2a); $\mathrm{x}$-axis is log10 scaled. c, Bar plots showing generalized variance inflation factor (gVIF) of the covariates indicated on $x$-axis in the multivariate logistic regression model for achieving CR. Left and right panels show gVIF profiles before and after correction for multiple collinearity, respectively (Methods). The variables after the correction (right panel) were subjected to multivariate analysis followed by AIC-based covariate selection. d, Bar plots showing the proportion of cases having the response indicated by color with the TP53 allelic status indicated on the $x$-axis. 


\section{Extended Data Figure 3}

a

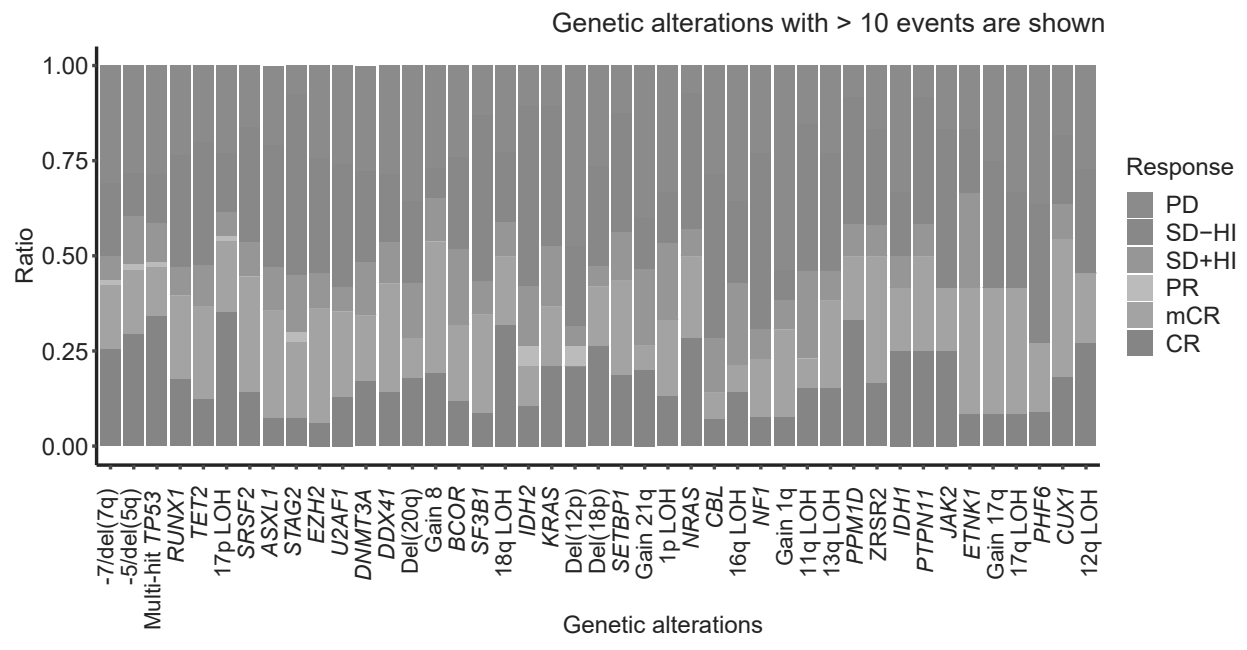

b

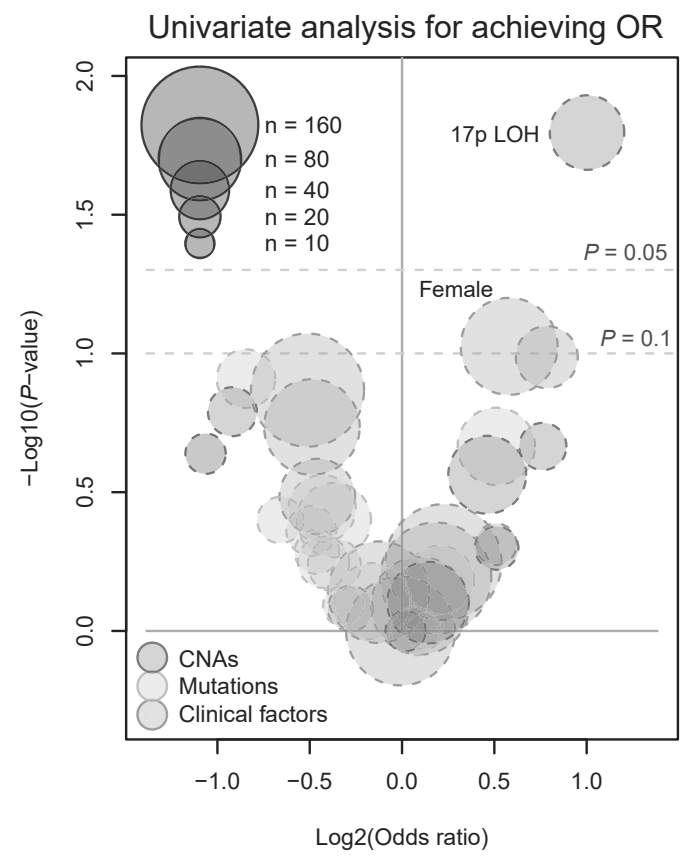

C

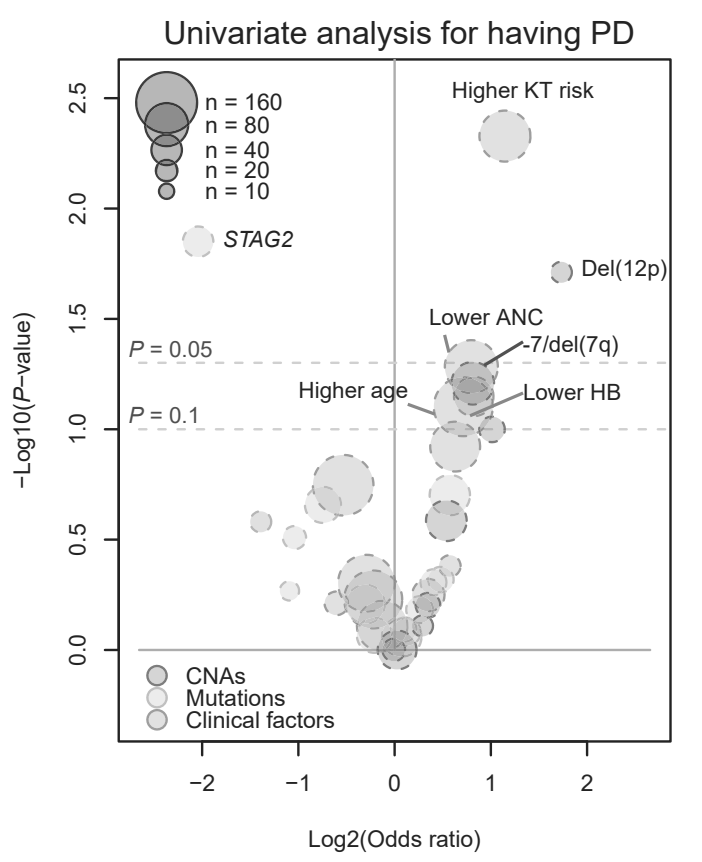

d

\begin{tabular}{|c|c|c|c|}
\hline Variable & \multicolumn{2}{|c|}{ Odds ratio for PD $(95 \% \mathrm{Cl})$} & $P$-value \\
\hline Higher age & त一 & $1.73(1.00,3.02)$ & 0.05 \\
\hline Lower ANC & 㟔 & $1.54(0.88,2.67)$ & 0.13 \\
\hline Higher $\mathrm{KT}$ risk & 드- & $1.80(1.02,3.16)$ & 0.04 \\
\hline STAG2 & $\longrightarrow$ & $0.28(0.07,0.85)$ & 0.05 \\
\hline
\end{tabular}

\section{Extended Data Figure 3 | Factors associated with achieving overall response (OR) and having progressive disease (PD).}

a, Bar plots showing the proportion of cases having the response indicated by color with the genetic alterations indicated on the $\mathrm{x}$-axis. b,c, A volcano plot showing the result of univariate analyses for the factors associated with achieving OR (b) and having PD (c). X-and y-axis show odds ratio and $P$ values derived from two-sided Fisher' s exact tests. The $\mathrm{x}$ - and $\mathrm{y}$-axis are log2 and $\log 10$ scaled. Area of circles indicates the number of samples having the features indicated by the covariates and color of circles indicates the attributes of the covariates. Dotted lines indicate significant $(P=0.05)$ and sub-significant $(P=0.10)$ levels. Hemoglobin (HB) level and absolute neutrophil counts (ANC) were dichotomized with a threshold of $80 \mathrm{~g} / \mathrm{L}$ and $8.0 \times 10^{9} / \mathrm{L}$, respectively. Karyotype (KT) risks were classified into higher (poor and very poor, according to IPSS-R-based karyotype risk classification) and lower (intermediate, good and very good) groups. d, A forest plot showing the result of a multivariate logistic regression analysis for the factors associated with having PD performed on the discovery cohort with complete data for response analysis $(n=311)$. 


\section{Extended Data Figure 4}

a
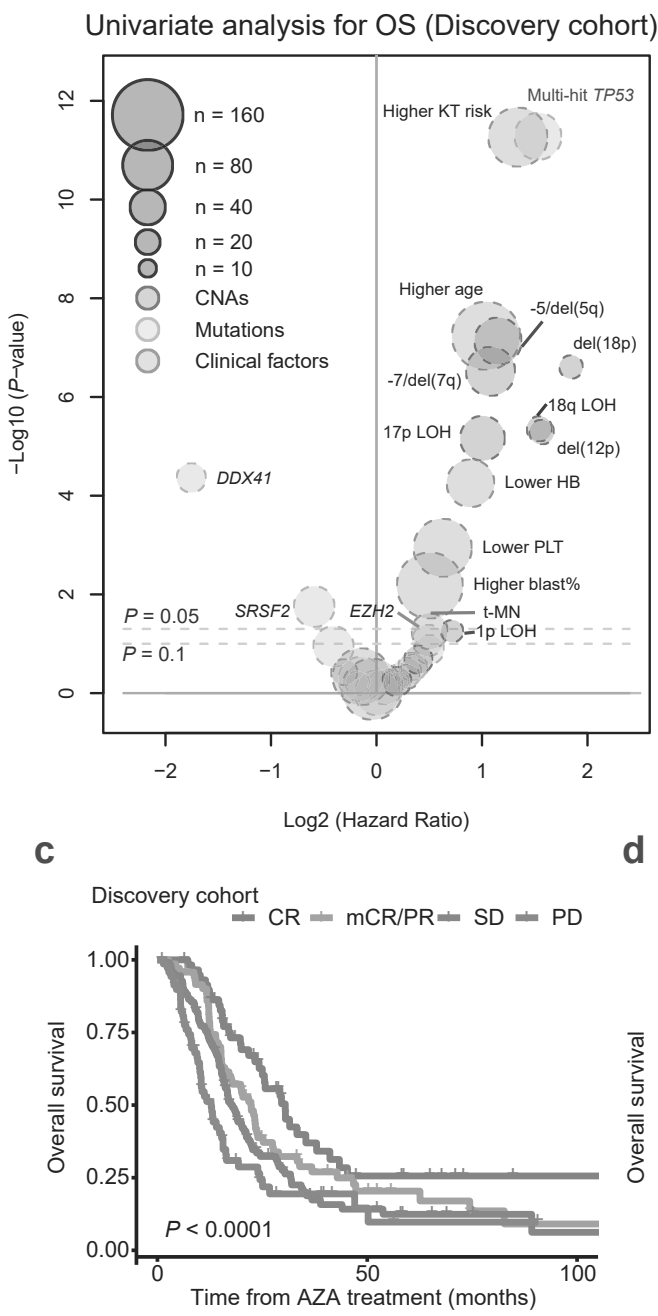
CR 59
mCR/PR 71

SD
PD 710

f

Discovery cohort, CR cases

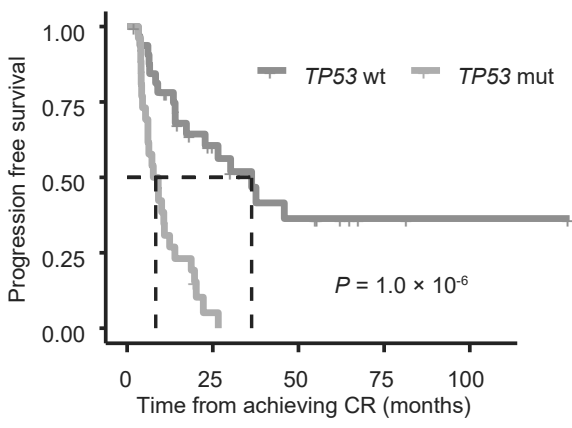

b

Univariate analysis for OS (Validation cohort)

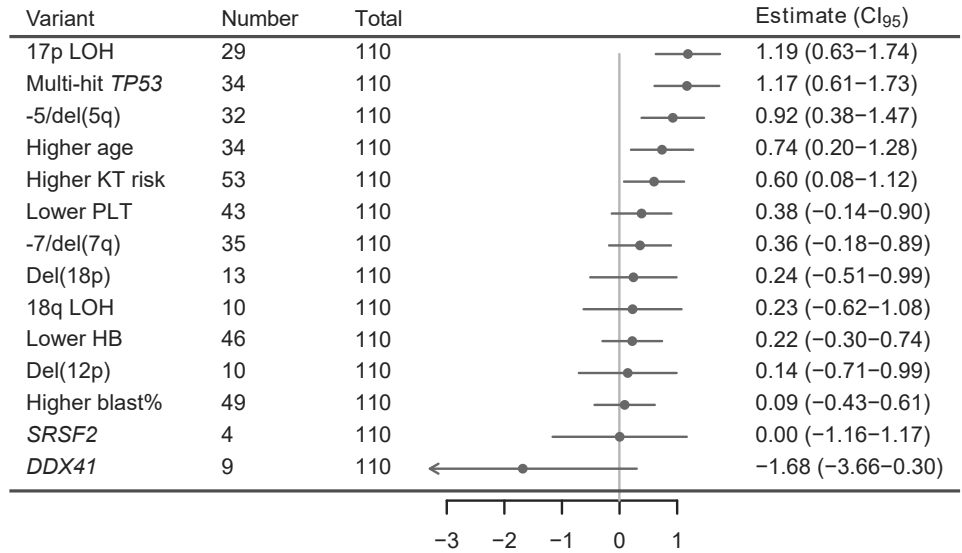

d

Discovery cohort, Multihit TP53-mutated

\lrcorner $\mathrm{CR} \rightleftharpoons \mathrm{mCR} / \mathrm{PR} \doteq \mathrm{SD} \rightleftharpoons \mathrm{PD}$

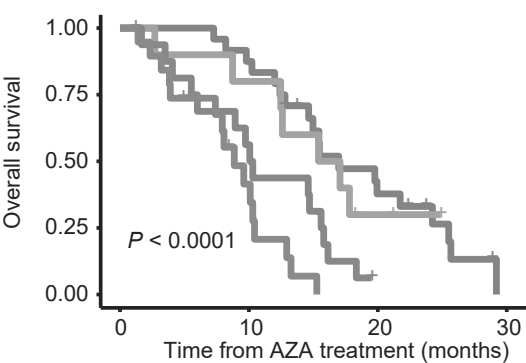

$\begin{array}{rr}\text { CR } & 24 \\ \mathrm{mCR} / \mathrm{PR} & 10 \\ \mathrm{SD} & 16 \\ \mathrm{PD} & 20\end{array}$
21
8
9
6

8
2
0
0

e

Discovery cohort, IPSS-R very high

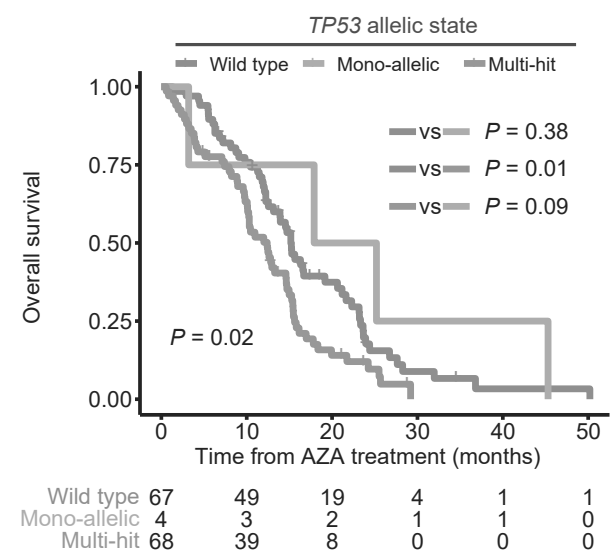

\section{Discovery cohort}

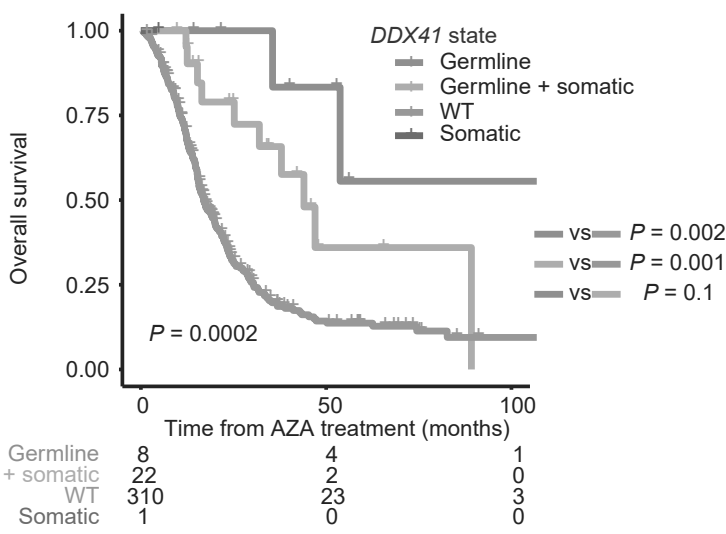

h
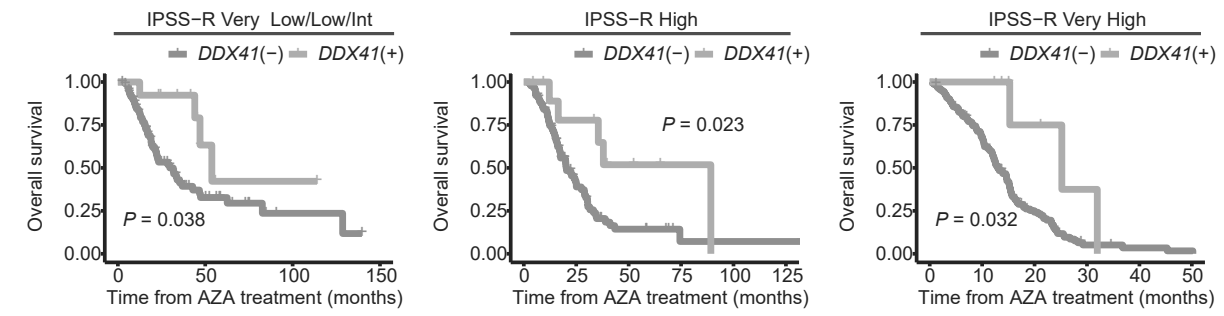

$\begin{array}{llll}D D X 41(-) 88 & 15 & 2 & 0\end{array}$

$\begin{array}{cccccccccccc}D D X 41(-) 89 & 26 & 7 & 1 & 1 & 1 & D D X 41(-) 132 & 84 & 26 & 4 & 2 & 1 \\ D D X 41(+) 11 & 7 & 3 & 1 & 0 & 0 & D D X 41(+) 7 & 7 & 3 & 1 & 0 & 0\end{array}$

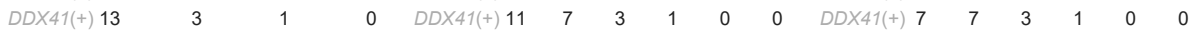

i

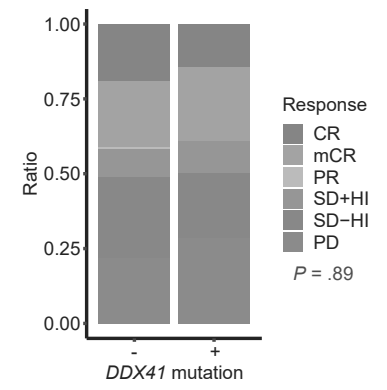


Extended Data Figure 4 | Univariate analysis for overall survival (OS).

a, A volcano plot showing the result of univariate analyses for the factors associated with OS. X-and $y$-axis show hazard ratio and P-values derived from Cox proportional hazards model. The $x$ - and y-axis are log 2 and log10 scaled. Area of circles indicates the number of samples having the features indicated by the covariates and color of circles indicates the attributes of the covariates. Dotted lines indicate significant $(P=0.05)$ and sub-significant $(P=0.10)$ levels. Age, hemoglobin level $(H B)$, platelet level $(P L T)$, marrow blast ratio (blast\%) were dichotomized with a threshold of 72 -year-old, $80 \mathrm{~g} / \mathrm{L}, 50 \times 109 / \mathrm{L}, 10 \%$, respectively. Karyotype (KT) risks were classified into higher (poor and very poor, according to IPSS-R-based karyotype risk classification) and lower (intermediate, good and very good) groups. b, A forest plot showing the result of univariate analysis for the factors associated with OS performed on the validation cohort with complete data for survival analysis $(n=110)$. Explicative variables are limited to those showing significance $(P<0.05)$ in univariate analysis in the discovery cohort (Extended Data Fig. 4a); $x$-axis is log10 scaled. c,d, Kaplan-Meier estimates of OS per clinical responses on the 311 cases in the entire discovery cohort (c) and on the 70 cases in the discovery cohort having multi-hit TP53 mutations (d) with complete data for the analyses. The numbers of the cases at risk at each time are indicated in the tables below. P-value is derived from a two-sided log-rank test. e, Kaplan-Meier estimates of OS per TP53 allelic state on the 139 cases in the discovery cohort with IPSS-R very high risk. The numbers of the cases at risk at each time are indicated in the table below. P-values are derived from two-sided log-rank tests. f, Kaplan-Meier estimates of progression free survival per TP53 mutation status on the 59 cases who achieved CR. The numbers of the cases at risk at each time are indicated in the table below. $P$ value is derived from a two-sided log-rank test. g, Kaplan-Meier estimates of OS per DDX41 mutational state on the 341 cases in the discovery cohort. The numbers of the cases at risk at each time are indicated in the table below. $P$ values are derived from two-sided log-rank tests. $h$, Kaplan-Meier estimates of overall survival per DDX41 mutation status for IPSS-R very low/low/intermediate (left), high (middle) and very high (right) risk cases. The number of the cases at risk at each time is indicated in the tables below. P values are derived from two-sided log-rank tests. i, Bar plots showing the proportion of the cases having responses indicated by color in DDX41-mutated and unmutated cases. P value is derived from a Fisher' s exact test. 


\section{Extended Data Figure 5}

a

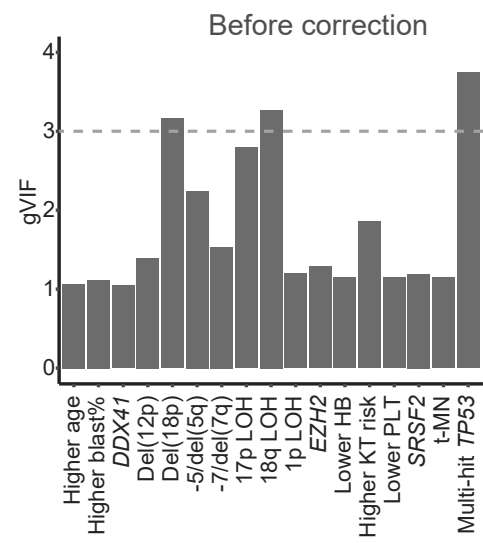

b

After correction

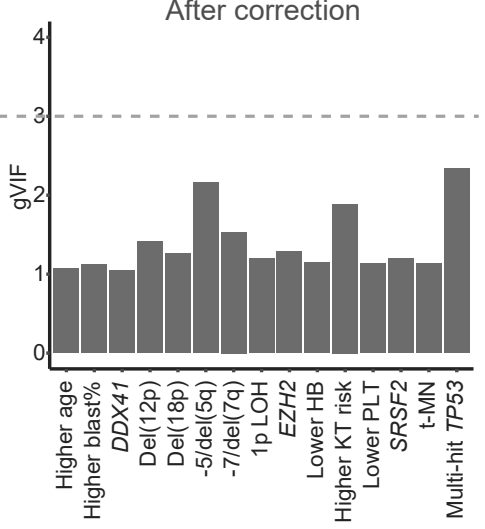

C

Discovery cohort by IPSS-R

- Very low/Low/Intermediate - High + Very high

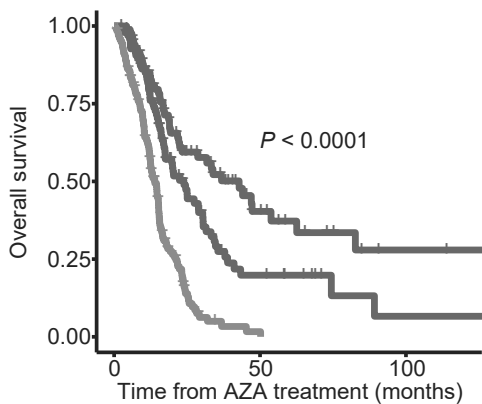

Very low/Low/Intermediate 85

very high 139

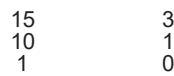

e

Discovery cohort with IPSS-R: VeryHigh
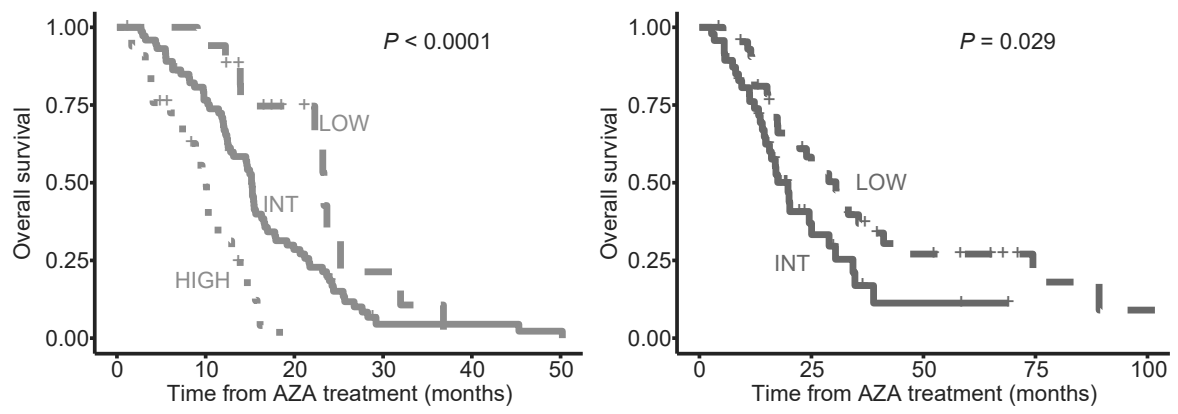

HIGH 34

INT 73
LOW 17

15
55
16

15
55
16

0
20
8

0
2
0

0
1
0

INT 47

LOW 44

Discovery cohort with IPSS-R: High

d

Cohort

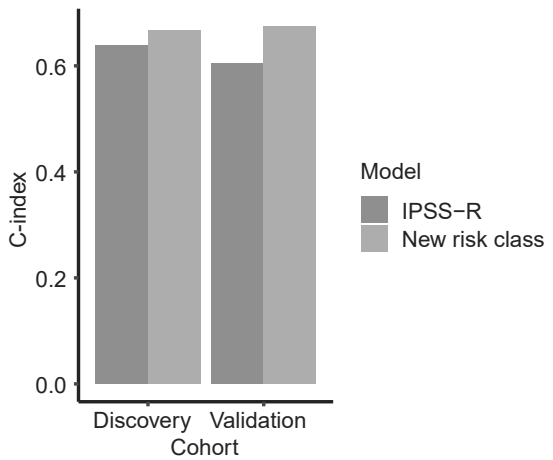

\begin{tabular}{|c|c|c|c|}
\hline Variable & \multicolumn{2}{|c|}{ Hazard ratio for OS $(95 \% \mathrm{Cl})$} & $P$-value \\
\hline Higher age & $1+$ & $2.01(1.54,2.62)$ & $<0.001$ \\
\hline Lower HB & Int- & $1.56(1.14,2.14)$ & 0.005 \\
\hline Lower PLT & $t$ & $1.33(1.02,1.75)$ & 0.036 \\
\hline Higher blast $\%$ & $1+$ & $1.51(1.16,1.96)$ & 0.002 \\
\hline Higher $\mathrm{KT}$ risk & -1 & $1.65(1.15,2.35)$ & 0.006 \\
\hline Multi-hit TP53 & $\mapsto$ & $1.53(1.04,2.25)$ & 0.031 \\
\hline$D D \times 41$ & $\longrightarrow$ । & $0.35(0.19,0.63)$ & $<0.001$ \\
\hline $\operatorname{Del}(18 p)$ & $\mapsto$ & $1.62(0.96,2.73)$ & 0.070 \\
\hline$-7 / \operatorname{del}(7 q)$ & 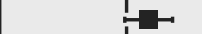 & $1.37(0.97,1.93)$ & 0.070 \\
\hline
\end{tabular}

Discovery cohort by IPSS-R:

VeryLow/Low/Intermediate

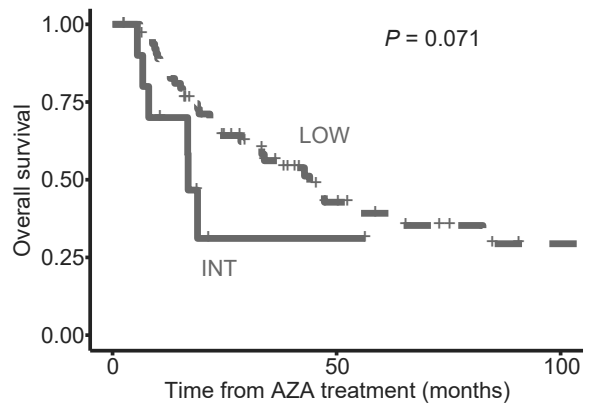

INT 11

LOW 65

Validation cohort according to new risk classes

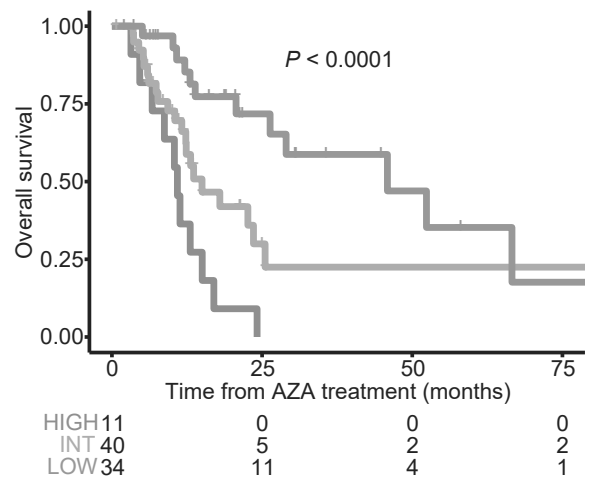


Extended Data Figure 5 | Factors associated with overall survival (OS).

a, Bar plots showing the generalized variance inflation factor (gVIF) of the covariates indicated on x-axis that are included in the multivariate Cox proportional hazards model for OS. Left and right panels show gVIF values before and after selection of parameter sets to circumvent multiple collinearity (Methods). b. A forest plot showing the result of Cox proportional hazards regression for overall survival (OS) on the discovery cohort with complete data for OS analysis $(n=341)$. Explicative variables are age, hemoglobin level, platelet level, marrow blast ratio (blast\%), karyotype (KT) risks, multi-hit TP53 mutation, DDX41 mutation, del(18p), and $-7 / \mathrm{del}(7 \mathrm{q})$. Age, hemoglobin, platelet count, blast\% were dichotomized with thresholds of 72-year-old, $80 \mathrm{~g} / \mathrm{L}, 50 \times 10^{9} / \mathrm{L}, 10 \%$, respectively. KT risks were classified into higher (poor and very poor) and lower (intermediate, good and very good) groups; the x-axis is log10 scaled. c, Kaplan-Meier estimates of OS per IPSS-R risks on the 320 cases in the discovery cohort with complete data for the analysis. The numbers of the cases at risk at each time are indicated in the tables below. $P$ value is derived from a two-sided log-rank test. d, Concordance index (C-index) of IPSS-R alone model and the novel scoring system for the discovery (left) and the validation (right) cohorts, respectively. e, Kaplan-Meier estimates of OS per risk groups of the novel scoring model for IPSS-R very low/low/intermediate (left panel), high (middle panel), and very high (right panel) risk groups, respectively. The numbers of the cases at risk at each time are indicated in the tables below. $P$ value is derived from a two-sided log-rank test. $\mathbf{f}$, Kaplan-Meier estimates of overall survival per risk classes of the proposed model for the validation cohort. Risk classes are determined according to the formula

presented in legends for Fig. 1d and Fig. 1e. The number of the cases at risk at each time is indicated in the tables below. $P$ values are derived from two-sided log-rank tests.

t-MN, treatment related myeloid neoplasm; AZA, azacitidine, CR, complete remission; mCR, marrow CR; PR, partial remission; SD, stable disease; HI, hematological improvement; PD, progressive disease; WT, wild type. 


\section{Extended Data Figure 6}

a

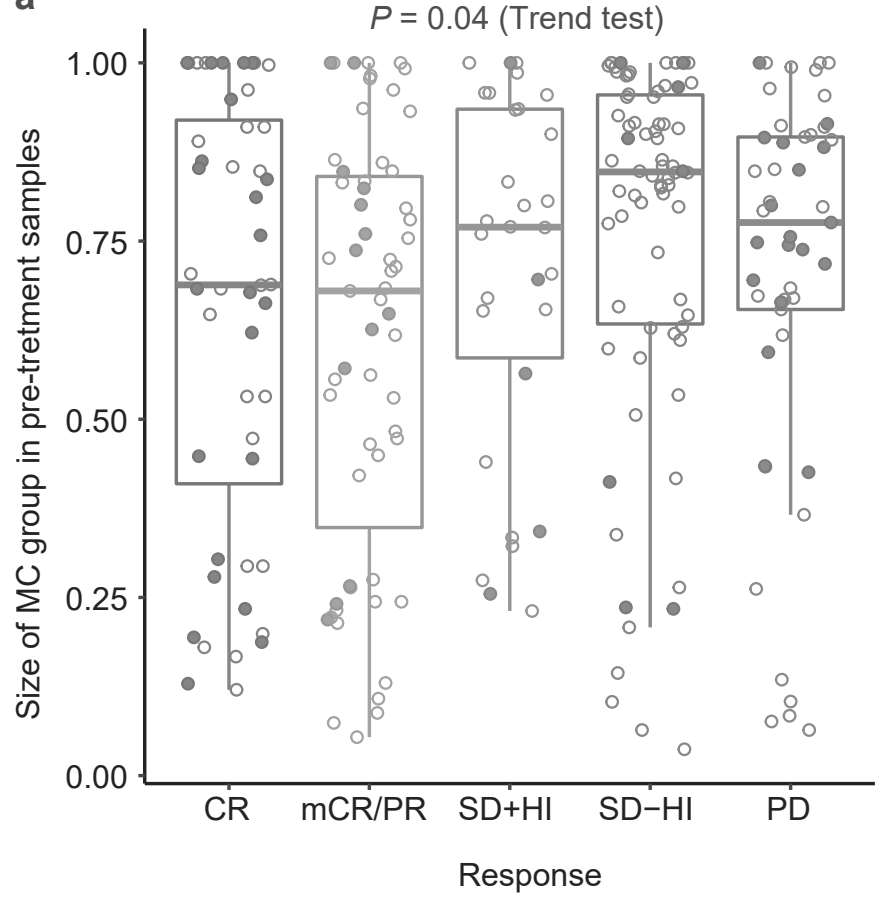

TP53-mutated

- No

- Yes b
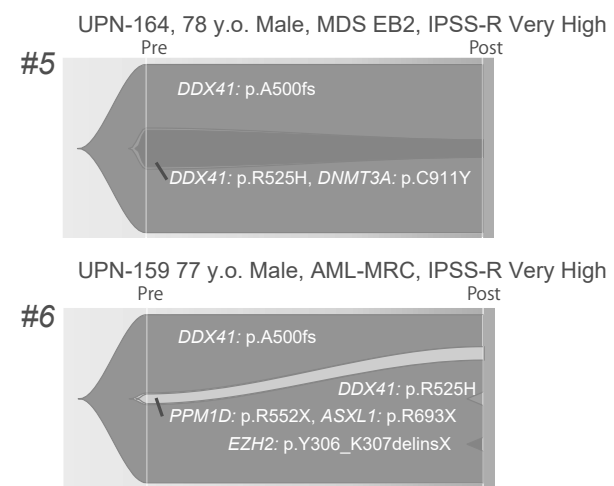

UPN-406 69 y.o. Male, MDS EB2, IPSS-R Very High

\#7
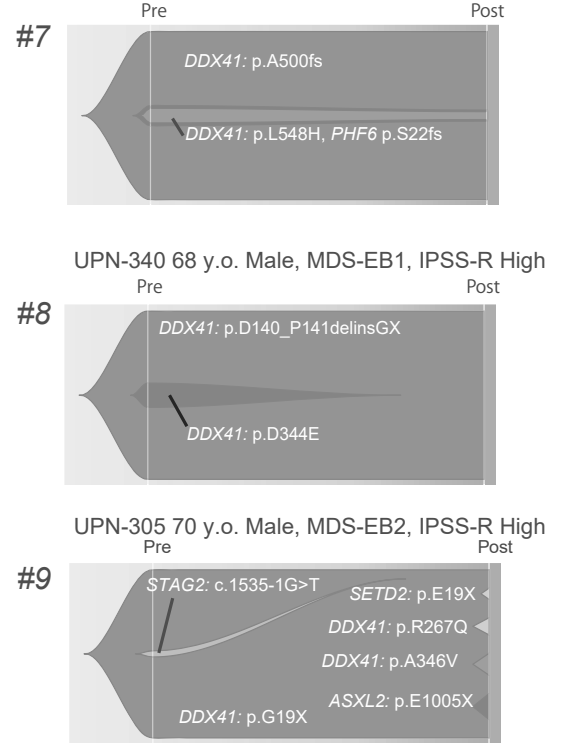

UPN-281 75 y.o. Male, MDS-RCMD, IPSS-R Very High

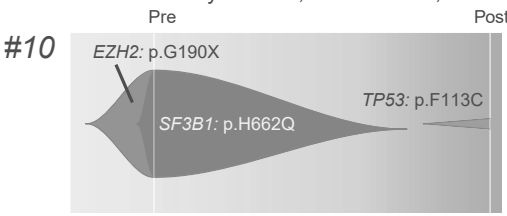

Extended Data Figure 6 | Major clone size in pre-treatment samples and clonal structure in stable disease (SD) cases with small post-treatment major clone (MC) size.

a, Box plots showing the MC group sizes of pre-treatment samples in the paired cohort having the response indicated on $\mathrm{x}$-axis. $P=0.04$, Jonckheere-Terpstra trend tests. $\mathbf{b}$, Fish plots showing estimated transition of the clone structure during azacitidine for the six cases with SD that had post-treatment MC group sizes $<0.10$. Sample IDs correspond to those indicated in Fig. 2c.

$\mathrm{CR}$, complete remission; mCR, marrow CR; PR, partial remission; HI, hematological improvement; PD, progressive disease. 


\section{Extended Data Figure 7}

a

\section{Whole genome sequencing of post-treatment samples and normal controls}

\section{Curation with the following criteria}

Depth $>30$

Not common SNPs $(1000 \mathrm{~g} 2014$ oct_all $<0.001)$

Fisher $P$ value $<10^{-1}$

EBCall $P$ value $<10^{-2}$

Number of supporting reads in tumor $>3$

Number of supporting reads in control $<5$

Somatic mutations list

b

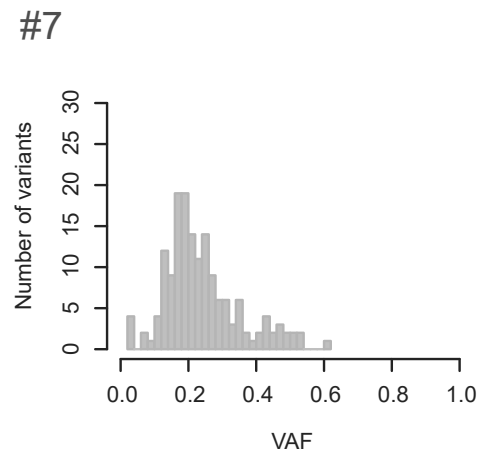

\#11

\#5
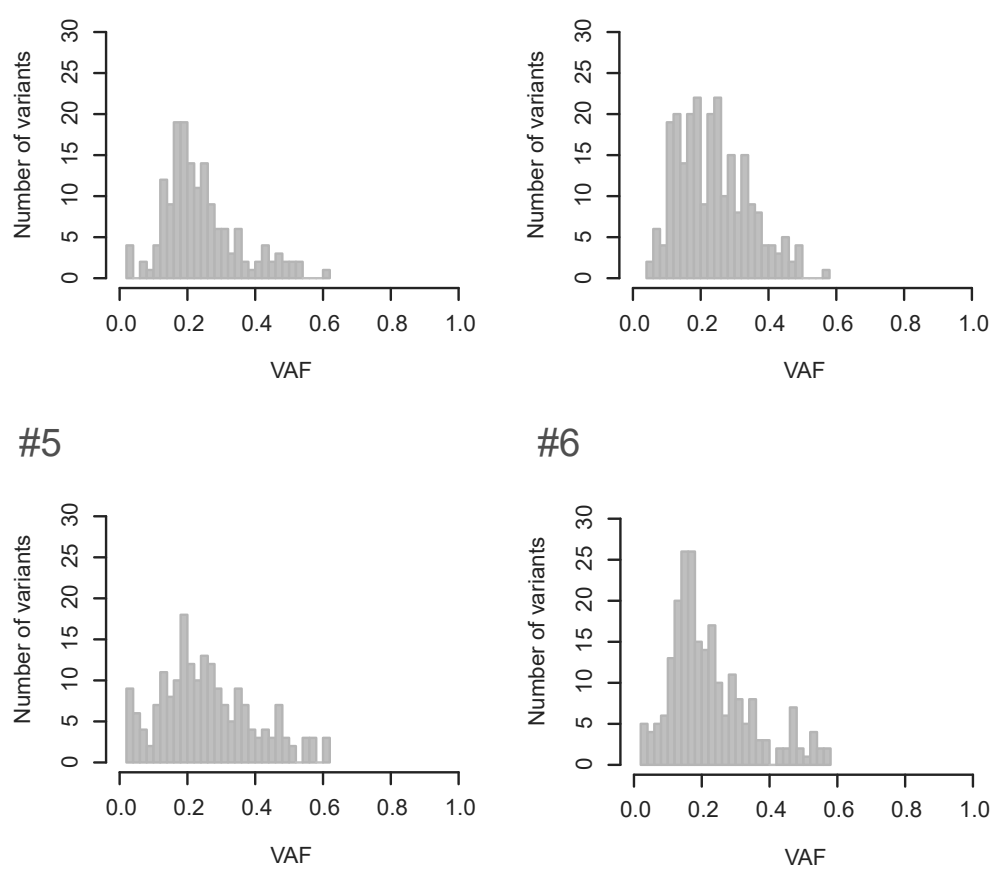

\#6

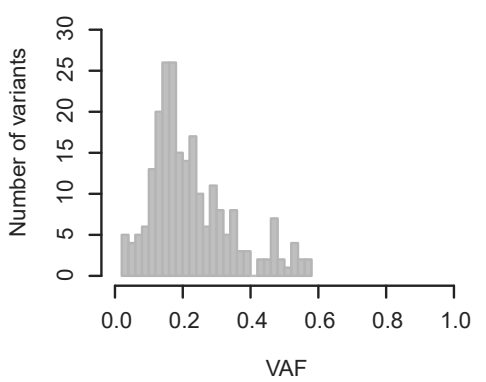

C

\begin{tabular}{|c|c|c|c|c|c|c|c|}
\hline Case & Age & Sex & Disease & IPSS-R & Response & $\begin{array}{l}\text { MC group size } \\
\text { (post treatment) }\end{array}$ & Post-treatment clones detected by panel sequencing (clone size) \\
\hline \#7 & 69 & Male & MDS EB2 & Very High & SD-HI & 0.057 & $D D X 41, \mathrm{pL} 548 \mathrm{H}(0.066)$, PHF6, p.S22fs $(0.047)$ \\
\hline \#11 & 72 & Female & MDS EB1 & Intermediate & $\mathrm{PD}$ & 0.094 & $D D X 41, \mathrm{pR} 525 \mathrm{H}(0.094)$ \\
\hline \#5 & 78 & Male & MDS EB2 & Very High & $\mathrm{SD}+\mathrm{HI}$ & 0.086 & $D D X 41, \mathrm{pR} 525 \mathrm{H}(0.068)$, DNMT3A, p.C911Y (0.104) \\
\hline \#6 & 77 & Male & AML-MRC & - & SD-HI & 0.082 & $\begin{array}{l}\text { PPM1D, p.R552X (0.082), ASXL1, p.R693X (0.082), DDX41, } \\
\text { p.R525H (0.080), EZH2, p.Y306_K307delinsX (0.080) }\end{array}$ \\
\hline
\end{tabular}

Extended Data Figure 7 | Whole genome sequencing to search for potential drivers.

We conducted whole genome sequencing for the 4 cases that had no overall response after azacitidine treatment although MC group sizes were $<0.20$. All these cases had germline $D D X 41$ mutations. a, Filtering condition of somatic mutations of whole genome sequencing to curate somatic mutations. b. Variant allele frequency (VAF) histogram of somatic mutations of the 4 cases. Only single nucleotide variants were included from the somatic mutation list because indels show deviated VAF values from true ratio of altered alleles. c, A table showing the clinical and post-treatment clonal composition of the 4 cases. All these cases show small major clone (MC) group sizes in post-treatment samples. 1000g2014oct_all, 1000 Genomes Project (2014 Oct) annotations; EBCall, an empirical Bayesian framework for somatic mutation call. See Nucleic Acids Res 41:e89, 2013; SD, stable disease; HI, hematological improvement; PD, progressive disease. 


\section{Extended Data Figure 8}

a

OS of transplanted cases

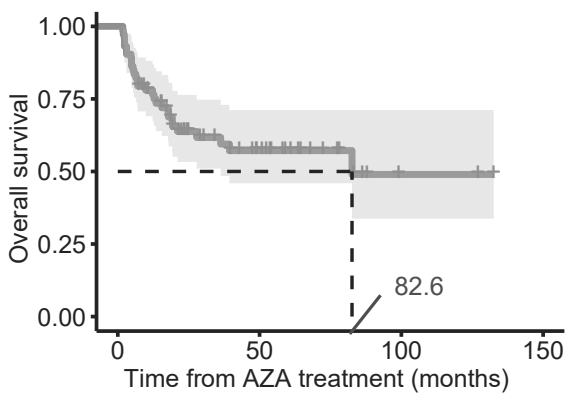

Number at risk

73

20

C

OS of transplanted cases with multihit TP53 mutations

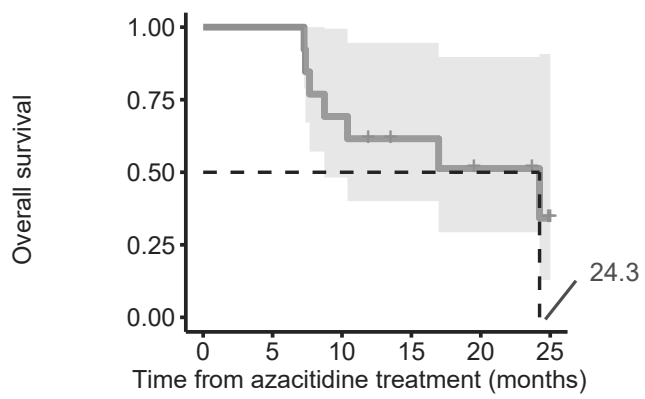

Number at risk

$\begin{array}{llllll}13 & 13 & 9 & 6 & 4 & 1\end{array}$ b

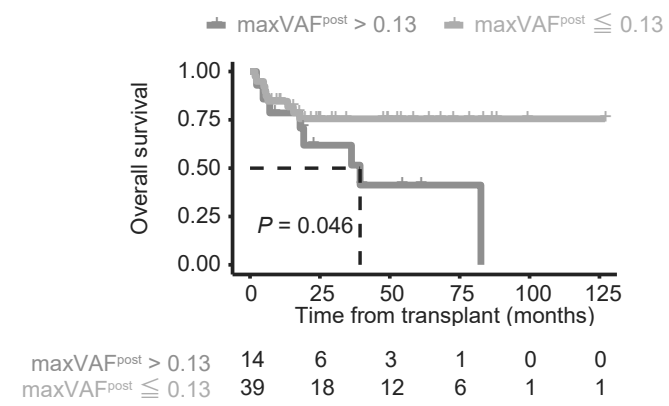

d OS of transplanted cases with multihit TP53 mutations

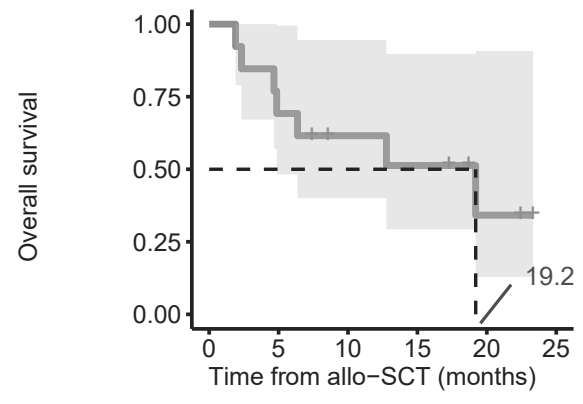

Number at risk

$\begin{array}{llllll}13 & 9 & 6 & 5 & 2 & 0\end{array}$

Extended Data Figure 8 | Outcomes of transplanted cases.

a, Kaplan-Meier estimates of OS on the 73 cases who received allogeneic stem cell transplantation (allo-SCT). b, Kaplan-Meier estimates of OS per maxVAFpost values $(>0.13$ or $<=0.13)$ on the 53 cases who received allogeneic stem cell transplantation (allo-SCT) after azacitidine treatment. The numbers of the cases at risk at each time are indicated in the table below. $P$ value is derived from a two-sided log-rank test. c, d, Kaplan-Meier estimates of OS on the 13 cases with TP53 mutations who received allo-SCT. Time is calculated after azacitidine administration (c) or allo-SCT (d). The numbers of the cases at risk at each time are indicated in the table below. 


\section{Extended Data Table 1}

\begin{tabular}{|c|c|c|c|c|c|c|}
\hline \multirow[b]{3}{*}{ Sample } & \multirow[b]{3}{*}{ TP53 } & \multirow{3}{*}{$\begin{array}{l}\text { PPM1D } \\
\text { Variant }\end{array}$} & \multicolumn{4}{|c|}{ Pre-treatment } \\
\hline & & & \multirow[b]{2}{*}{$\begin{array}{c}\text { Detected by } \\
\text { pipeline }\end{array}$} & \multicolumn{3}{|c|}{ IGV } \\
\hline & & & & VAF & $\begin{array}{c}\text { Number of } \\
\text { supporting } \\
\text { reads }\end{array}$ & $\begin{array}{c}\text { Number } \\
\text { of total } \\
\text { reads }\end{array}$ \\
\hline \#PPM1D-1 & Multihit & p.S432fs & Yes & 0.046 & 25 & 548 \\
\hline \#PPM1D-2 & Multihit & p.1436fs & No & 0.005 & 7 & 1367 \\
\hline \#PPM1D-3 & Multihit & p.P471fs & No & 0.001 & 2 & 1429 \\
\hline \#PPM1D-4 & Multihit & p.N477fs & No & 0.011 & 10 & 938 \\
\hline \#PPM1D-5 & $\mathrm{Wt}$ & p.C478X & No & 0.000 & 0 & 883 \\
\hline \#PPM1D-6 & 1-hit & p.D488fs & No & 0.000 & 0 & 2138 \\
\hline \#PPM1D-7 & Wt & p.M508fs & No & 0.005 & 10 & 2107 \\
\hline \#PPM1D-8 & Wt & p.R552X & No & 0.003 & 2 & 589 \\
\hline \#PPM1D-9 & Multihit & p.R572X & No & 0.001 & 1 & 924 \\
\hline
\end{tabular}

Extended Data Table 1 | Strenuous search for PPM1D-mutated clones by integrative genomics viewer (IGV) in pre-treatment samples that expanded their sizes in post-treatment samples.

A table showing the result of strenuous search for the supporting reads in pre-treatment samples by IGV. Case IDs correspond to those presented in Fig. 4e.

VAF, variant allele frequency; WT, wild type. 


\section{Supplementary Appendix}

Supplementary Data

Supplementary Figures

Supplementary Figure 1| Summary of the study cohorts subjected to each analysis.

Supplementary Figure 2 | Quality control of targeted sequencing.

Supplementary Figure 3 | Mutation Profile of discovery and validation cohorts.

Supplementary Figure 4 | Paired box plots showing clone sizes in pre- and post-treatment samples.

Supplementary Figure 5 | Fish plots of CR cases harboring mutations in RUNX1.

Supplementary Tables

Supplementary Table 1 | Panel of genes applied in this study.

Supplementary Table 2 | List of pathogenic variants in the pre-treatment samples.

Supplementary Table 3 | List of pathogenic variants in the post-treatment samples.

Supplementary Table 4 | List of CNVs in the pre-treatment samples.

Supplementary Table 5 | List of CNVs in the post-treatment samples.

Supplementary Table 6 | List of DDX41 mutations with VAF values and expected genetic mode.

Supplementary Table 7 | List of somatic mutations in 4 WGS for germline DDX41-mutated cases with poor response. 
Supplementary data

Details of study cohort

JALSG-MDS-201

JALSG-MDS-212 study is a multi-center prospective, randomized phase 3 trial conducted by Japan Adult Leukemia Study Group (JALSG). The major inclusion criteria is myelodysplastic syndromes (MDS) refractory anemia with excess of blasts (RAEB) or RAEB in transformation (RAEB-T) in FAB classification ${ }^{2}$. The patients were excluded who were assumed to be candidates for allogeneic stem cell transplantation (allo-SCT), who is younger than 16 years old, who has dysfunction in major organs (liver, kidney, heart, and lung), and who had received any of the following therapies: azacitidine, anti-cancer drugs, allo-SCT, lenalidomide. The registered subjects were randomly assigned to either of 5-day and 7-day schedule of azacitidine at $75 \mathrm{mg} / \mathrm{m}^{2}$ daily dose. The primary endpoint is to examine the non-inferiority of 5-day cohort to 7-day cohort in two-year overall survival (OS). This study is registered at UMIN-CTR (UMIN000009633). We excluded the subjects from this study when the patient dropped out before start of azacitidine, both response and survival data are missing, genetic data were not available for pre-treatment samples. Although marrow blast ratio increased $>30 \%(34 \%$ and $37 \%)$ in 2 subjects between enrollment to JALSG-MDS212 study and start of azacitidine, they were included in this study following the intention-to-treat policy of JALSG-MDS212 study. Both of the 2 cases had germline DDX41 mutations.

Karolinska University retrospective cohort.

Retrospectively collected consecutive patients with MDS, MDS/MPN, and oligoblastic secondary AML (marrow blast $<30 \%$ and transformed from MDS or MDS/MPN) who had received at least 1 cycle of azacitidine were included. Patients were excluded from this study who received azacitidine therapy as a consolidation therapy following intensive chemotherapies or as a palliative treatment. The patients were treated uniformly according to the European label.

The validation cohort.

For the validation cohort, we enrolled subjects from the hematological malignancy biobank at Kyoto University, collected from collaborative institutes in Japan. We enrolled the cases meeting the following criteria: 1) MDS, MDS/MPN, or AML-MRC < 30\% blast that have progressed from MDS or MDS/MPN. 2) received at least one 
course of azacitidine. 3) azacitidine was not administered as a consolidation therapy following intensive chemotherapies or as a palliative treatment. 


\section{Supplementary Fig. 1}

a

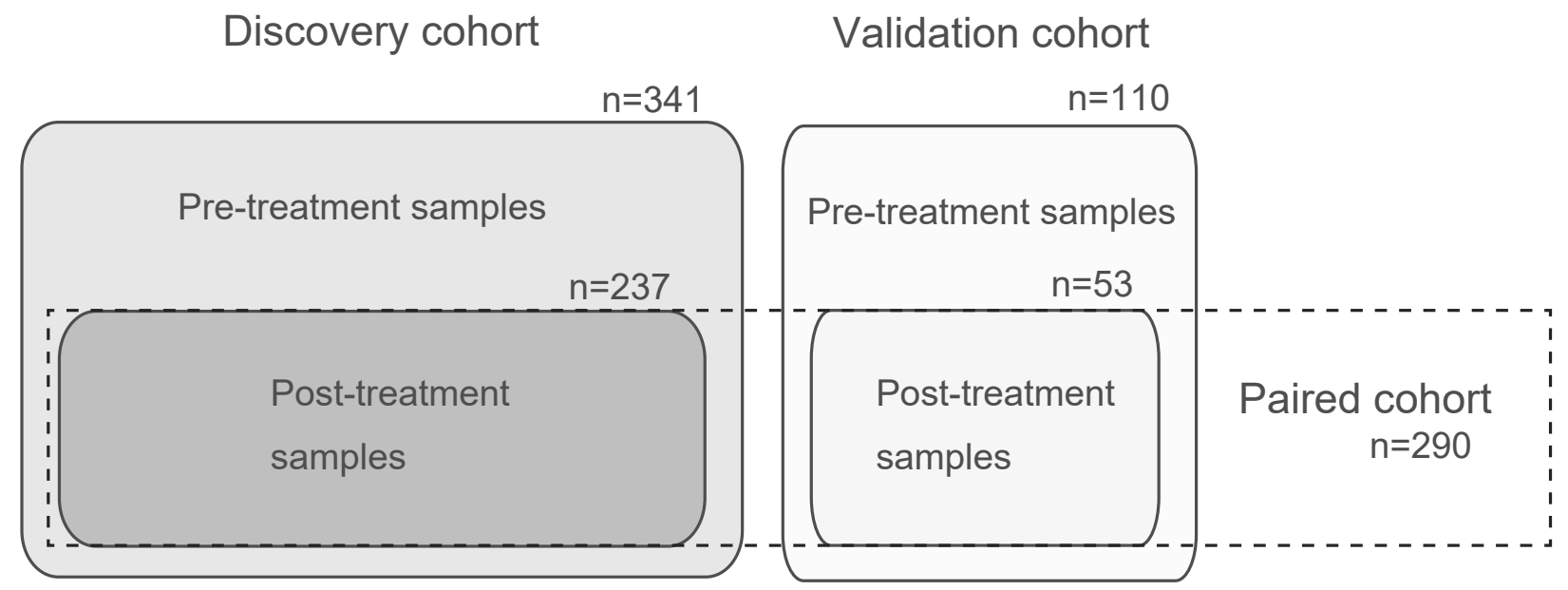

b

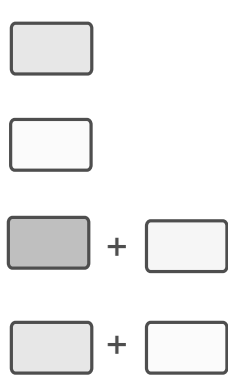

: Fig. 1, Extended Data Figs. 2-5 (except for Extended Data Fig. 2b, Extended Data Fig. 4b, Extended Data Fig. 5f)

: Extended Data Fig. 2b, Extended Data Fig. 4b, Extended Data Fig. 5d, $5 f$

: Fig. 2, Fig. 3, Fig. 4, Fig. 5a-d, Extended Data Fig. 6-7

: Fig. $5 f$ (Transplanted cases), Extended Data Figs. 8

Supplementary Fig. 1 | Summary of the study cohorts subjected to each analysis.

a, The discovery cohort was enrolled from JALSG-MDS-212 prospective study $(n=178)$ and from consecutively collected retospectivretospective cohort from Karolinska Institute $(n=163)$. As a validation cohort, additional 110 cases were retrospectively collected biobank of hematological malignancies at Kyoto University. Pre-treatment samples were collected and subjected for sequencing analysis. Among them, post-treatment samples were also subjected for sequencig analysis for 237 and 53 cases in the discovery and the validation cohort, respectively. b, Illustrative explanation showing the cohorts that were subjected to each analysis represented by the indicated data. 


\section{Supplementary Fig. 2}

a

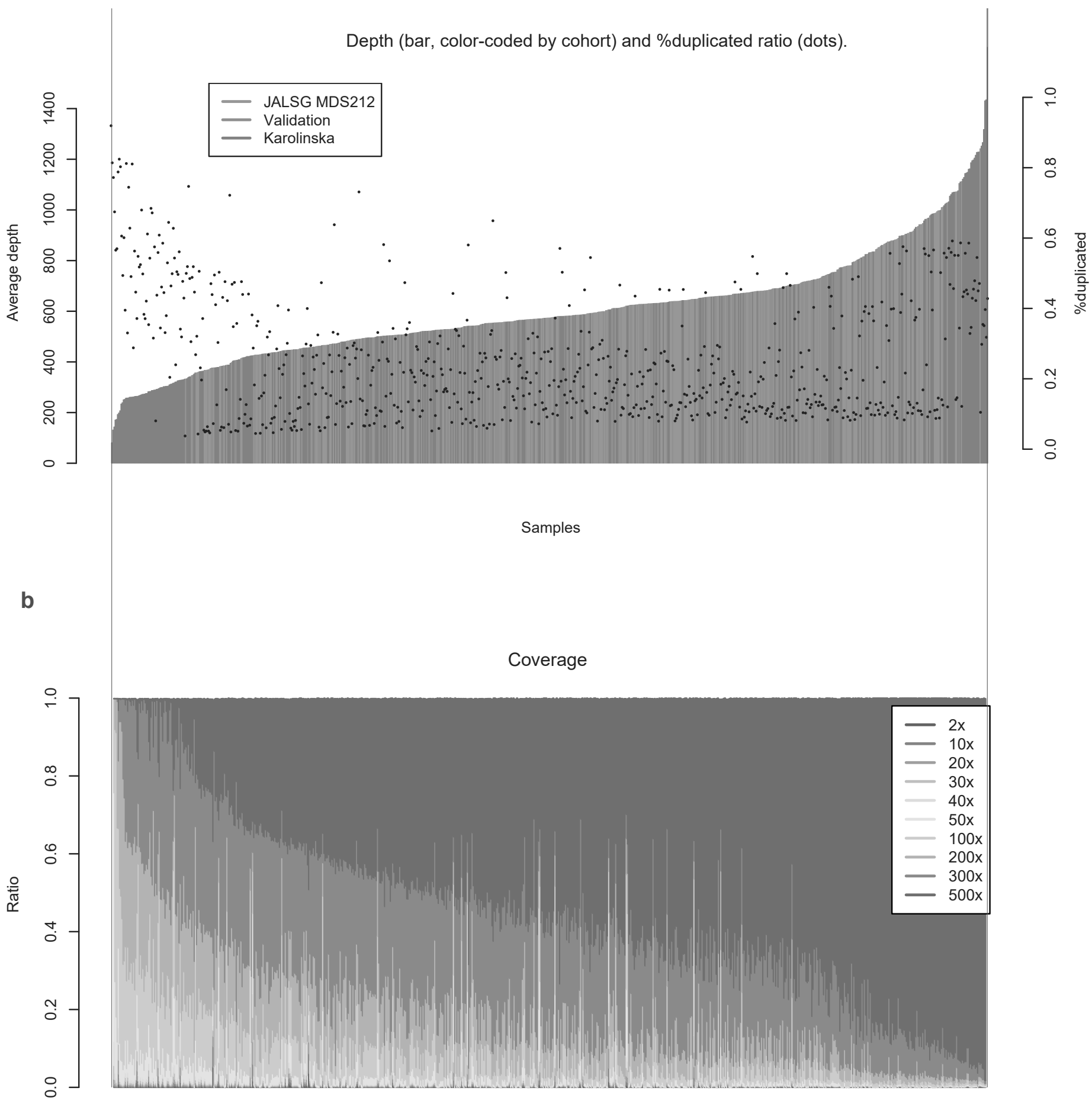

Samples

\section{Supplementary Fig. 2 | Quality of sequencing.}

a, Depth and \%duplicated ratio of the targeted sequencing. Samples are arranged in the ascending order of average depth and color-coded by the cohort. $\mathbf{b}$, Ratio of the targeted genomic region covered by the depths indicated in each color, where samples are arranged in the same order in the panel a. 


\section{Supplementary Fig. 3}

a Pre-treatment samples
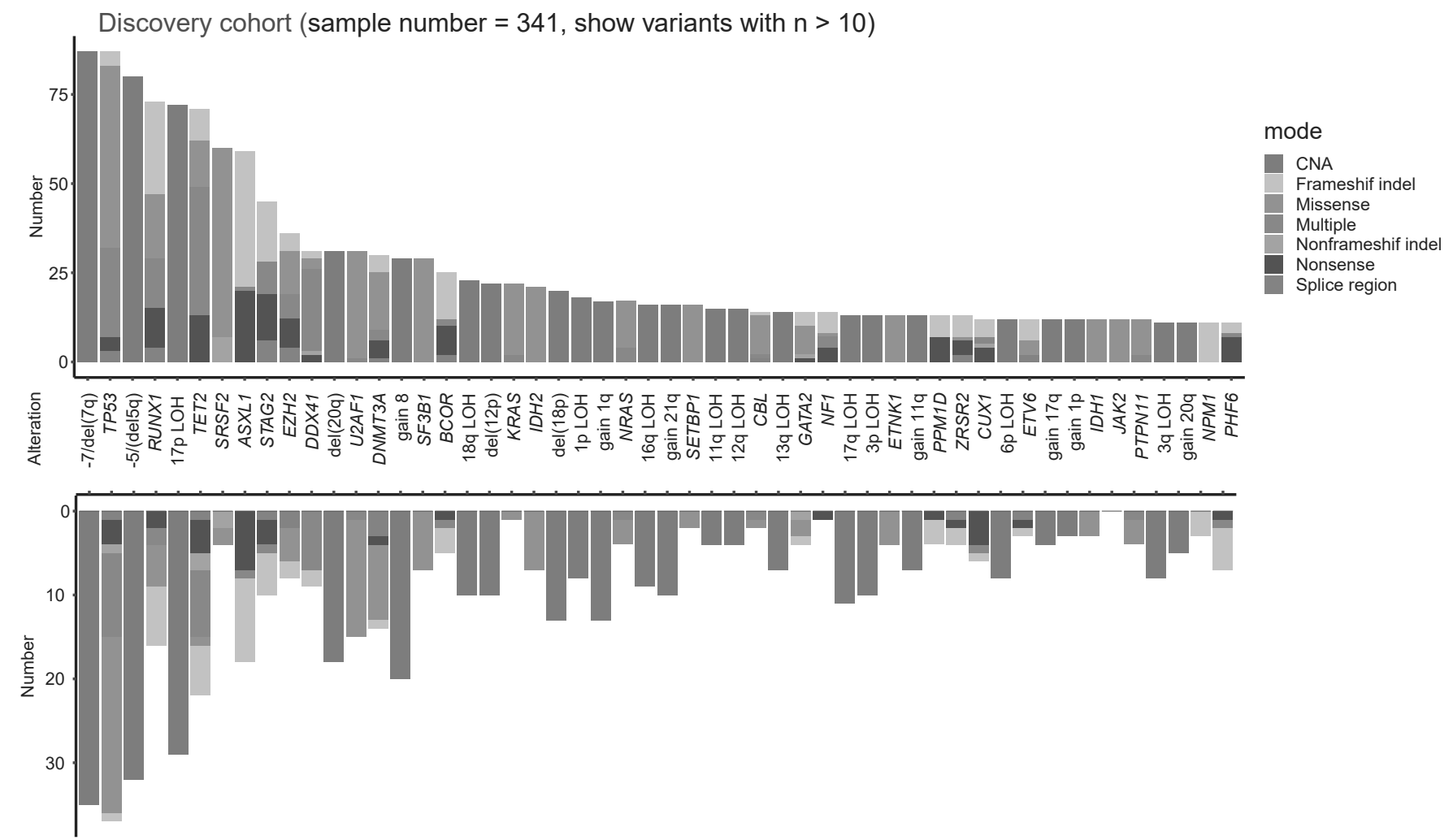

Validation cohort, (sample number=110)

b Post-treatment samples
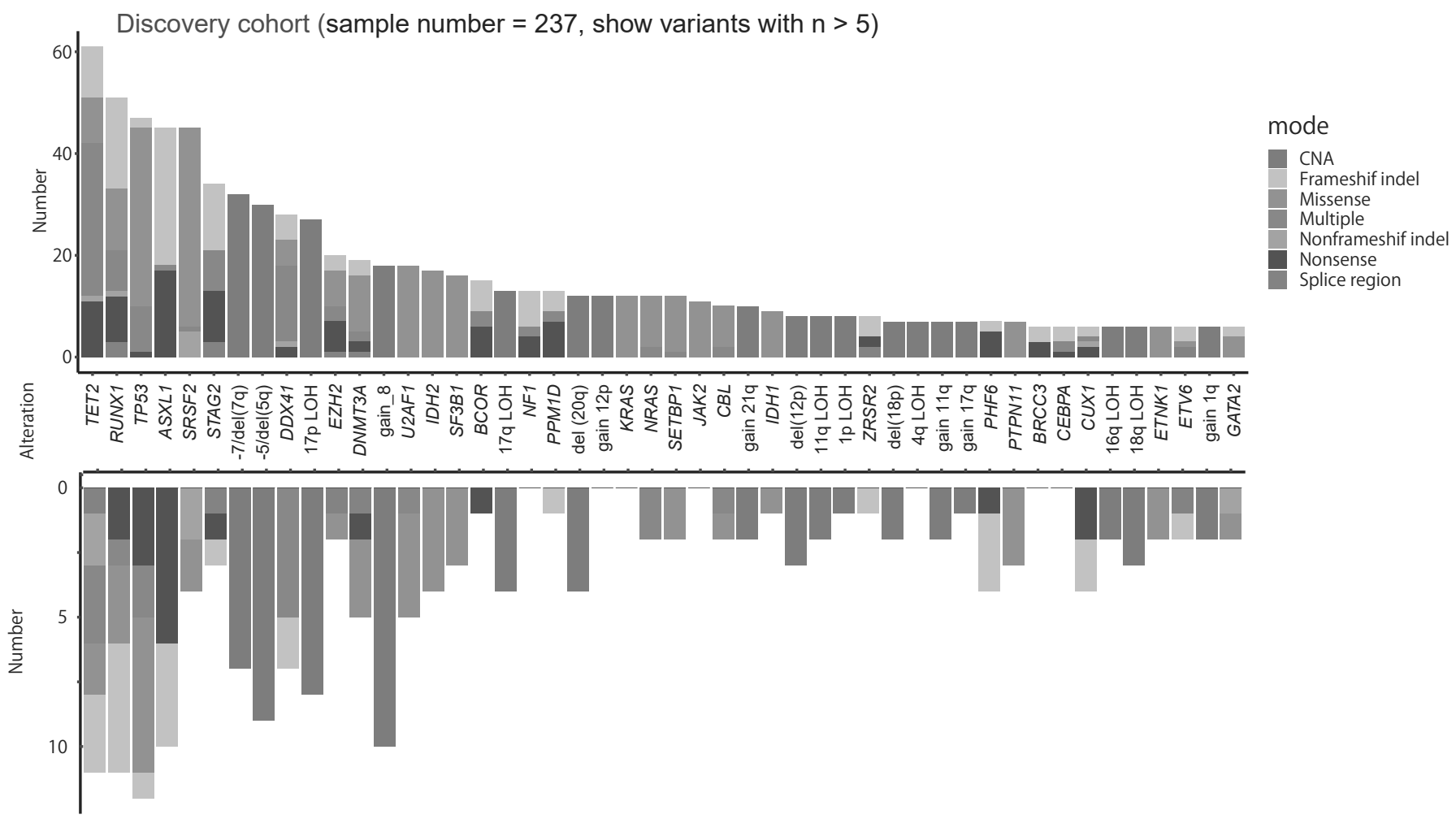

Validation cohort, (sample number=53)

\section{Supplementary Fig. 3 | Distribution of frequently observed genetic alterations.}

The distribution of genetic alterations detected in discovery cohort (upper panel) and validation cohort (lower panel) in pre-treatment samples (a), and post-treatment samples (b). Y-axes of the bar plots indicate the number of samples harbouring the corresponding variants. CNA, copy number alteration; $\mathrm{LOH}$, loss of heterozygosity. 
Supplementary Fig. 4

TET2

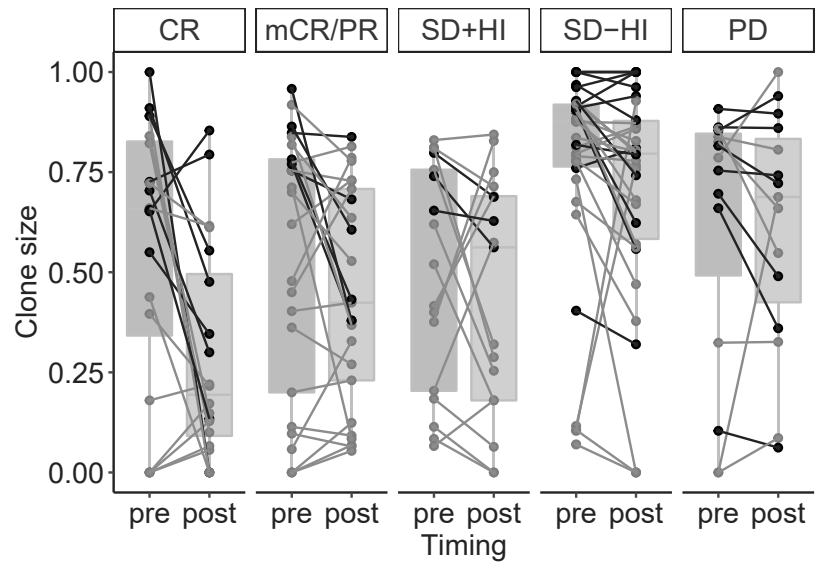

RUNX1

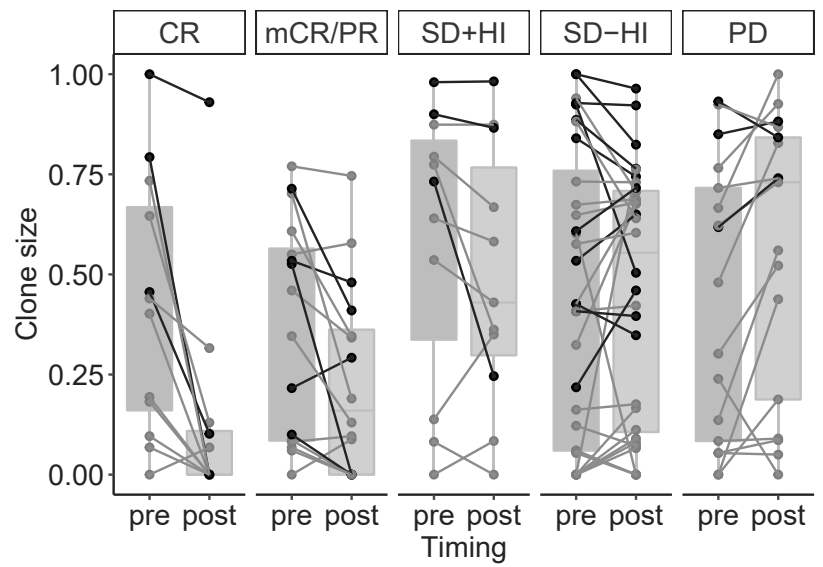

SRSF2

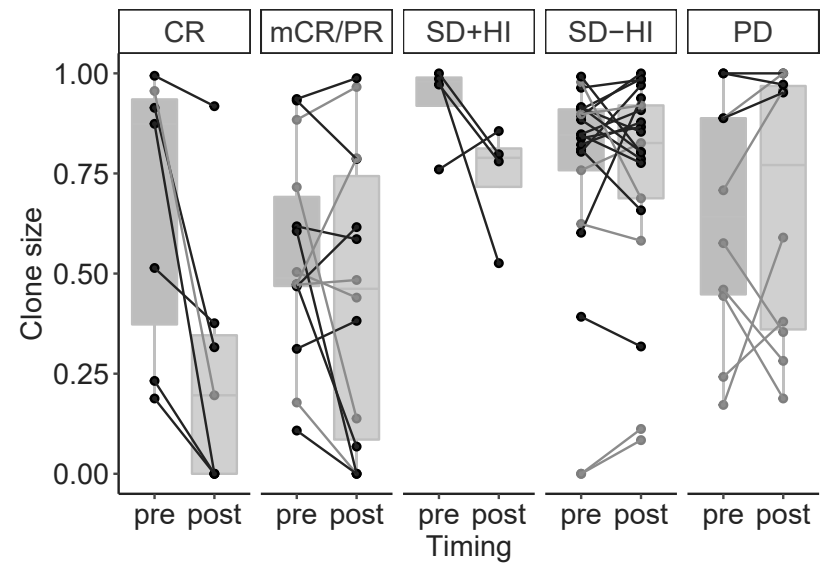

EZH2

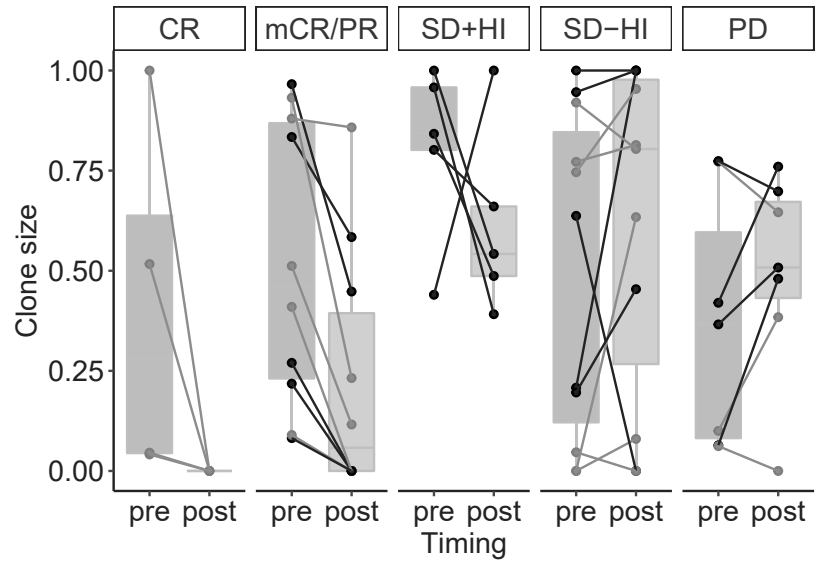

TP53

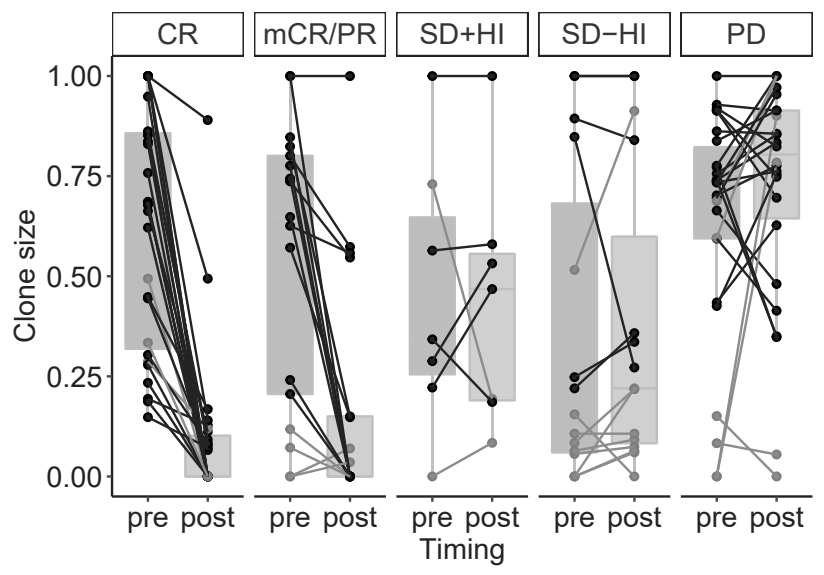

ASXL1

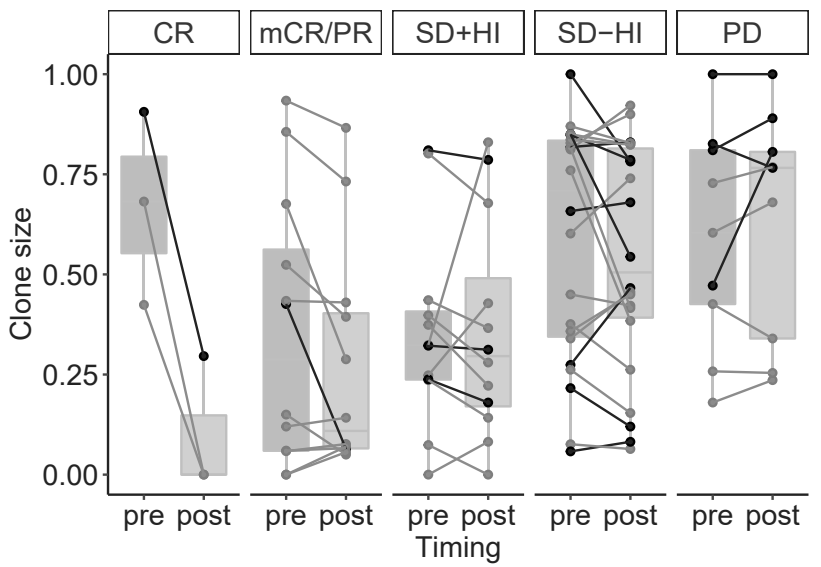

STAG2

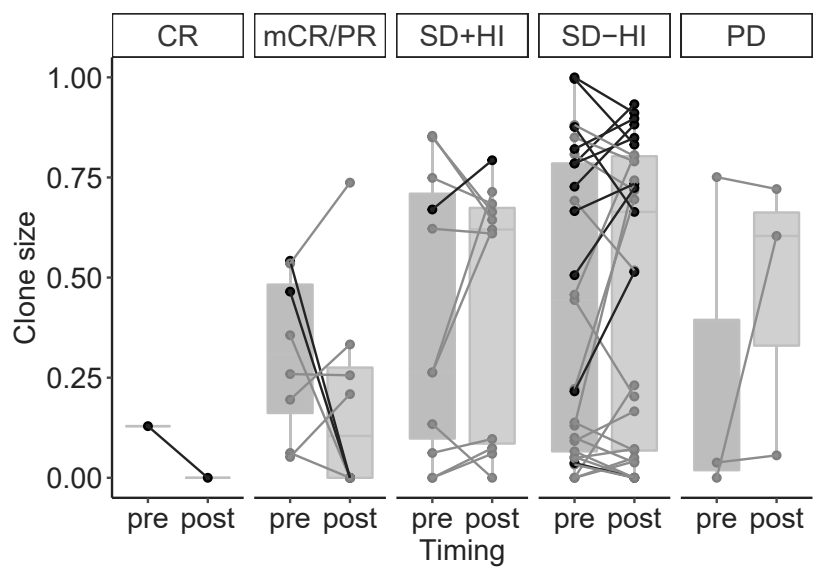

DNMT3A

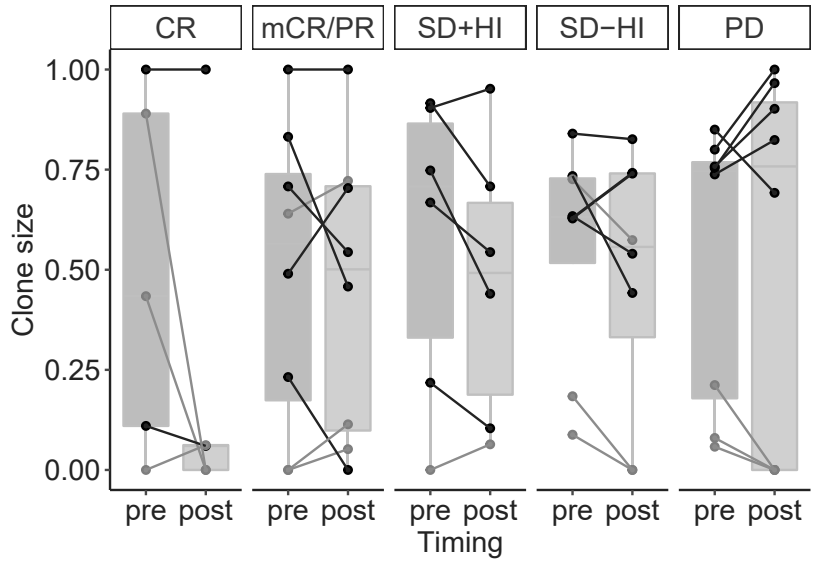


DDX41

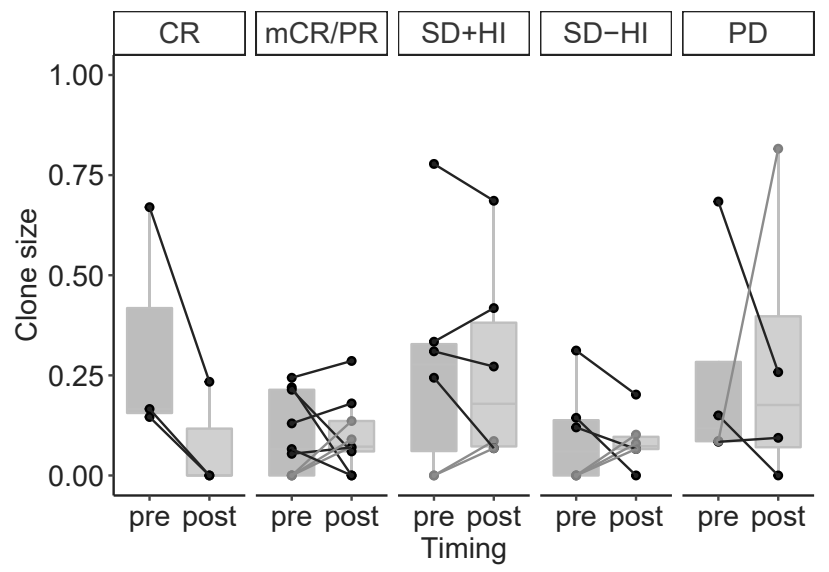

BCOR

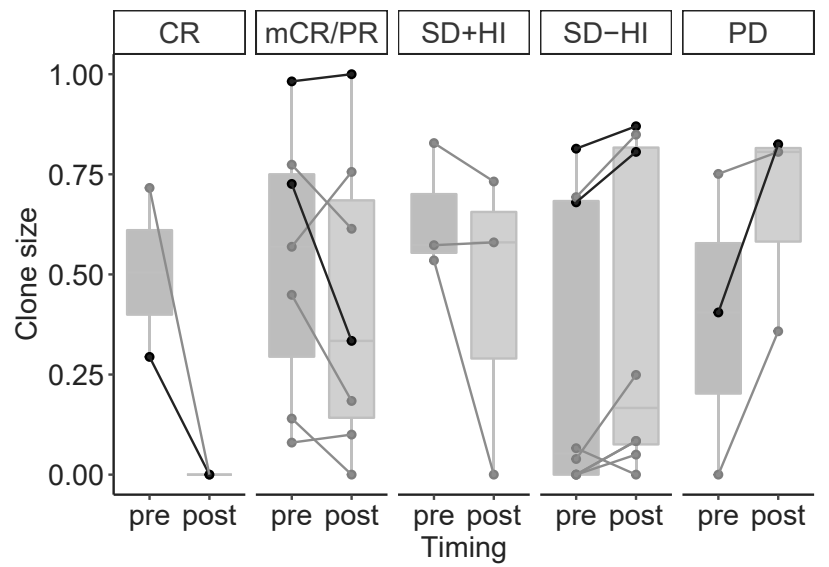

NRAS

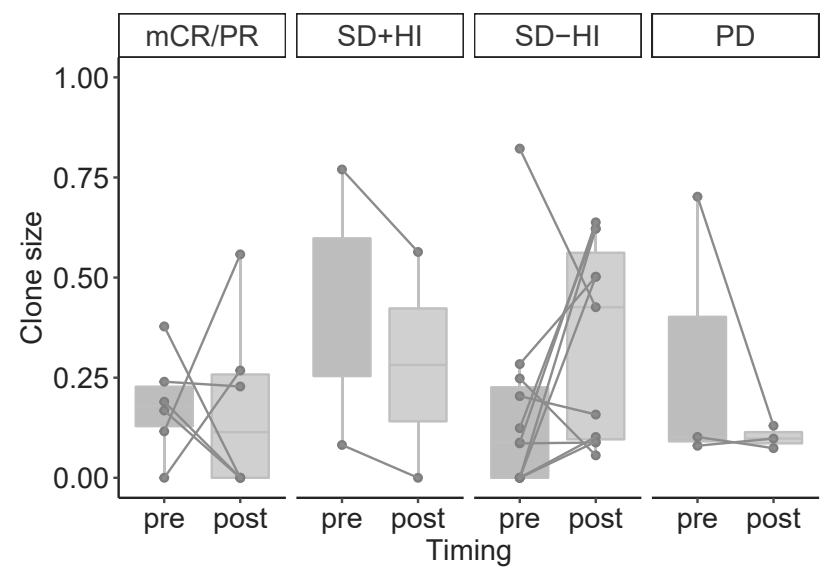

IDH2

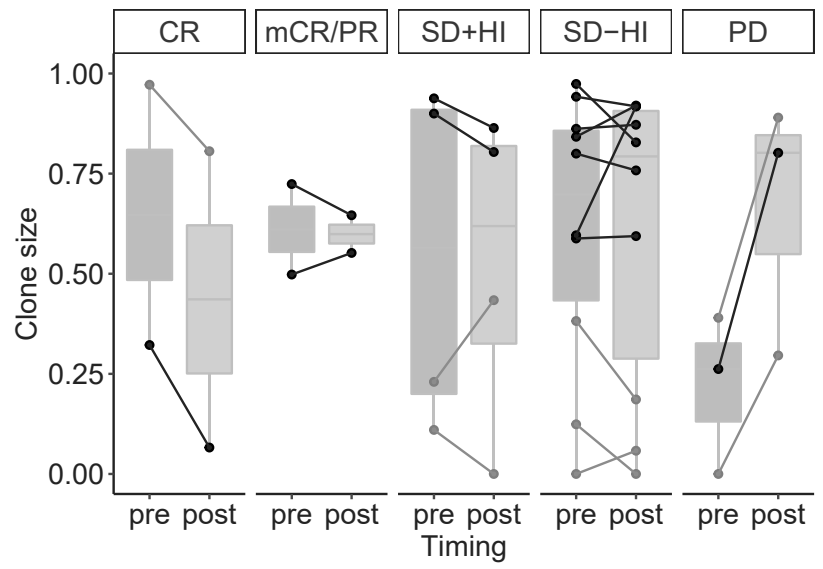

U2AF1

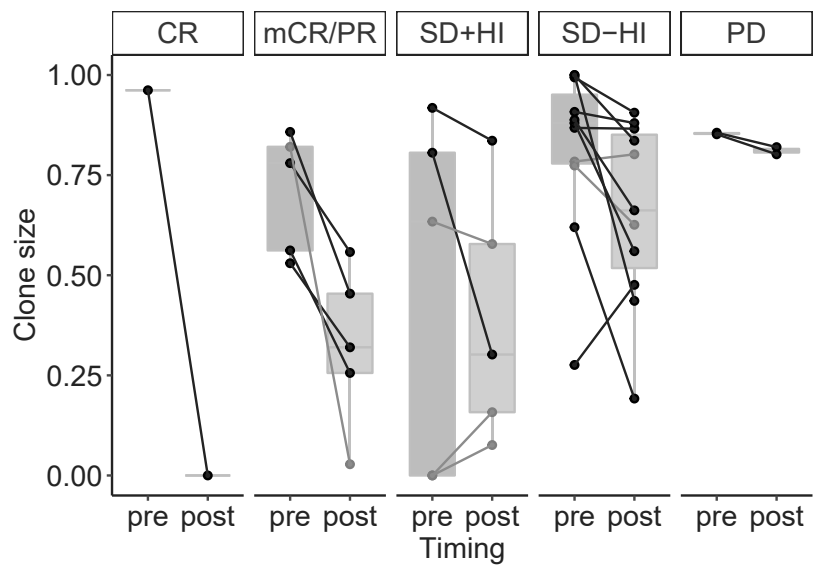

KRAS

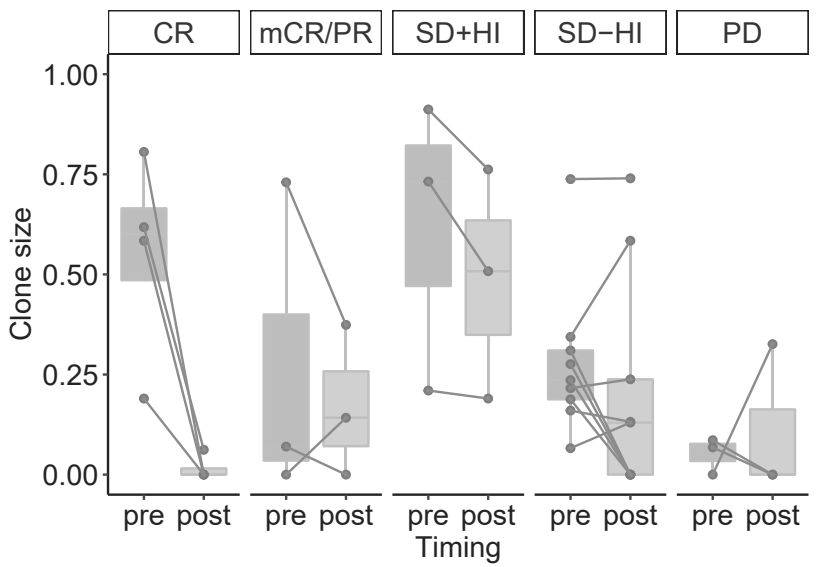

CBL

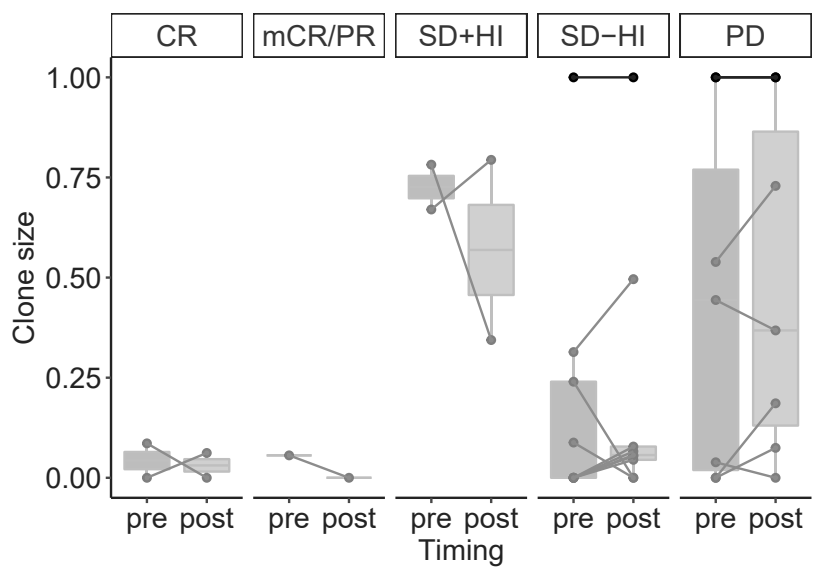

SF3B1

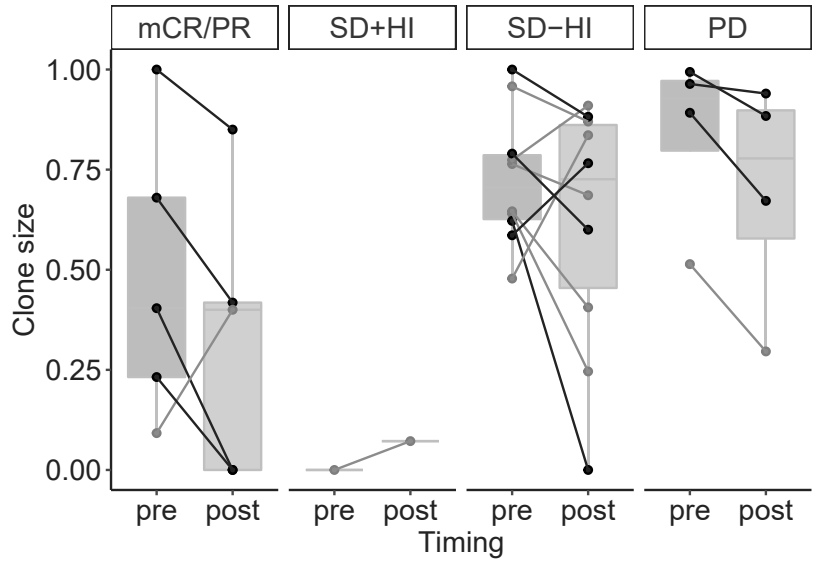



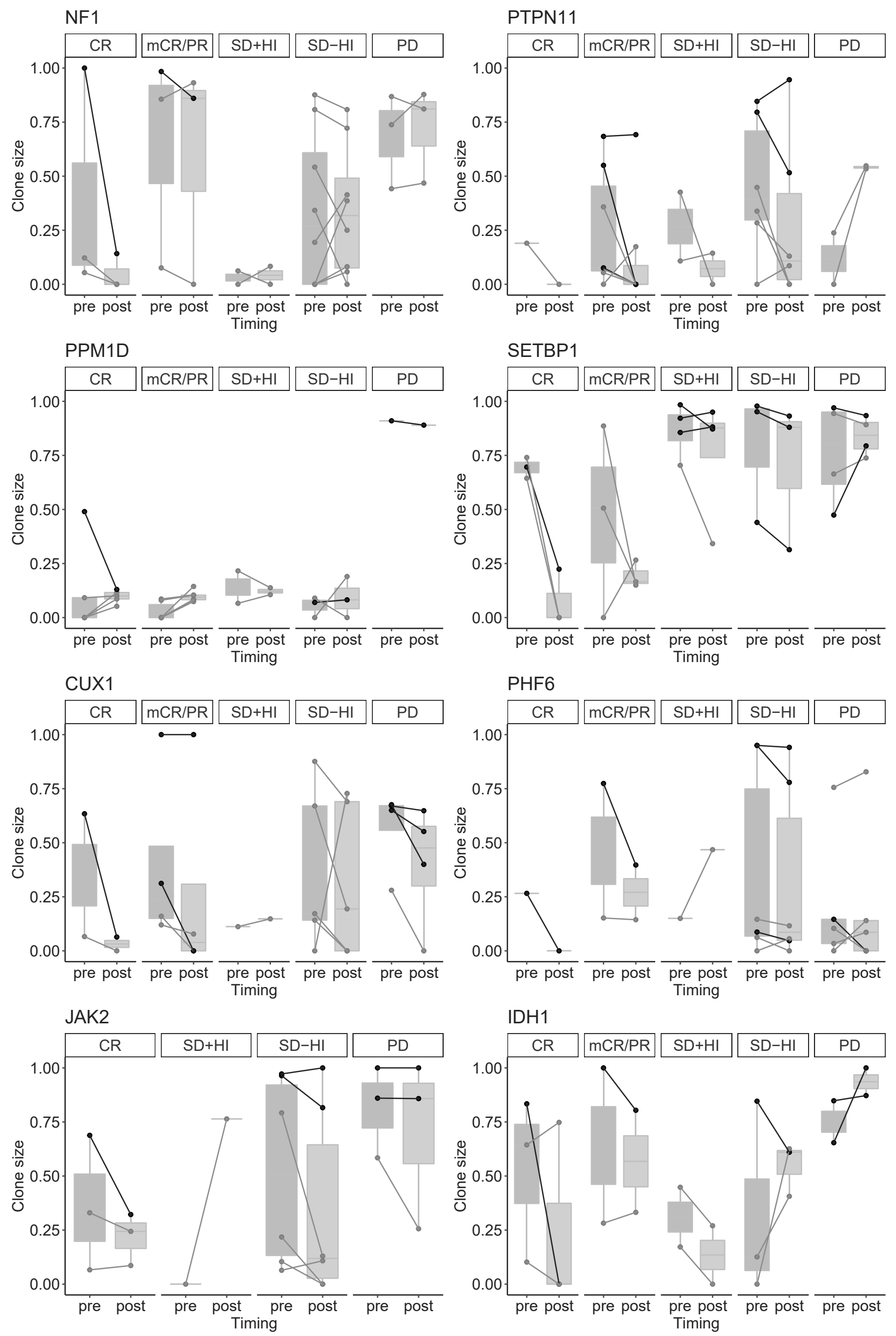
CEBPA

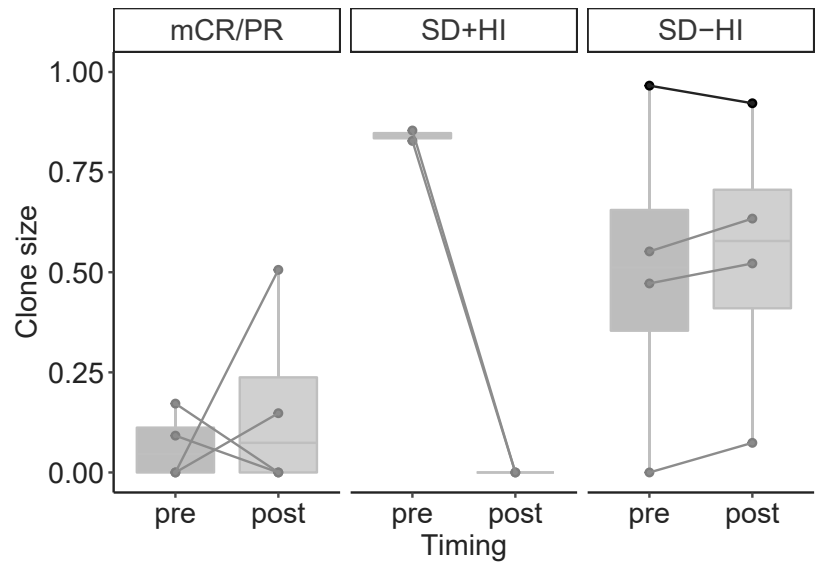

ETV6

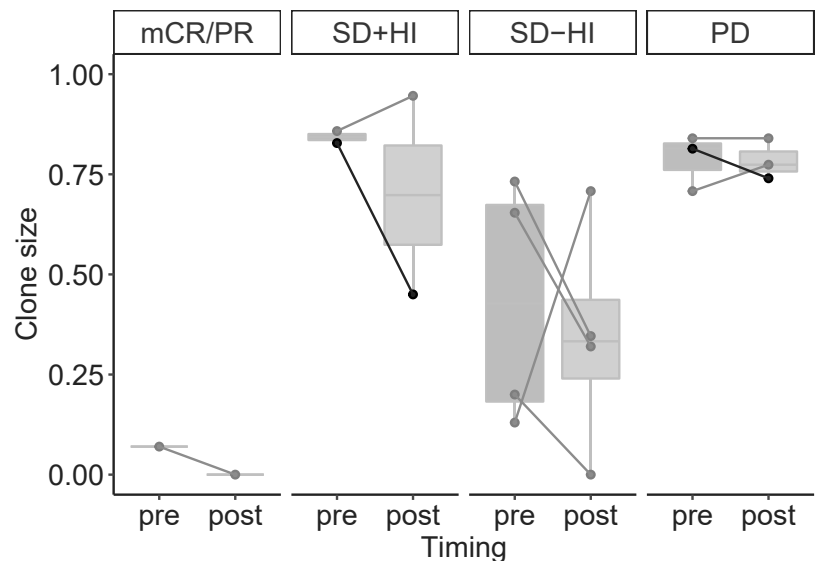

ZRSR2

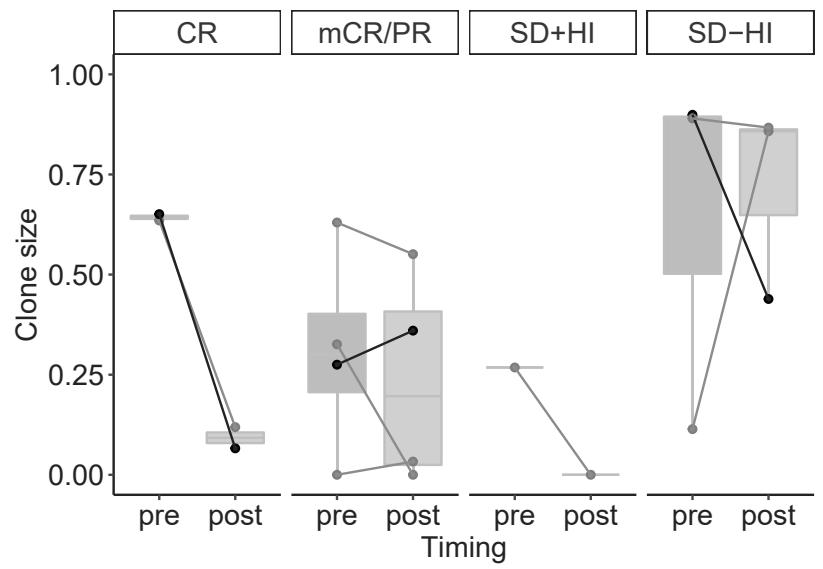

SMC3

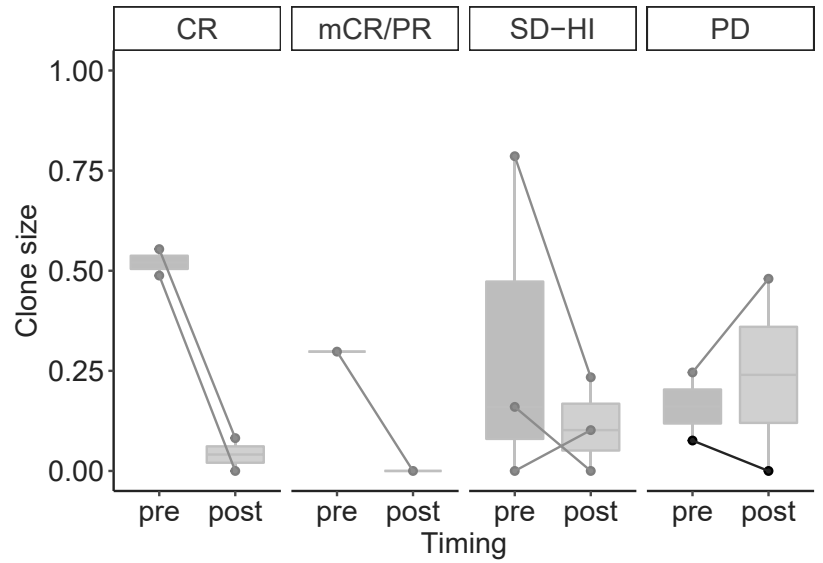

ETNK1

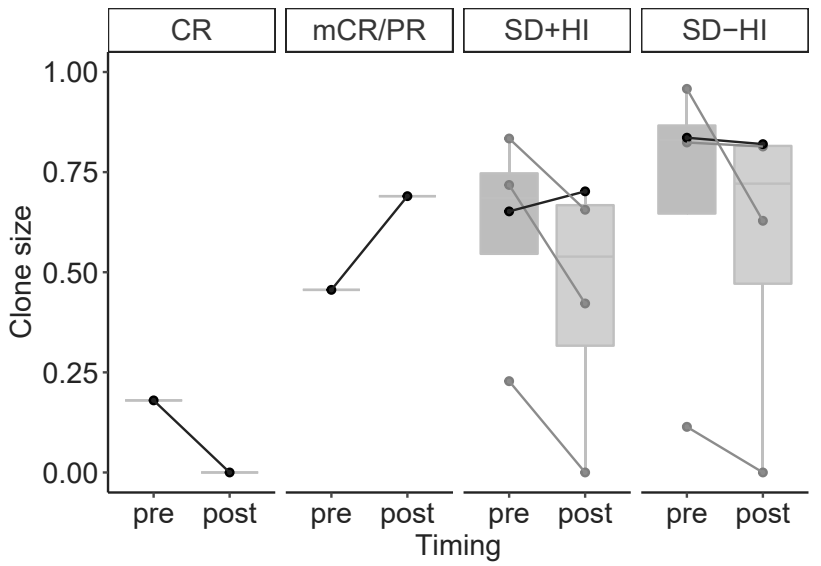

GATA2

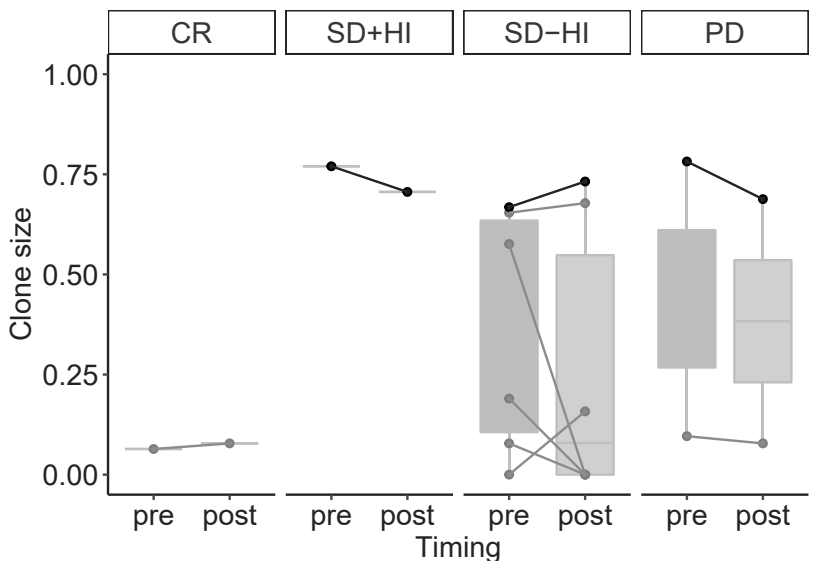

NPM1

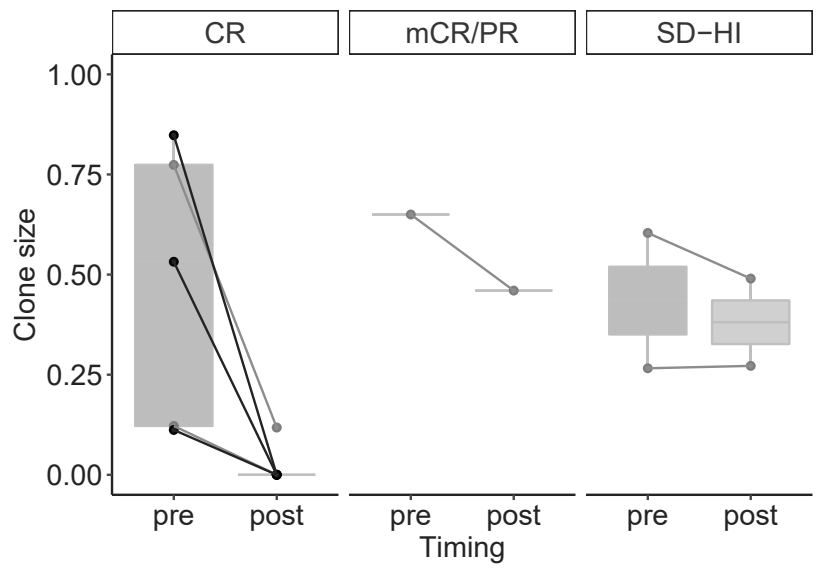

WT1

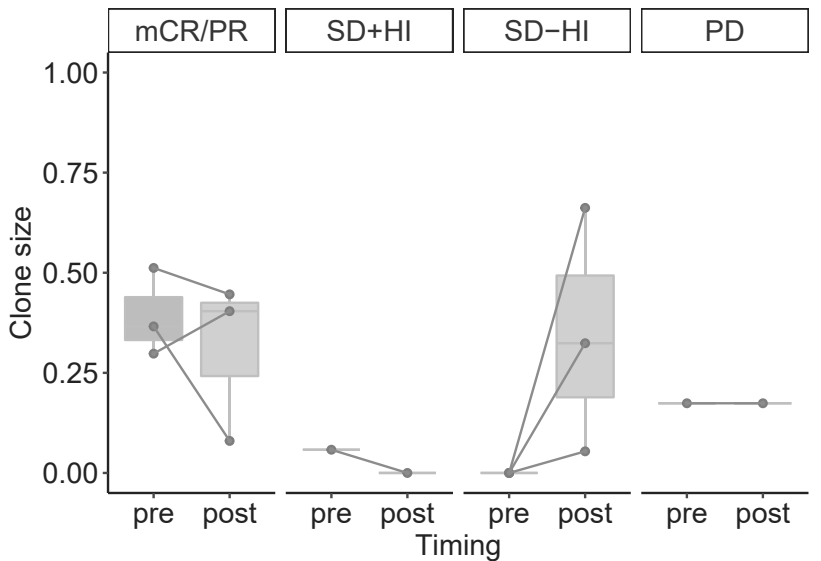


Supplementary Fig. 4 | Paired box plots showing clone sizes in pre- and post-treatment samples.

Paired box plots showing the change in clone sizes represented by indicated genes. The data are shown for each response and colors of line indicate whether the clone is classified as a major clone (MC, black lines) or not (red lines). 


\section{Supplementary Fig. 5}

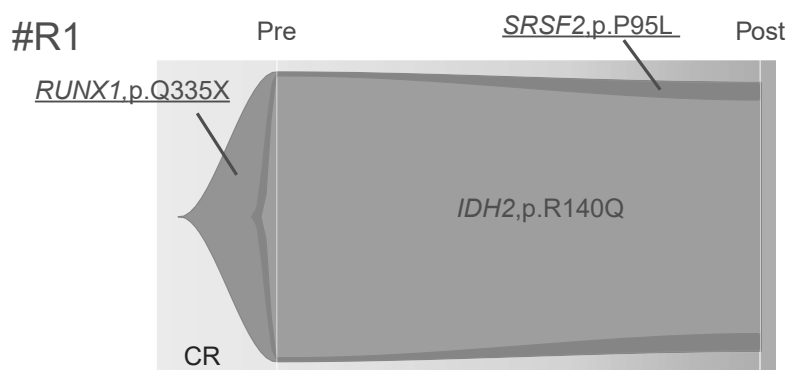

\#R5

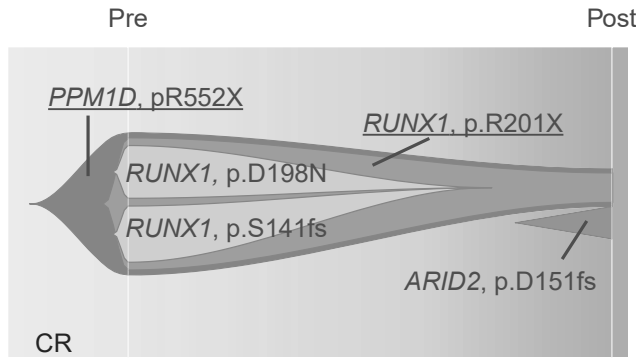

\#R2
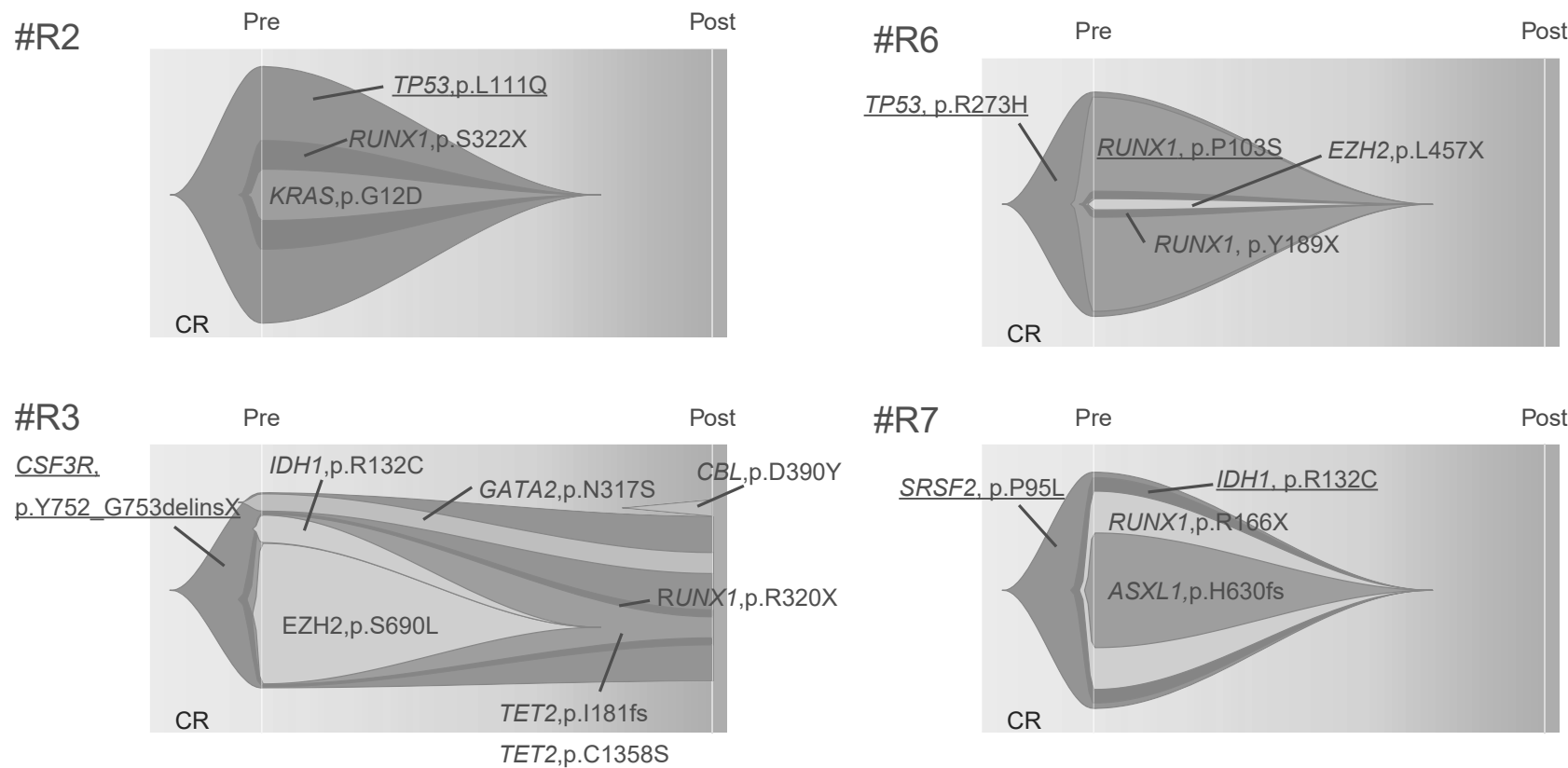

\#R4

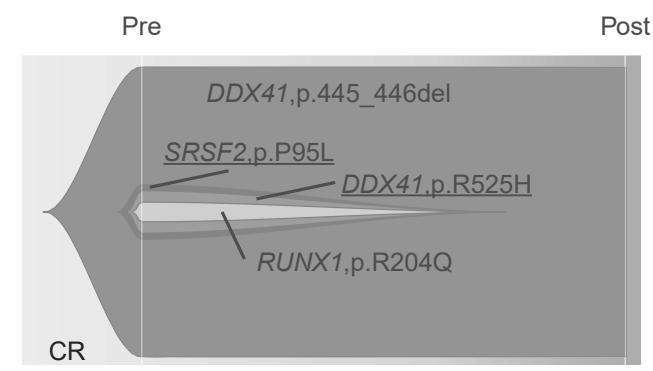

\#R8

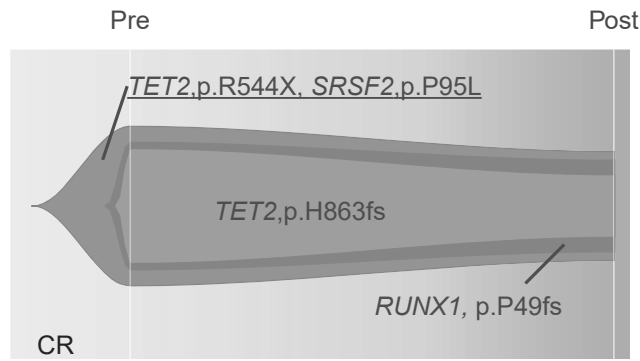

\section{Supplementary Fig. 5 | Fish plots of CR cases harboring mutations in RUNX1.}

Fishplots showing changes in clonal structures assumed from variant allele frequency (VAF) values including mutations in RUNX1 gene in pre-treatment samples that achieved complete remission (CR). Clones marked with underline represent major clones (MC). All the 11 clones represented by RUNX1 gene mutations reduced their sizes. Three of these clones were MC (\#R1:RUNX1,p.Q335X, \#R5:RUNX1, p.R201X, \#R6:RUNX1, p.P103S) and the other 8 clones were non-MC and were assumed to be subclones. Six out of of these non-MC (\#R2:RUNX1,p.S322X, \#R4:RUNX1,p.R204Q, \#R5:RUNX1, p.D198N, RUNX1, p.S141fs, \#R6:RUNX1, p.Y189X, \#R7:RUNX1,p.R166X) were included by parent clones that also reduced their sizes. 


\section{Supplementary Files}

This is a list of supplementary files associated with this preprint. Click to download.

- SupplementaryTables.xlsx

- DataTable.xIsx 